\title{
Enantioselective alkylation of amino acid derivatives promoted by cyclic peptoids under phase-transfer conditions
}

Rosaria Schettini, Francesco De Riccardis, Giorgio Della Sala,* and Irene Izzo*

\section{Supporting Information}

\section{Contents}

1. Screening of the catalysts $\mathbf{3}$ and $\mathbf{4}$ in the phase transfer benzylation of tert-butyl ester $\mathbf{1 a}$

2. Optimization of the catalyst's loading in the phase transfer alkylation of $\mathbf{1 d}$

3. Computational details

S4-S5

4. References

5. HPLC chromatograms and ${ }^{1} \mathrm{H}$ NMR and ${ }^{13} \mathrm{C}$ NMR spectra of peptoids $\mathbf{3 a - 3 e}, \mathbf{4 a - 4 i}$ and $\mathbf{7}$ S7-S21

6. 2D NMR spectra of peptoid $\mathbf{3 b}$

S22-S24

7. ${ }^{1} \mathrm{H}$ NMR and ${ }^{13} \mathrm{C}$ NMR spectra of compounds $\mathbf{2 d a - 2 d o}$

S25-S39

8. Chiral HPLC traces of compounds 2da-2do

S40-S54

* Corresponding author. Tel.: +39-089-969560; fax: +39-089-969603; e-mail: iizzo@unisa.it 


\section{Screening of the catalysts 3 and 4 in the phase transfer benzylation of tert-}

butyl ester 1a. ${ }^{a}$
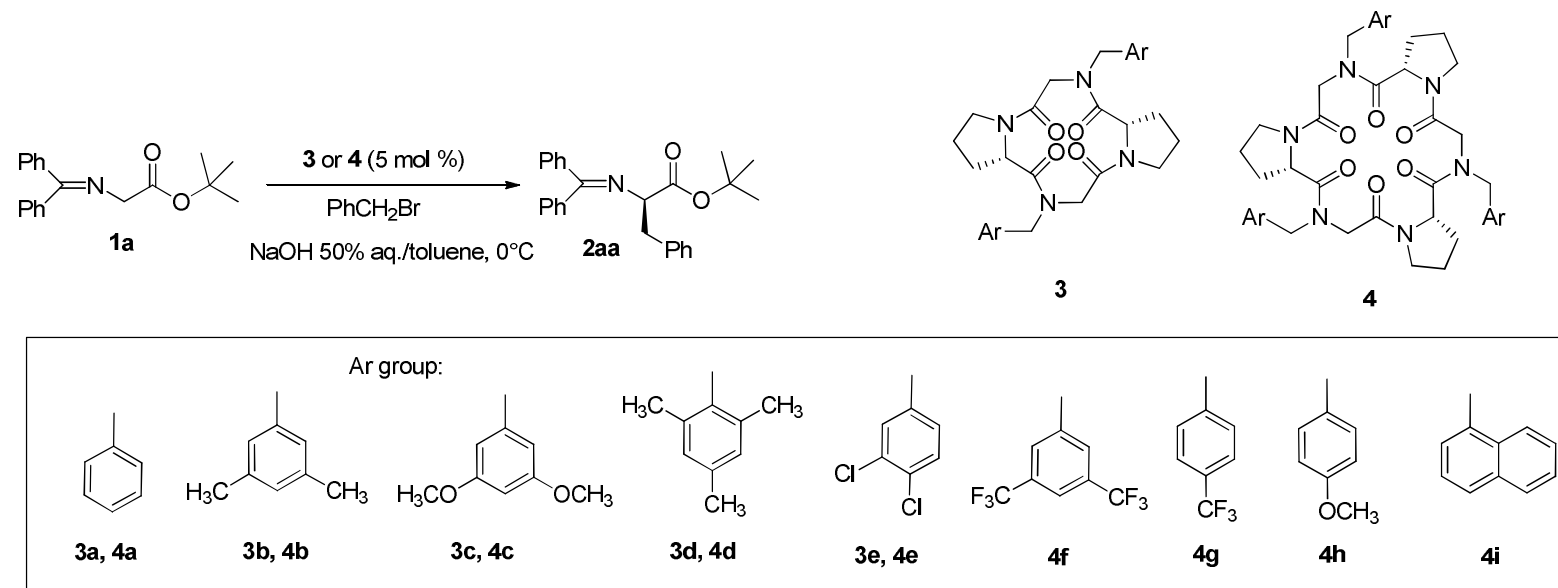

$4 \mathbf{4}$

\begin{tabular}{cccc}
\hline entry & catalyst & yield $(\%)^{b}$ & ee $(\%)^{c}$ \\
\hline 1 & $\mathbf{4 a}$ & 91 & 75 \\
3 & $\mathbf{4 b}$ & 64 & 67 \\
4 & $\mathbf{4 c}$ & 68 & 64 \\
5 & $\mathbf{4 d}$ & 80 & 25 \\
6 & $\mathbf{4 e}$ & 68 & 13 \\
7 & $\mathbf{4 f}$ & 65 & 28 \\
8 & $\mathbf{4 g}$ & 7 & 30 \\
9 & $\mathbf{4 h}$ & 53 & 48 \\
10 & $\mathbf{4 i}$ & 77 & 61 \\
11 & $\mathbf{3 a}$ & 67 & 36 \\
12 & $\mathbf{3 b}$ & 51 & 41 \\
13 & $\mathbf{3 c}$ & 64 & 10 \\
14 & $\mathbf{3 d}$ & 75 & 39 \\
\hline
\end{tabular}

${ }^{a}$ All reactions were performed in a liquid-liquid system with $0.08 \mathrm{mmol}$ of $\mathbf{1 a}$, benzyl bromide (1.2 equiv.), and catalyst $(5 \mathrm{~mol} \%)$ in toluene $(0.8 \mathrm{~mL})$ and $\mathrm{NaOH} 50 \%$ aq. ${ }^{b}$ Isolated yields. ${ }^{c}$ Determined by HPLC using a Chiralcel OD-H chiral stationary phase. ${ }^{d}$ The $(S)$ alkylation products was obtained as major enantiomer. 
2. Optimization of the catalyst's loading in the phase transfer alkylation of $1 \mathrm{~d}^{a}$

\begin{tabular}{|c|c|c|c|c|c|}
\hline entry & $\mathrm{RBr}$ & product & amount of catalyst & yield $(\%)^{b}$ & ee $(\%)^{c}$ \\
\hline 1 & & & $2.5 \% \mathrm{~mol}$ & 75 & 93 \\
\hline 2 & & 2da & $1.0 \% \mathrm{~mol}$ & 76 & 92 \\
\hline 3 & & & no catalyst & 22 & - \\
\hline 4 & & & $2.5 \% \mathrm{~mol}$ & 73 & 84 \\
\hline 5 & & 200 & $1.0 \% \mathrm{~mol}$ & 75 & 94 \\
\hline 6 & & & $2.5 \% \mathrm{~mol}$ & 75 & 72 \\
\hline 7 & & 2dc & $1.0 \% \mathrm{~mol}$ & 82 & 89 \\
\hline 8 & & & $2.5 \% \mathrm{~mol}$ & 89 & 96 \\
\hline 9 & & 200 & $1.0 \% \mathrm{~mol}$ & 84 & 92 \\
\hline 10 & & & $2.5 \% \mathrm{~mol}$ & 74 & 88 \\
\hline 11 & & . & $1.0 \% \mathrm{~mol}$ & 53 & 76 \\
\hline 12 & & & $2.5 \% \mathrm{~mol}$ & 89 & 89 \\
\hline 13 & & 201 & $1.0 \% \mathrm{~mol}$ & 75 & 79 \\
\hline 14 & & & $2.5 \% \mathrm{~mol}$ & 72 & 81 \\
\hline 15 & & $20 \mathrm{~g}$ & $1.0 \% \mathrm{~mol}$ & 80 & 93 \\
\hline 16 & & & $2.5 \% \mathrm{~mol}$ & 75 & 91 \\
\hline 17 & & 2011 & $1.0 \% \mathrm{~mol}$ & 74 & 85 \\
\hline 18 & & & $2.5 \% \mathrm{~mol}$ & 86 & 93 \\
\hline 19 & & & $1.0 \% \mathrm{~mol}$ & 87 & 94 \\
\hline 20 & & & $2.5 \% \mathrm{~mol}$ & 97 & 95 \\
\hline 21 & & $2 \mathrm{uJ}$ & $1.0 \% \mathrm{~mol}$ & 83 & 92 \\
\hline 21 & & & $2.5 \% \mathrm{~mol}$ & 90 & 93 \\
\hline 22 & & $2 \mathbf{a k}$ & $1.0 \% \mathrm{~mol}$ & 87 & 70 \\
\hline
\end{tabular}




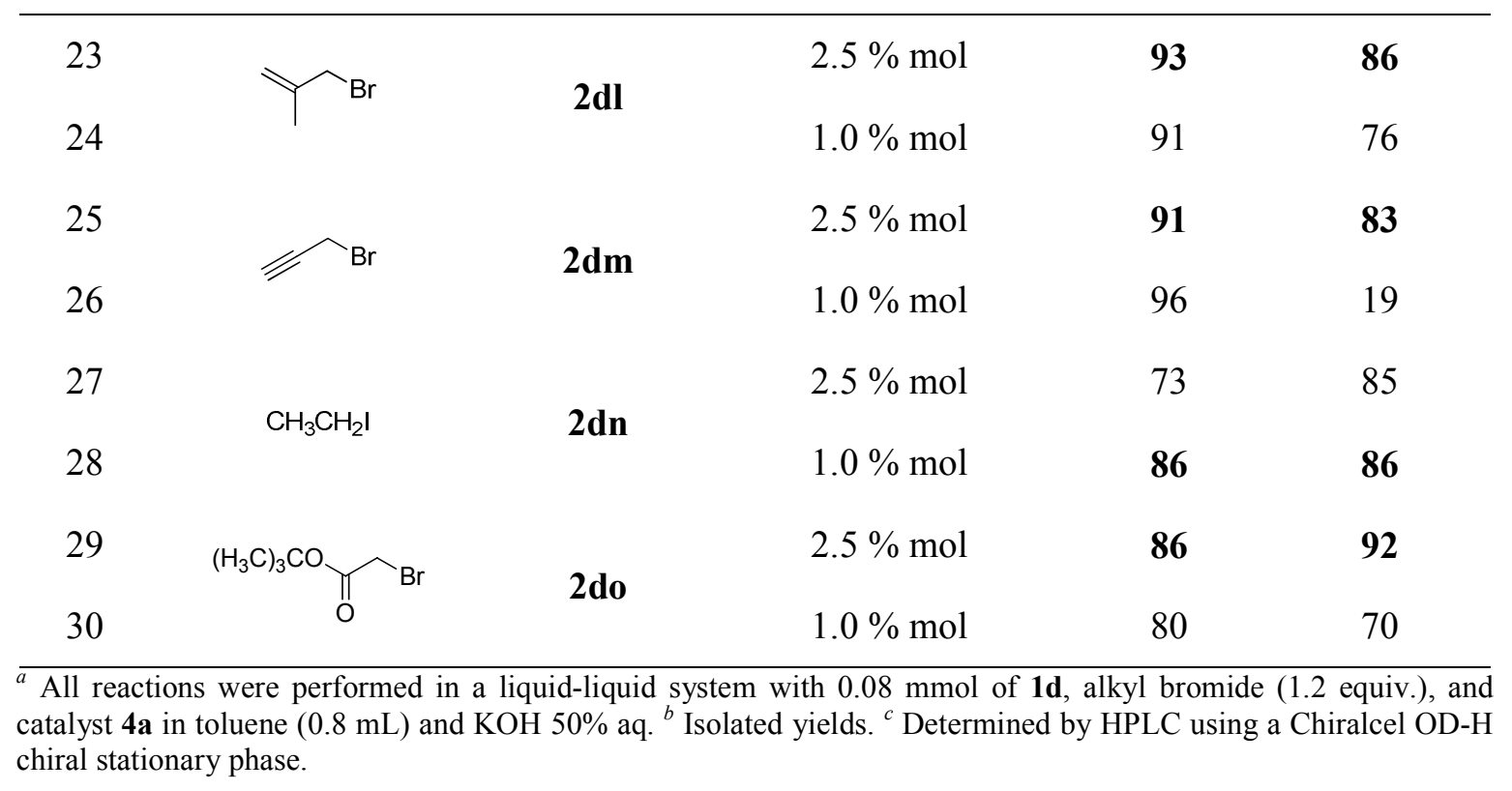

\section{Computational details}

Density functional calculations were performed on all the systems with the Gaussian09 set of programs. ${ }^{1}$ BP86 was used as a functional and gradient corrections were taken from the works of Becke $^{2}$ and Perdew. ${ }^{3}$ The electronic configuration of the molecular systems was described by the split-valence basis set with polarization functions of Ahlirchs and co-worker (standard SVP basis set in Gaussian09), for H, C, N, and O. ${ }^{4}$ Minimum free-energy structures were characterized by the presence of zero imaginary frequency.

\subsection{Cartesian coordinates of the cyclopeptoid $3 \boldsymbol{b}$ structure}

80

$\begin{array}{rrrr}\mathrm{O} & 2.673041 & -3.187140 & -1.264518 \\ \mathrm{O} & -0.867981 & -1.805740 & 0.718647 \\ \mathrm{O} & -2.672681 & 3.187240 & -1.263528 \\ \mathrm{O} & 0.867747 & 1.805730 & 0.718424 \\ \mathrm{~N} & 1.749164 & -0.057603 & -0.286051 \\ \mathrm{~N} & -0.478008 & 2.652486 & -1.525094 \\ \mathrm{~N} & -1.749160 & 0.057660 & -0.285887 \\ \mathrm{~N} & 0.478201 & -2.652385 & -1.524640 \\ \mathrm{C} & 1.801574 & -2.321720 & -1.399981 \\ \mathrm{C} & 2.184235 & -0.821100 & -1.455439 \\ \mathrm{H} & 3.293316 & -0.814996 & -1.526116 \\ \mathrm{H} & 1.792887 & -0.360053 & -2.381564 \\ \mathrm{C} & 2.242416 & -0.550781 & 1.022940 \\ \mathrm{H} & 2.039131 & -1.639099 & 1.072477 \\ \mathrm{H} & 1.625493 & -0.049859 & 1.790689 \\ \mathrm{C} & 3.718556 & -0.262636 & 1.243666 \\ \mathrm{C} & 4.694440 & -1.222245 & 0.913595 \\ \mathrm{H} & 4.375995 & -2.192650 & 0.494848 \\ \mathrm{C} & 6.070856 & -0.952661 & 1.095924 \\ \mathrm{C} & 6.444252 & 0.302245 & 1.613299\end{array}$




\begin{tabular}{|c|c|c|c|}
\hline $\mathrm{H}$ & 7.516065 & 0.523492 & 1.759596 \\
\hline $\mathrm{C}$ & 5.487094 & 1.286014 & 1.953657 \\
\hline $\mathrm{C}$ & 4.124972 & 0.988464 & 1.760896 \\
\hline $\mathrm{H}$ & 3.355886 & 1.735741 & 2.017726 \\
\hline $\mathrm{C}$ & 7.106348 & -2.002462 & 0.755116 \\
\hline $\mathrm{H}$ & 8.135018 & -1.590589 & 0.786188 \\
\hline $\mathrm{H}$ & 6.935458 & -2.428628 & -0.255739 \\
\hline $\mathrm{H}$ & 7.064356 & -2.853346 & 1.469653 \\
\hline $\mathrm{C}$ & 5.926253 & 2.617731 & 2.524042 \\
\hline $\mathrm{H}$ & 5.069081 & 3.307279 & 2.657529 \\
\hline $\mathrm{H}$ & 6.668012 & 3.118206 & 1.865430 \\
\hline $\mathrm{H}$ & 6.413459 & 2.491997 & 3.515391 \\
\hline C & 1.090544 & 1.154860 & -0.307138 \\
\hline C & 0.658645 & 1.742757 & -1.681917 \\
\hline $\mathrm{H}$ & 0.404089 & 0.948761 & -2.411196 \\
\hline C & 1.764143 & 2.682220 & -2.236305 \\
\hline $\mathrm{H}$ & 1.637058 & 2.786418 & -3.335291 \\
\hline $\mathrm{H}$ & 2.786414 & 2.297018 & -2.047012 \\
\hline C & 1.468793 & 4.013290 & -1.517683 \\
\hline $\mathrm{H}$ & 1.882314 & 3.983343 & -0.490240 \\
\hline $\mathrm{H}$ & 1.905231 & 4.887447 & -2.040499 \\
\hline C & -0.067735 & 4.068340 & -1.459559 \\
\hline $\mathrm{H}$ & -0.458471 & 4.537709 & -0.534059 \\
\hline $\mathrm{H}$ & -0.513236 & 4.620629 & -2.316957 \\
\hline C & -1.801318 & 2.321826 & -1.399685 \\
\hline C & -2.184044 & 0.821227 & -1.455314 \\
\hline $\mathrm{H}$ & -3.293118 & 0.815155 & -1.526125 \\
\hline $\mathrm{H}$ & -1.792566 & 0.360192 & -2.381387 \\
\hline C & -2.242587 & 0.550779 & 1.023066 \\
\hline $\mathrm{H}$ & -2.039387 & 1.639105 & 1.072636 \\
\hline $\mathrm{H}$ & -1.625720 & 0.049874 & 1.790870 \\
\hline C & -3.718740 & 0.262575 & 1.243616 \\
\hline C & -4.694588 & 1.222223 & 0.913528 \\
\hline $\mathrm{H}$ & -4.376068 & 2.192648 & 0.494894 \\
\hline C & -6.071018 & 0.952645 & 1.095722 \\
\hline $\mathrm{C}$ & -6.444472 & -0.302300 & 1.612969 \\
\hline $\mathrm{H}$ & -7.516300 & -0.523539 & 1.759166 \\
\hline C & -5.487357 & -1.286108 & 1.953322 \\
\hline C & -4.125210 & -0.988561 & 1.760711 \\
\hline $\mathrm{H}$ & -3.356153 & -1.735859 & 2.017556 \\
\hline C & -7.106476 & 2.002467 & 0.754888 \\
\hline $\mathrm{H}$ & -6.935740 & 2.428407 & -0.256097 \\
\hline $\mathrm{H}$ & -7.064269 & 2.853508 & 1.469225 \\
\hline $\mathrm{H}$ & -8.135180 & 1.590698 & 0.786225 \\
\hline C & -5.926588 & -2.617882 & 2.523525 \\
\hline $\mathrm{H}$ & -5.069393 & -3.307339 & 2.657318 \\
\hline $\mathrm{H}$ & -6.668040 & -3.118424 & 1.864615 \\
\hline $\mathrm{H}$ & -6.414194 & -2.492222 & 3.514687 \\
\hline C & -1.090589 & -1.154822 & -0.306933 \\
\hline $\mathrm{C}$ & -0.658496 & -1.742727 & -1.681641 \\
\hline $\mathrm{H}$ & -0.403912 & -0.948759 & -2.410937 \\
\hline C & -1.763883 & -2.682275 & -2.236129 \\
\hline $\mathrm{H}$ & -2.786197 & -2.297148 & -2.046906 \\
\hline $\mathrm{H}$ & -1.636706 & -2.786444 & -3.335107 \\
\hline C & -1.468482 & -4.013334 & -1.517516 \\
\hline $\mathrm{H}$ & -1.904851 & -4.887519 & -2.040341 \\
\hline $\mathrm{H}$ & -1.882015 & -3.983404 & -0.490078 \\
\hline C & 0.068042 & -4.068281 & -1.459371 \\
\hline $\mathrm{H}$ & 0.458785 & -4.537795 & -0.533946 \\
\hline $\mathrm{H}$ & 0.513611 & -4.620379 & -2.316856 \\
\hline
\end{tabular}




\section{References}

(1) Frisch, M. J.; Trucks, G. W.; Schlegel, H. B.; Scuseria, G. E.; Robb, M. A.; Cheeseman, J. R.; Scalmani, G.; Barone, V.; Mennucci, B.; Petersson, G. A.; Nakatsuji, H.; Caricato, M.; Li, X.; Hratchian, H. P.; Izmaylov, A. F.; Bloino, J.; Zheng, G.; Sonnenberg, J. L.; Hada, M.; Ehara, M.; Toyota, K.; Fukuda, R.; Hasegawa, J.; Ishida, M.; Nakajima, T.; Honda, Y.; Kitao, O.; Nakai, H.; Vreven, T.; Montgomery Jr., J. A.; Peralta, J. E.; Ogliaro, F.; Bearpark, M.; Heyd, J. J.; Brothers, E. N.; Kudin, K.; Staroverov, V. N.; Kobayashi, R.; Normand, J.; Raghavachari, K.; Rendell, A.; Burant, J. C.; Iyengar, S. S.; Tomasi, J.; Cossi, M.; Rega, N.; Millam, J. M.; Klene, M.; Knox, J. E.; Cross, J. B.; Bakken, V.; Adamo, C.; Jaramillo, J.; Gomperts, R.; Stratmann, R. E.; Yazyev, O.; Austin, A. J.; Cammi, R.; Pomelli, C.; Ochterski, J. W.; Martin, R. L.; Morokuma, K.; Zakrzewski, V. G.; Voth, G. A.; Salvador, P.; Dannenberg, J. J.; Dapprich, S.; Daniels, A. D.; Farkas, Ö; Foresman, J. B.; Ortiz, J. V.; Cioslowski, J.; Fox, D. J. Gaussian 09, Revision A.02, Gaussian, Inc., Wallingford, CT, 2009.

(2) Becke, A. Phys. Rev. A 1988, 38, 3098-3100.

(3) Perdew, J. P. Phys. Rev. B 1986, 33, 8822-8824.

(4) Schaefer, A.; Horn, H.; Ahlrichs, R. J. Chem. Phys. 1992, 97, 2571-2577. 


\section{HPLC chromatograms and 1H NMR and 13C NMR spectra of peptoid 3a-3e,}

\section{4a-4i and 7}

RP-HPLC of compound 3a

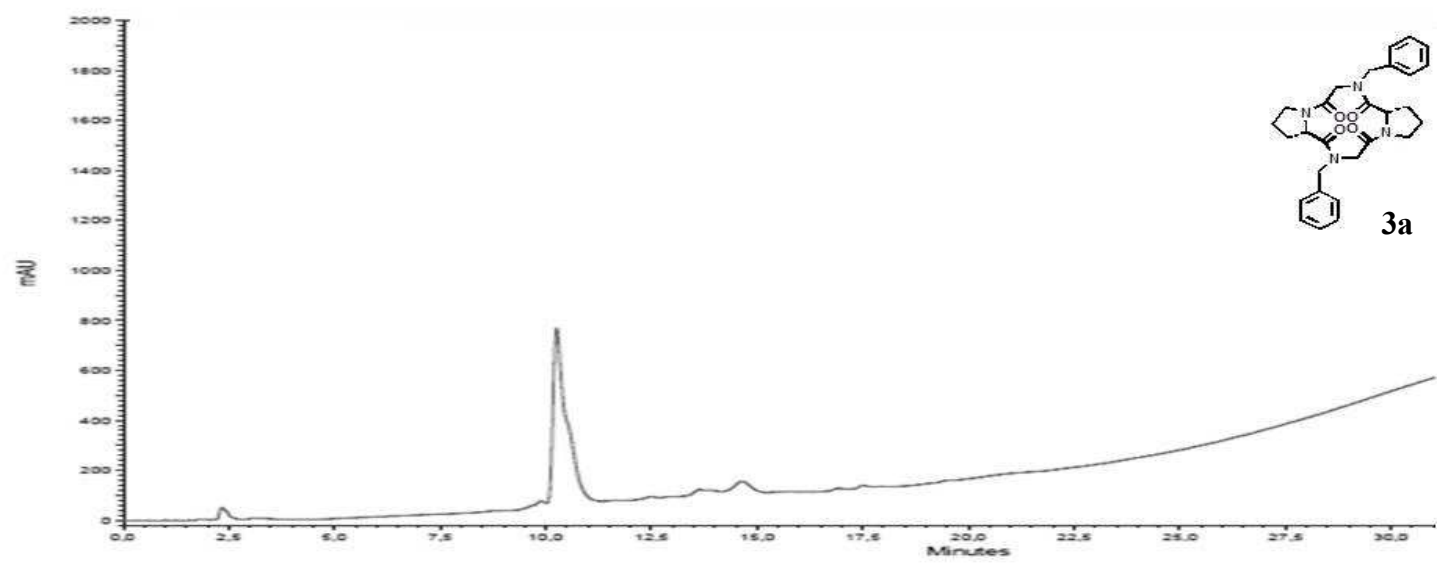

${ }^{1} \mathrm{H}$ NMR of compound $\mathbf{3 a}\left(400 \mathrm{MHz}, \mathrm{CDCl}_{3}\right)$

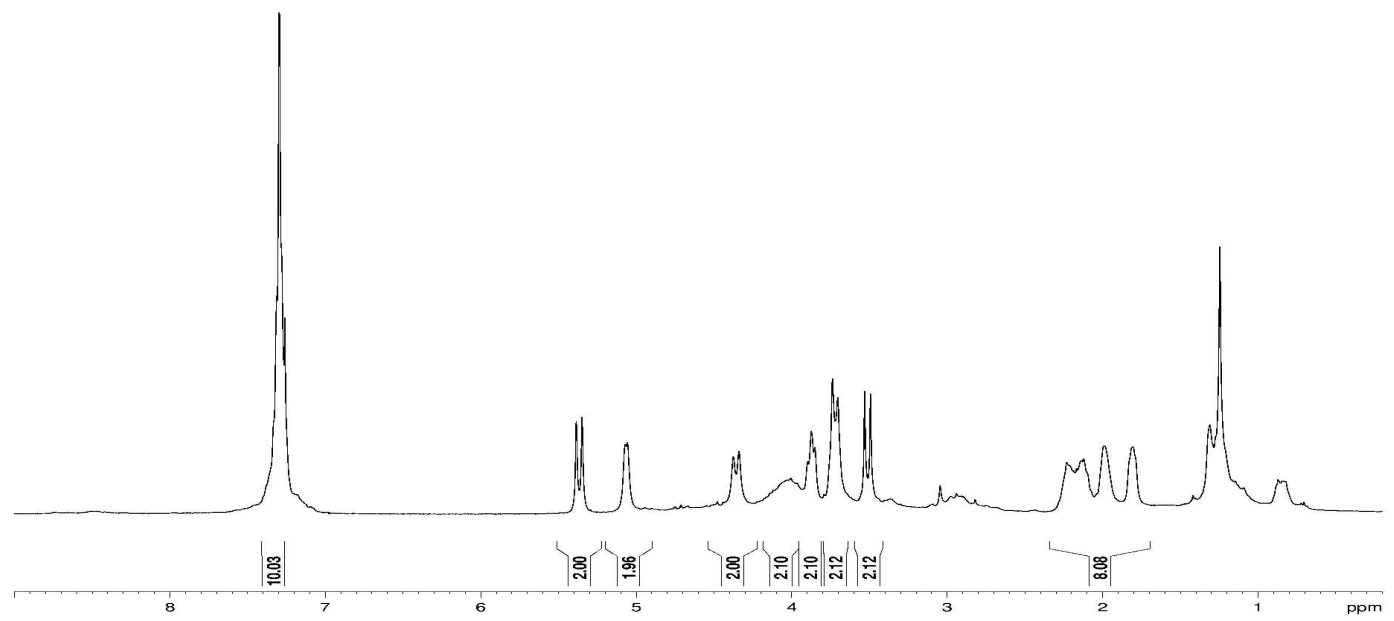

${ }^{13} \mathrm{C}$ NMR of compound 3a $\left(100 \mathrm{MHz}, \mathrm{CDCl}_{3}\right)$

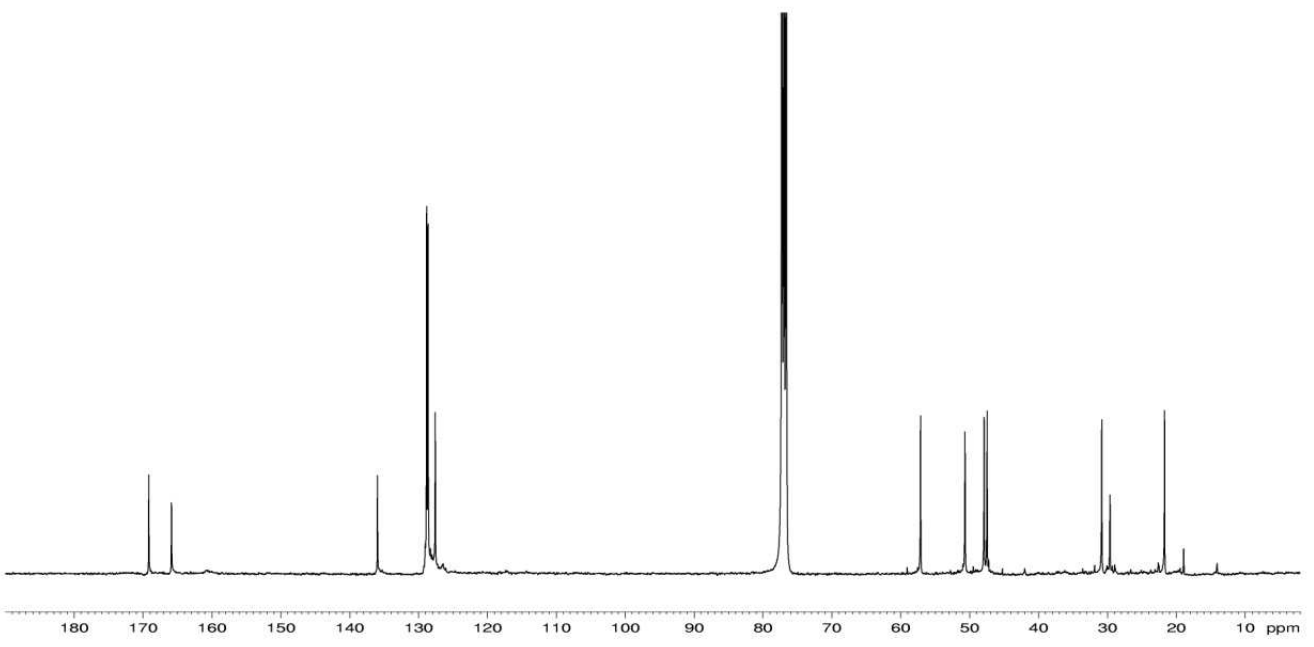




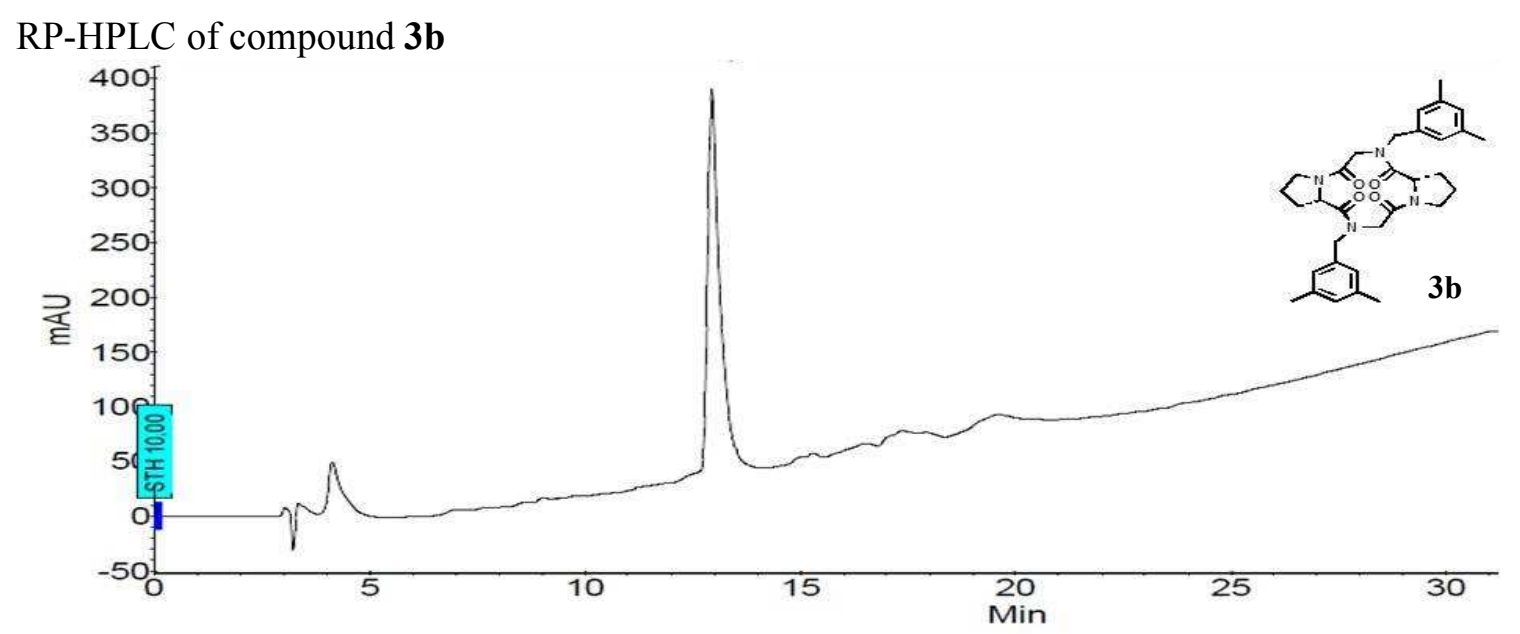

${ }^{1} \mathrm{H}$ NMR of compound $\mathbf{3 b}\left(600 \mathrm{MHz}, \mathrm{DMSO}-d_{6}\right)$

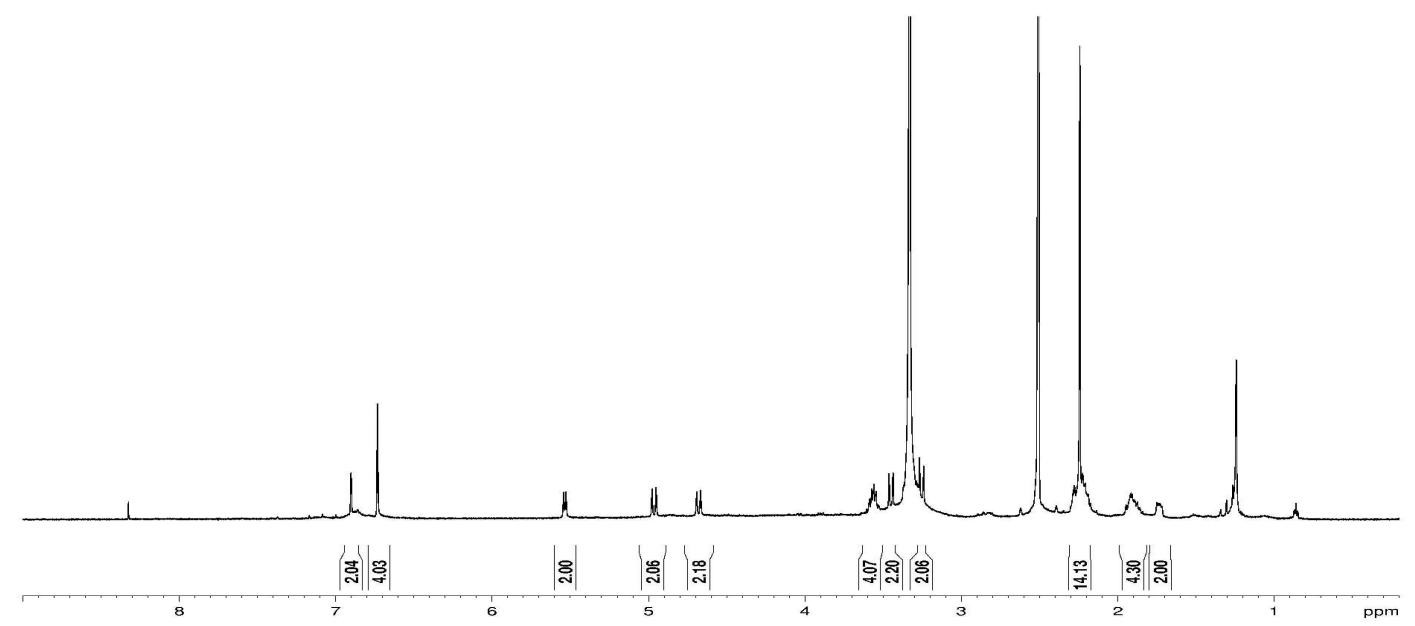

${ }^{13} \mathrm{C}$ NMR of compound $\mathbf{3 b}\left(150 \mathrm{MHz}\right.$, DMSO- $\left.d_{6}\right)$

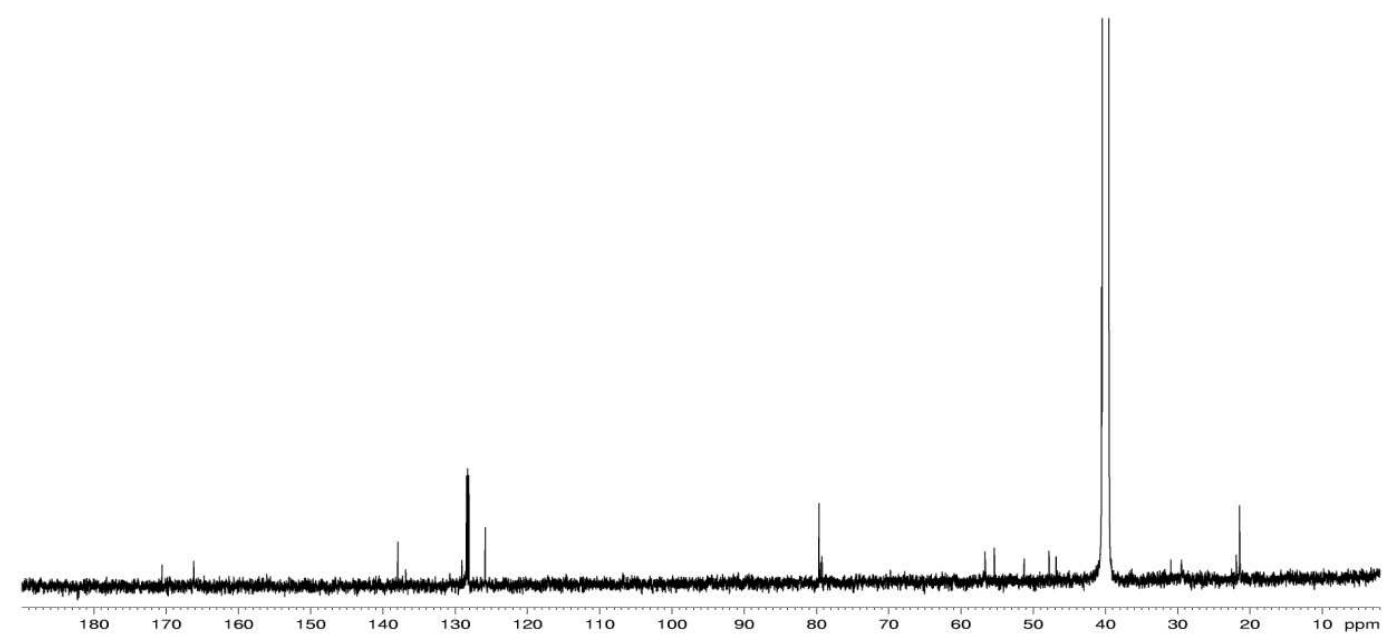


RP-HPLC of compound 3c

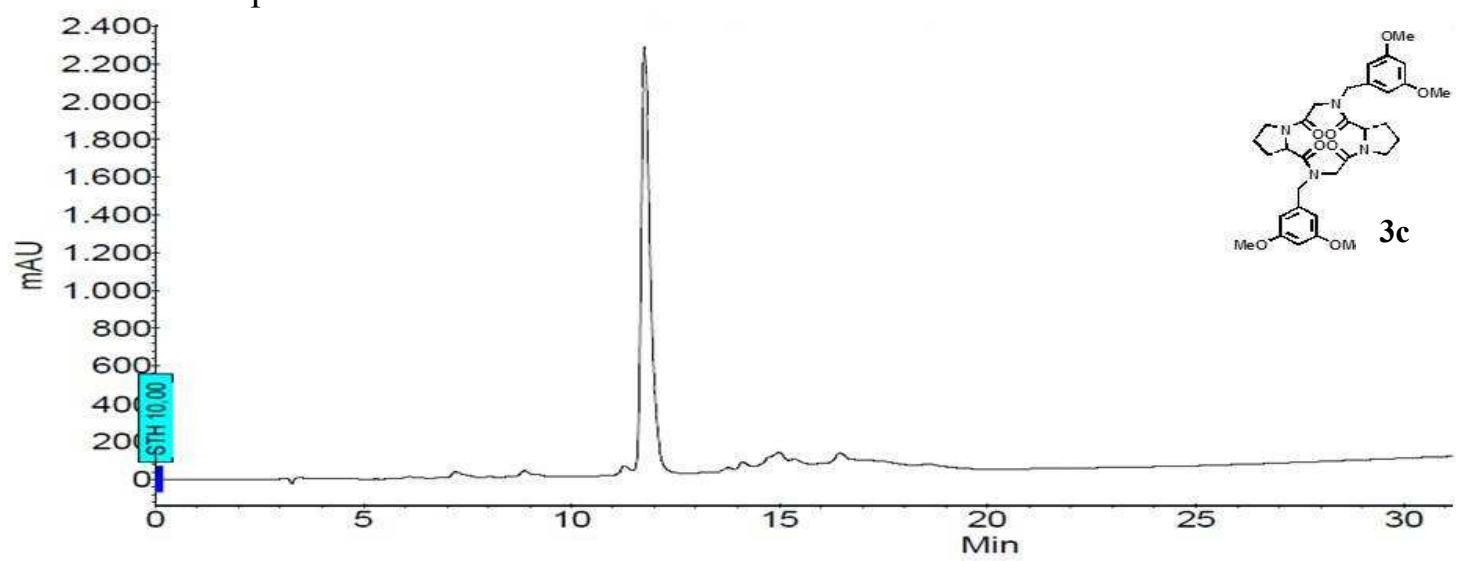

${ }^{1} \mathrm{H}$ NMR of compound $\mathbf{3 c}\left(300 \mathrm{MHz}, \mathrm{CDCl}_{3}\right)$

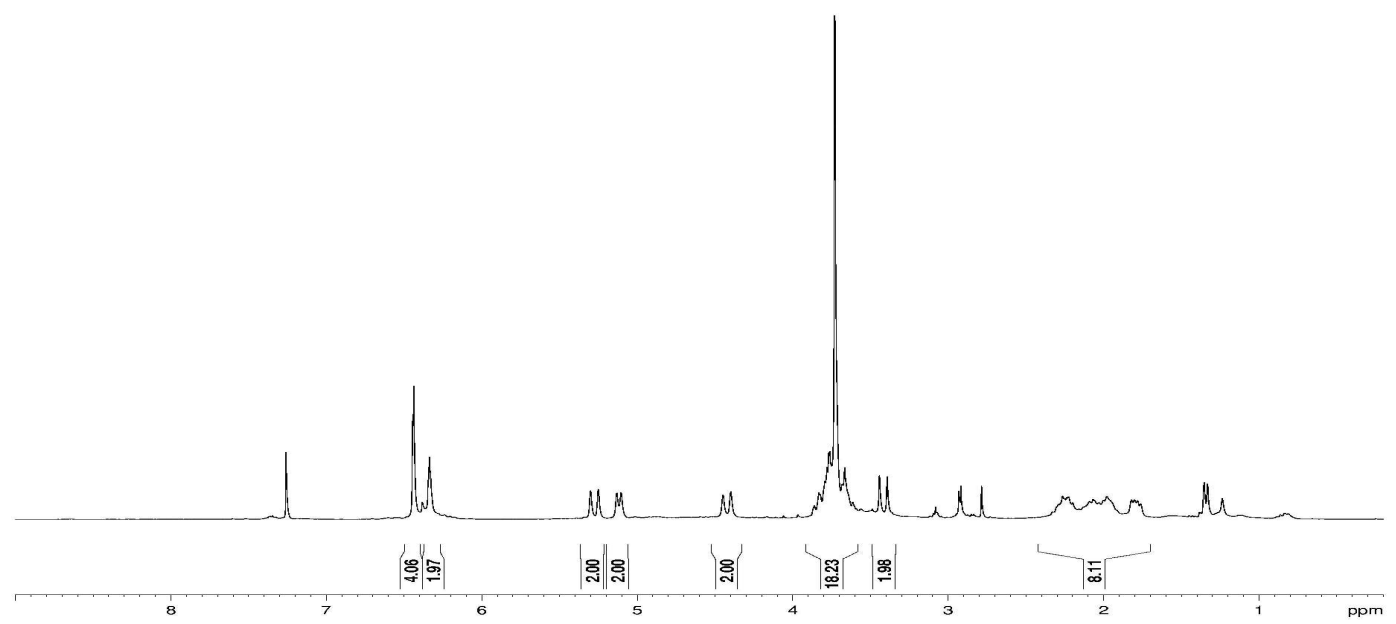

${ }^{13} \mathrm{C}$ NMR of compound $\mathbf{3 c}\left(75 \mathrm{MHz}, \mathrm{CDCl}_{3}\right)$

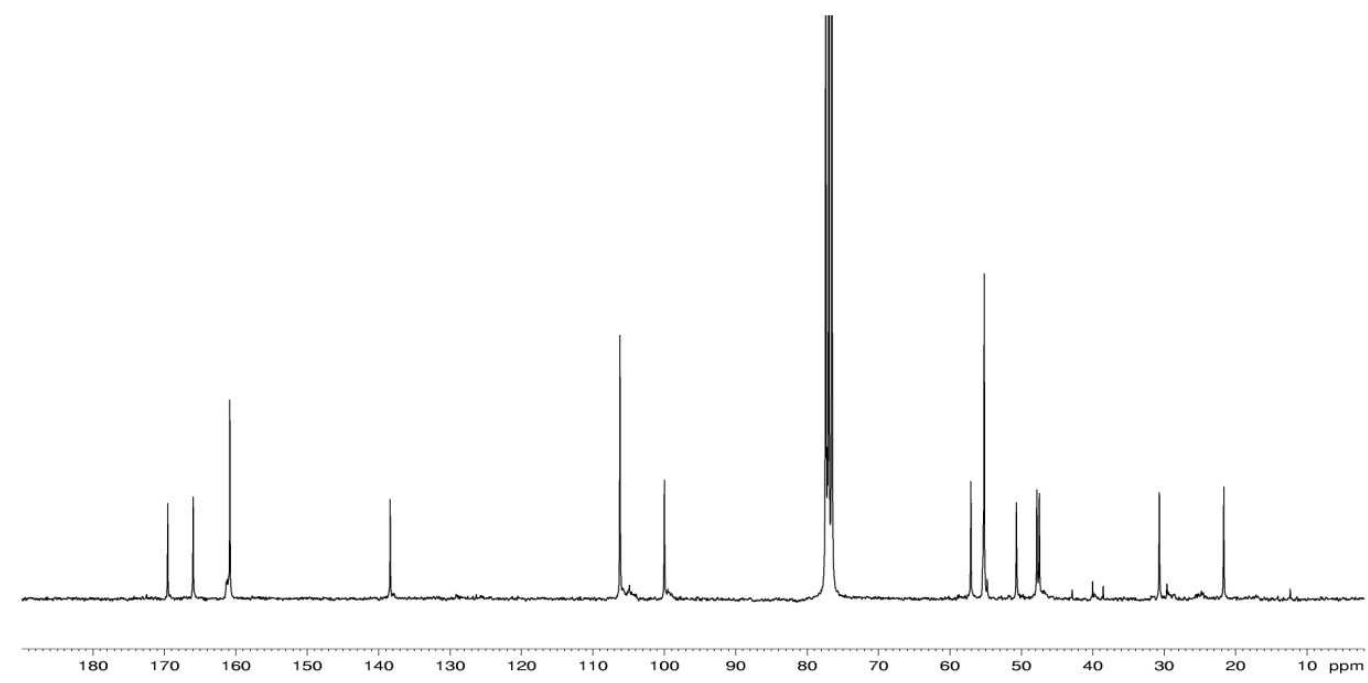


RP-HPLC of compound 3d

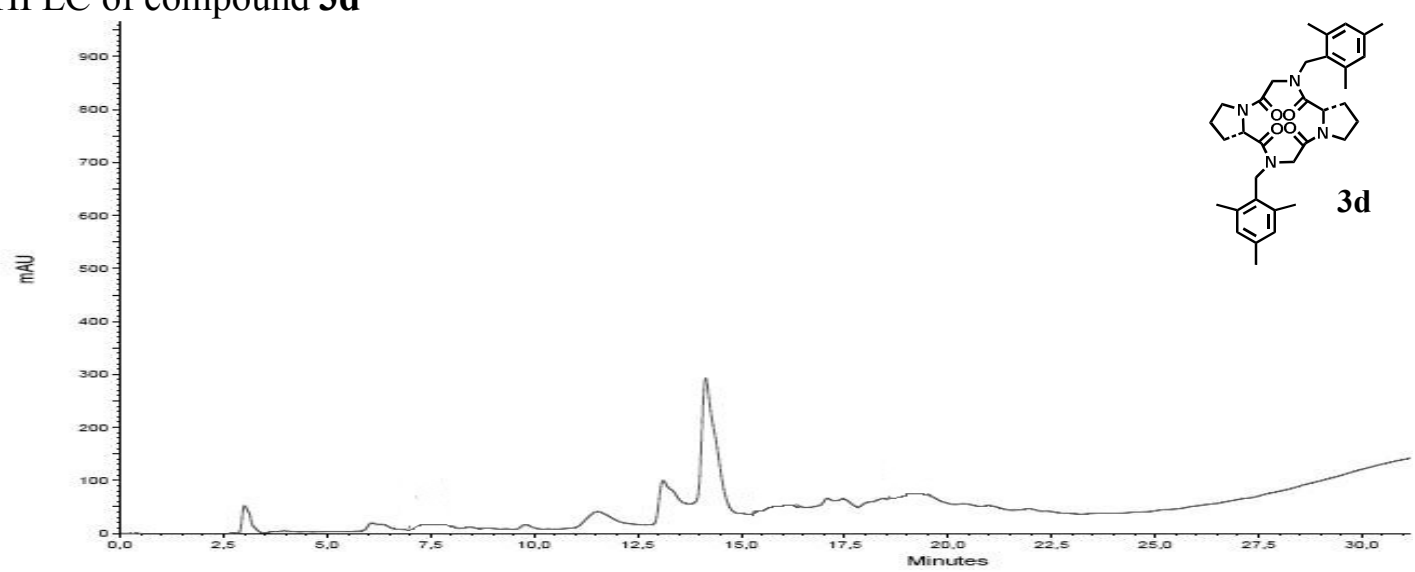

${ }^{1} \mathrm{H}$ NMR of compound $3 \mathbf{d}\left(600 \mathrm{MHz}, \mathrm{CDCl}_{3}\right)$

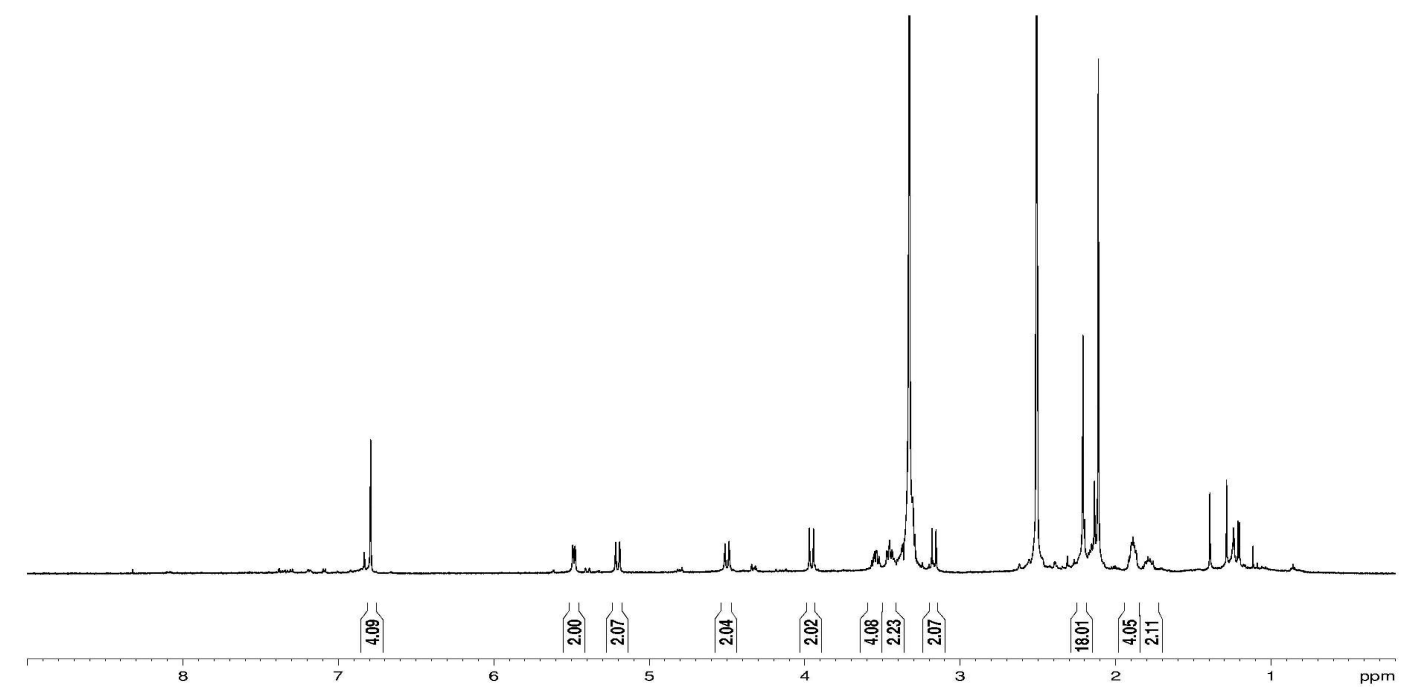

${ }^{13} \mathrm{C}$ NMR of compound 3d $\left(100 \mathrm{MHz}, \mathrm{CDCl}_{3}\right)$

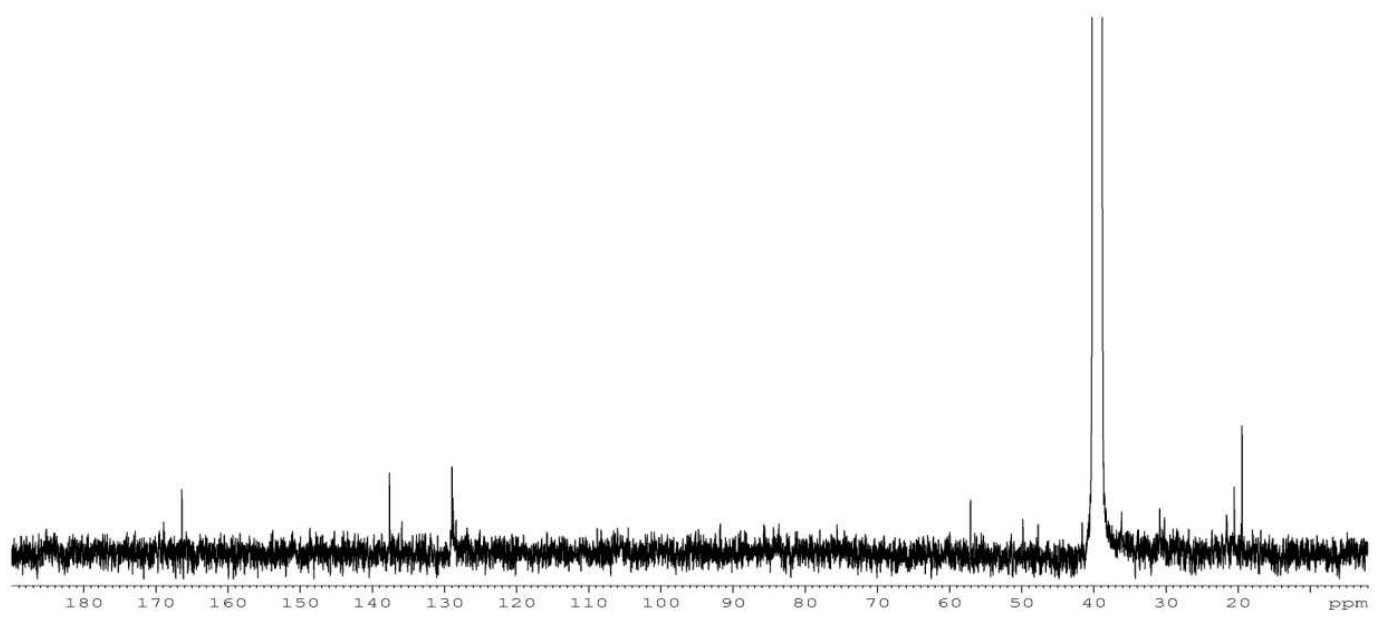




\section{RP-HPLC of compound $\mathbf{3 e}$}

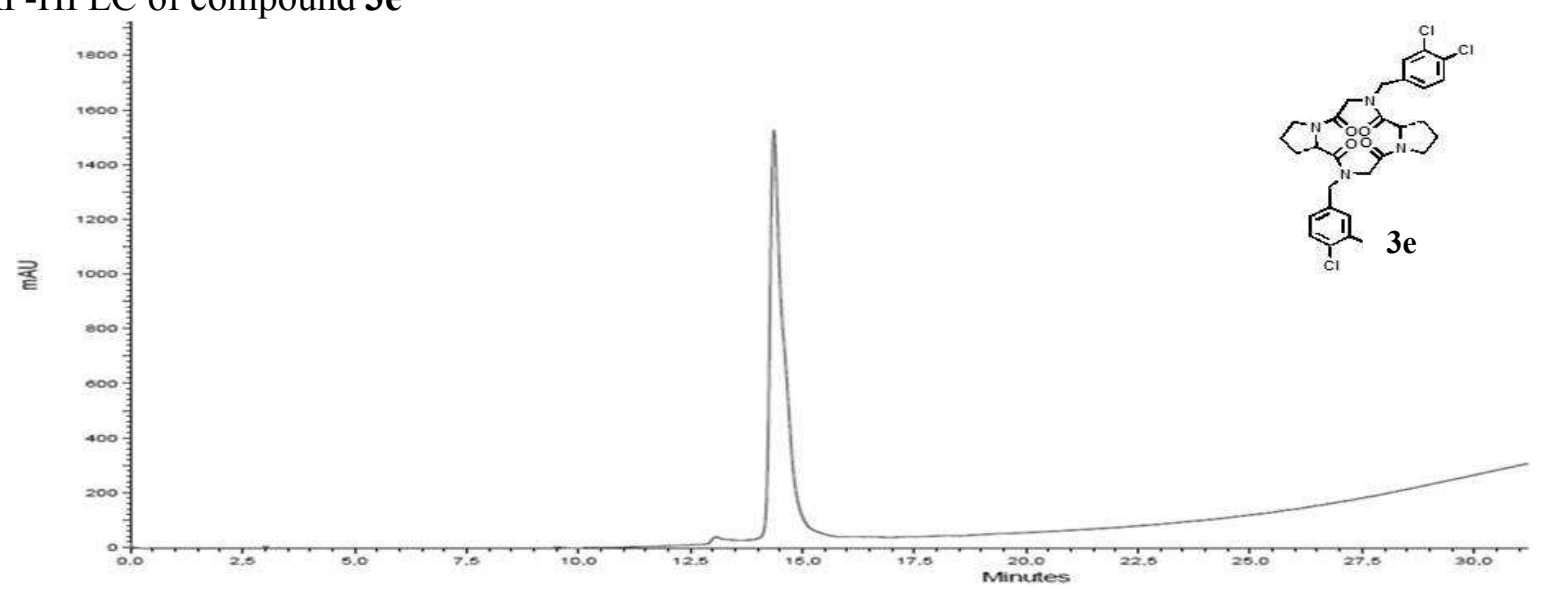

${ }^{1} \mathrm{H}$ NMR of compound $\mathbf{3 e}\left(400 \mathrm{MHz}, \mathrm{CDCl}_{3}\right)$

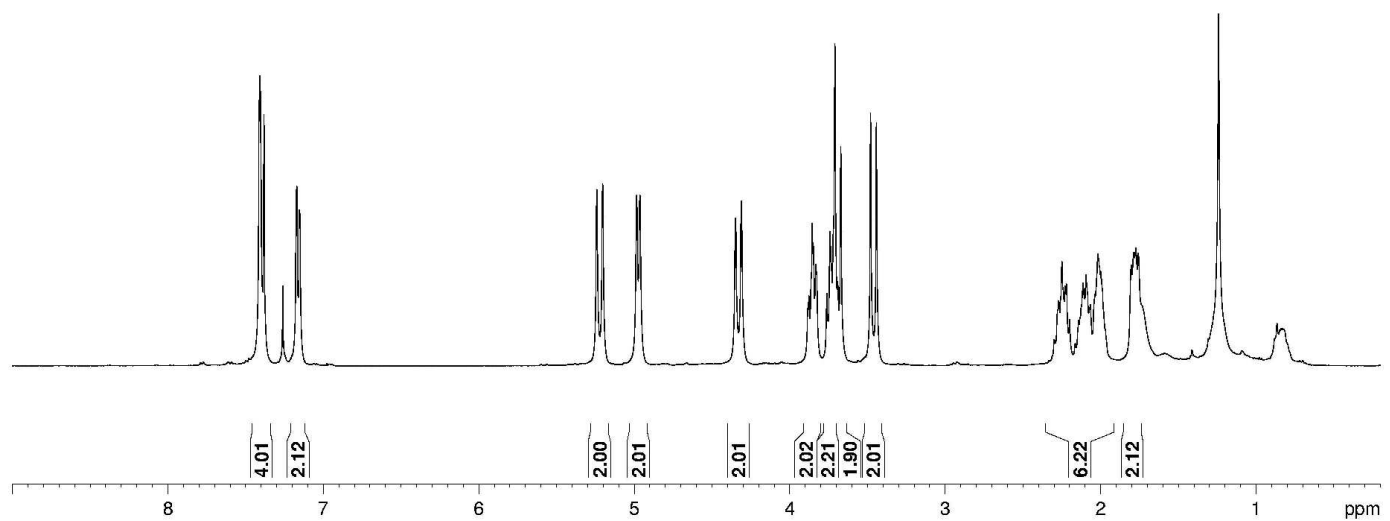

${ }^{13} \mathrm{C}$ NMR of compound $\mathbf{3 e}\left(100 \mathrm{MHz}, \mathrm{CDCl}_{3}\right)$

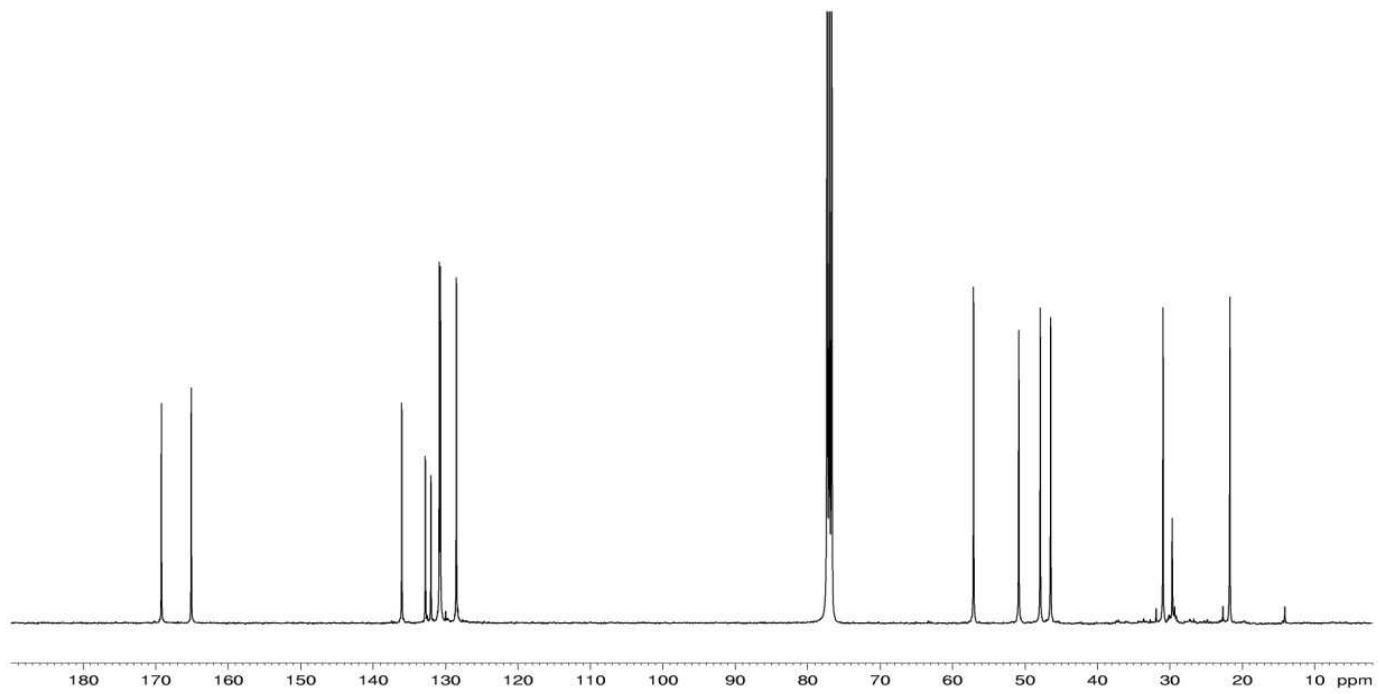


RP-HPLC of compound $\mathbf{4 a}$

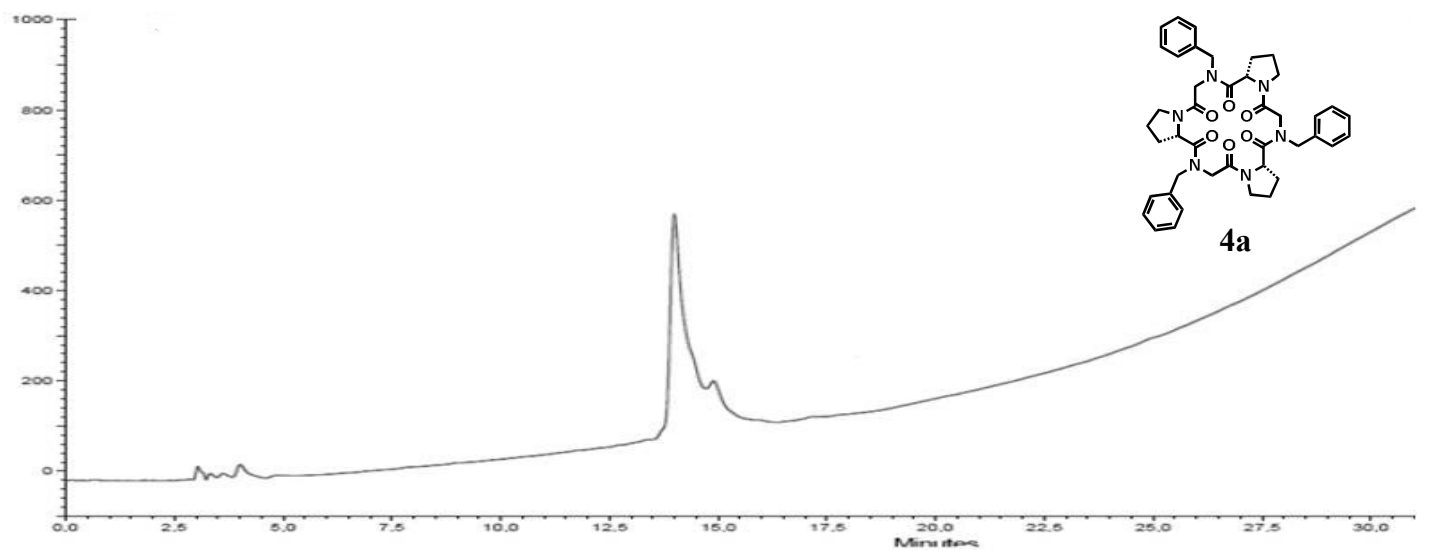

${ }^{1} \mathrm{H}$ NMR of compound $\mathbf{4 a}\left(400 \mathrm{MHz}, \mathrm{CDCl}_{3}\right)$

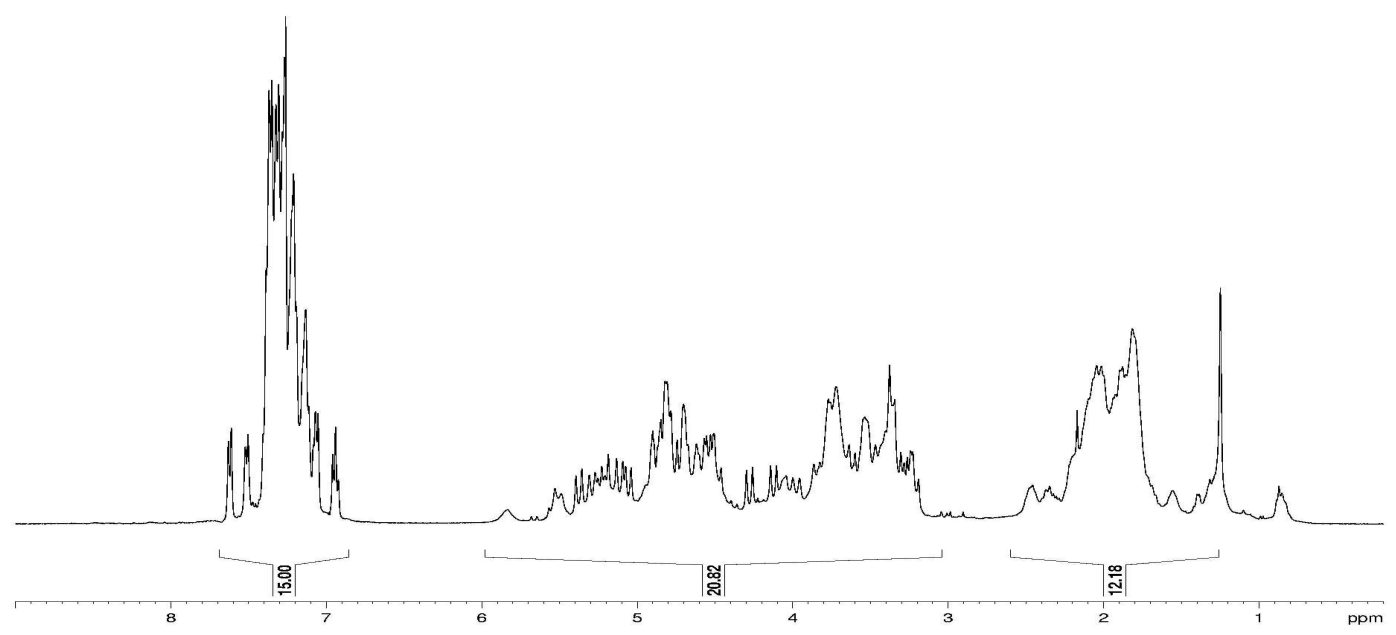

${ }^{13} \mathrm{C}$ NMR of compound $4 a\left(100 \mathrm{MHz}, \mathrm{CDCl}_{3}\right)$

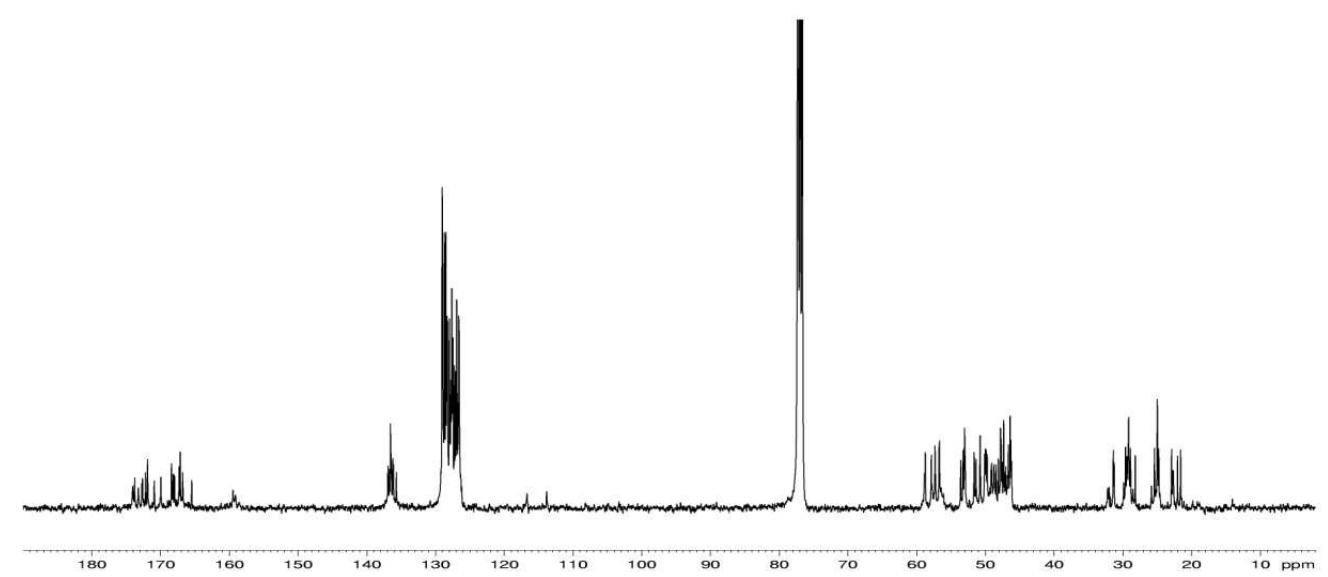




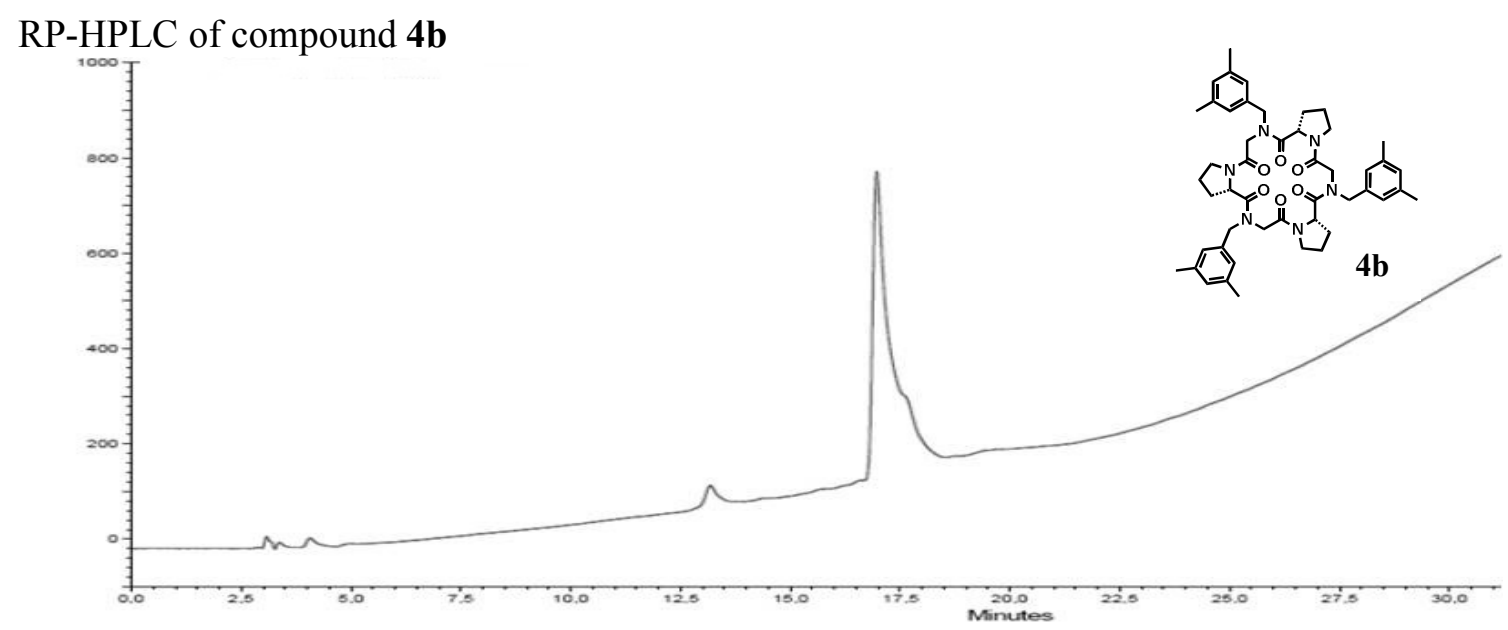

${ }^{1} \mathrm{H}$ NMR of compound $\mathbf{4 b}\left(400 \mathrm{MHz}, \mathrm{CDCl}_{3}\right)$

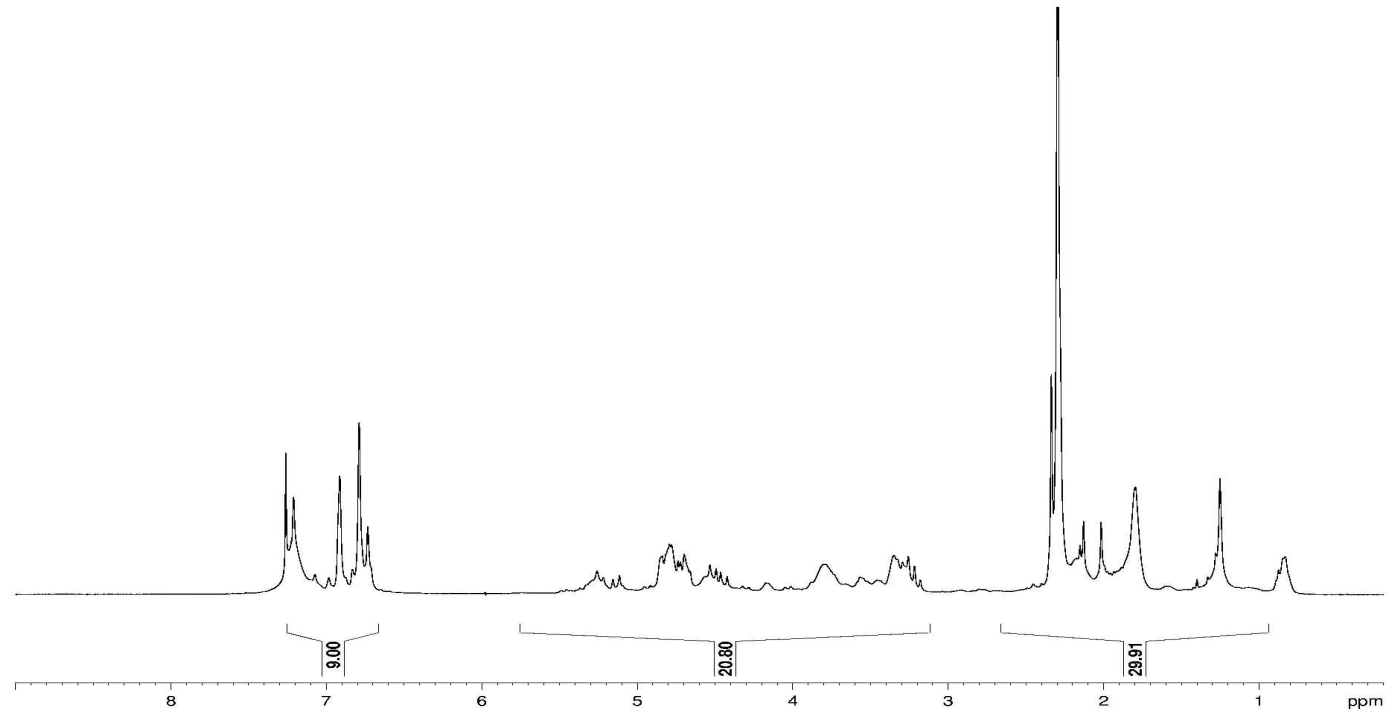

${ }^{13} \mathrm{C}$ NMR of compound $\mathbf{4 b}\left(100 \mathrm{MHz}, \mathrm{CDCl}_{3}\right)$

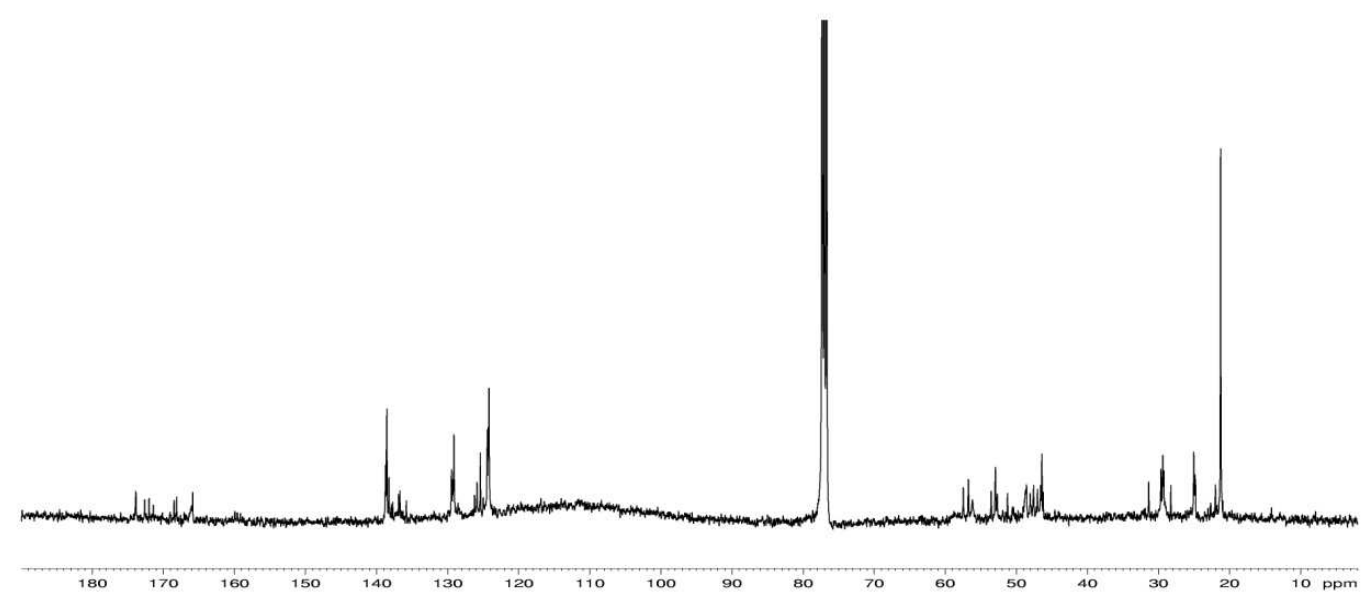




\section{RP-HPLC of compound 4c}

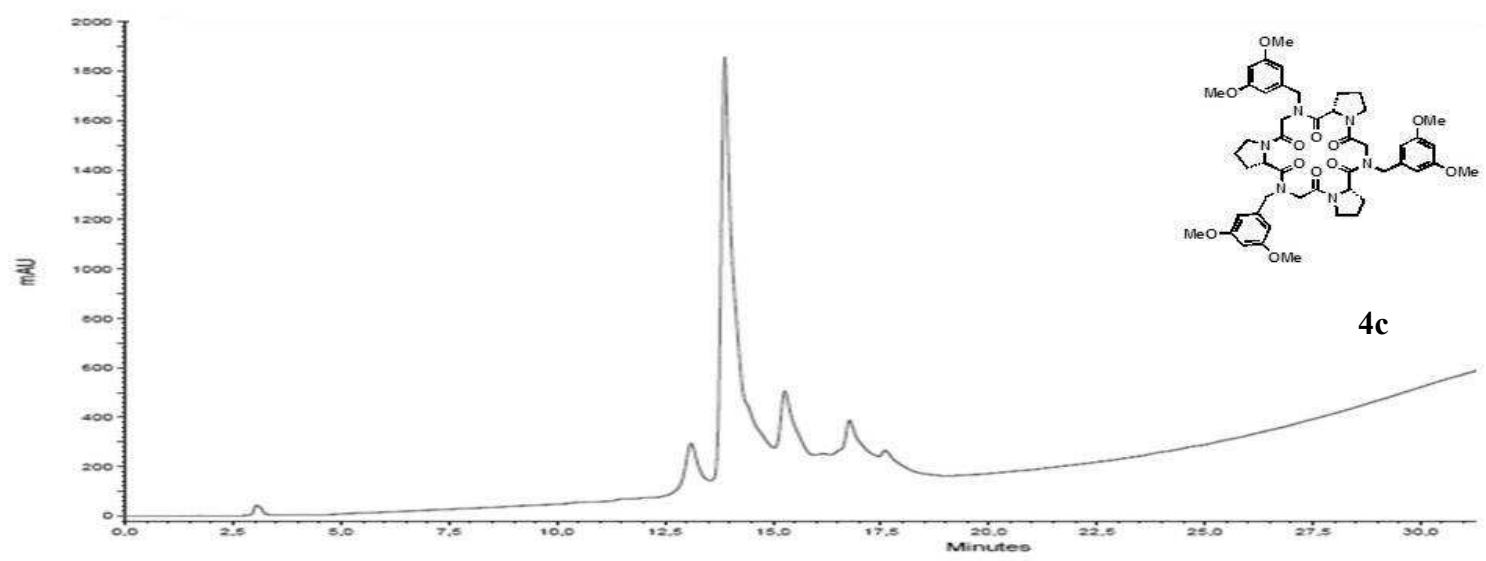

${ }^{1} \mathrm{H}$ NMR of compound $4 \mathbf{c}\left(300 \mathrm{MHz}, \mathrm{CDCl}_{3}\right)$

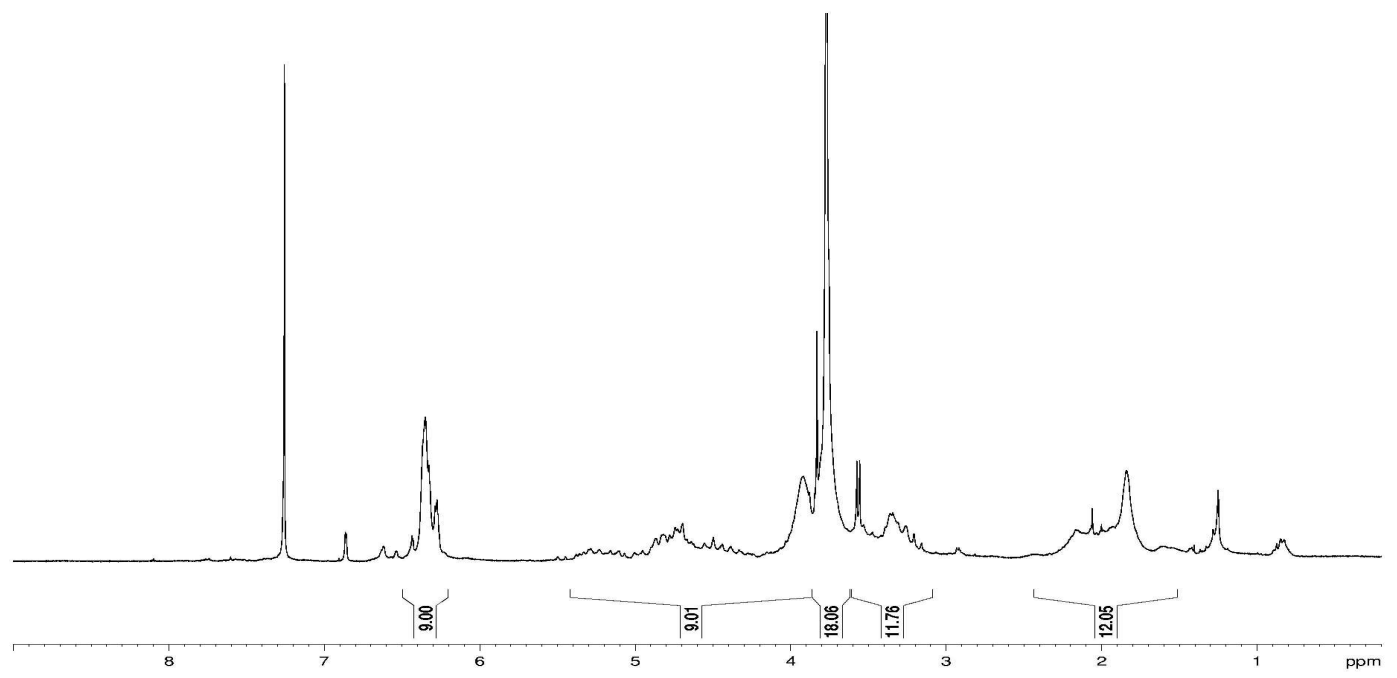

${ }^{13} \mathrm{C}$ NMR of compound $4 \mathbf{c}\left(100 \mathrm{MHz}, \mathrm{CDCl}_{3}\right)$

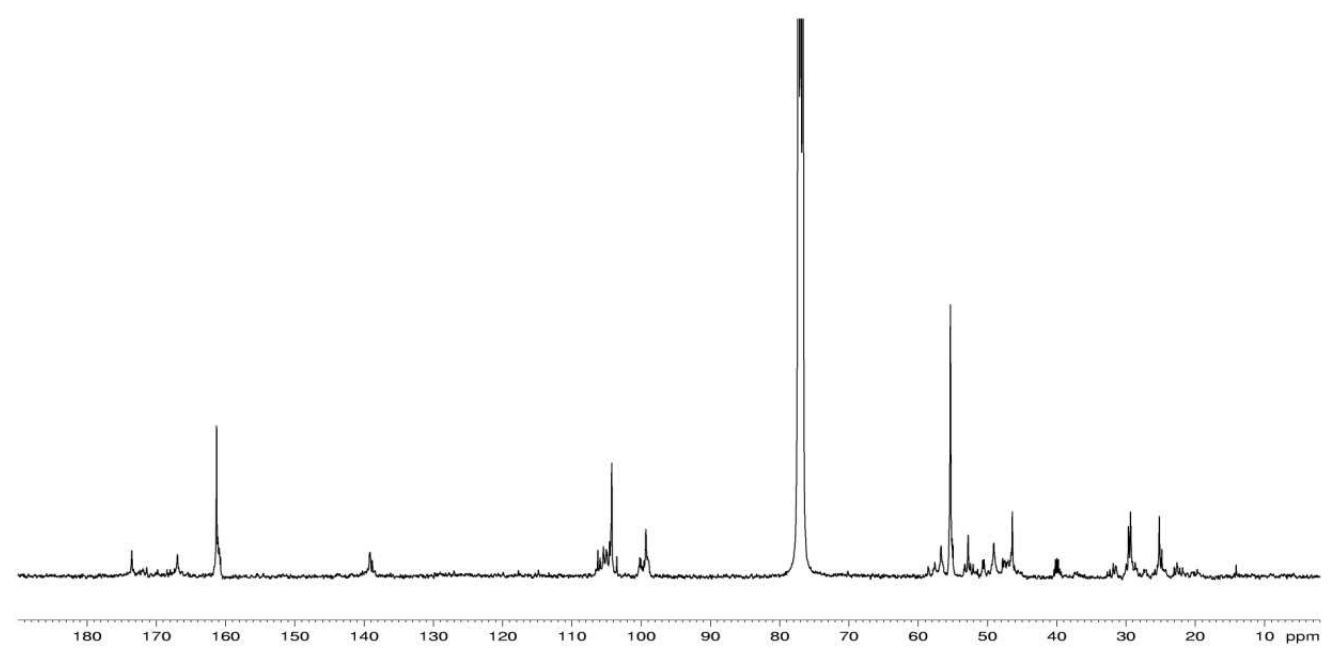


RP-HPLC of compound 4d

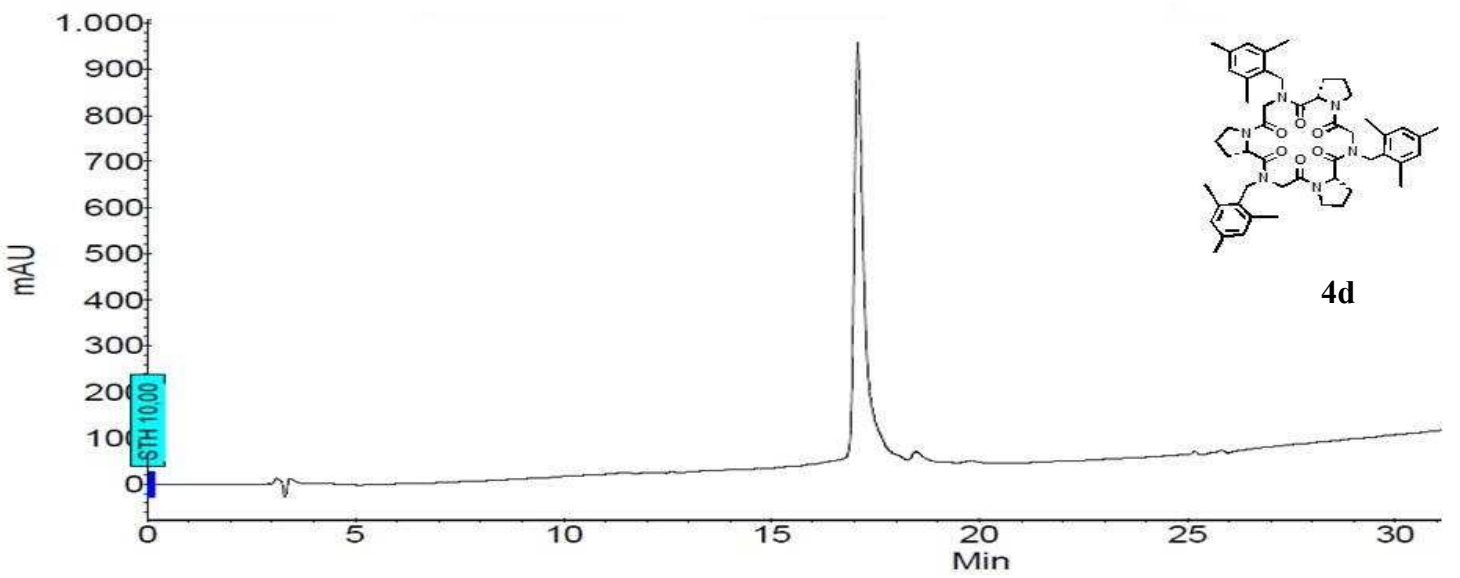

${ }^{1} \mathrm{H}$ NMR of compound $4 \mathbf{d}\left(400 \mathrm{MHz}, \mathrm{CDCl}_{3}\right)$

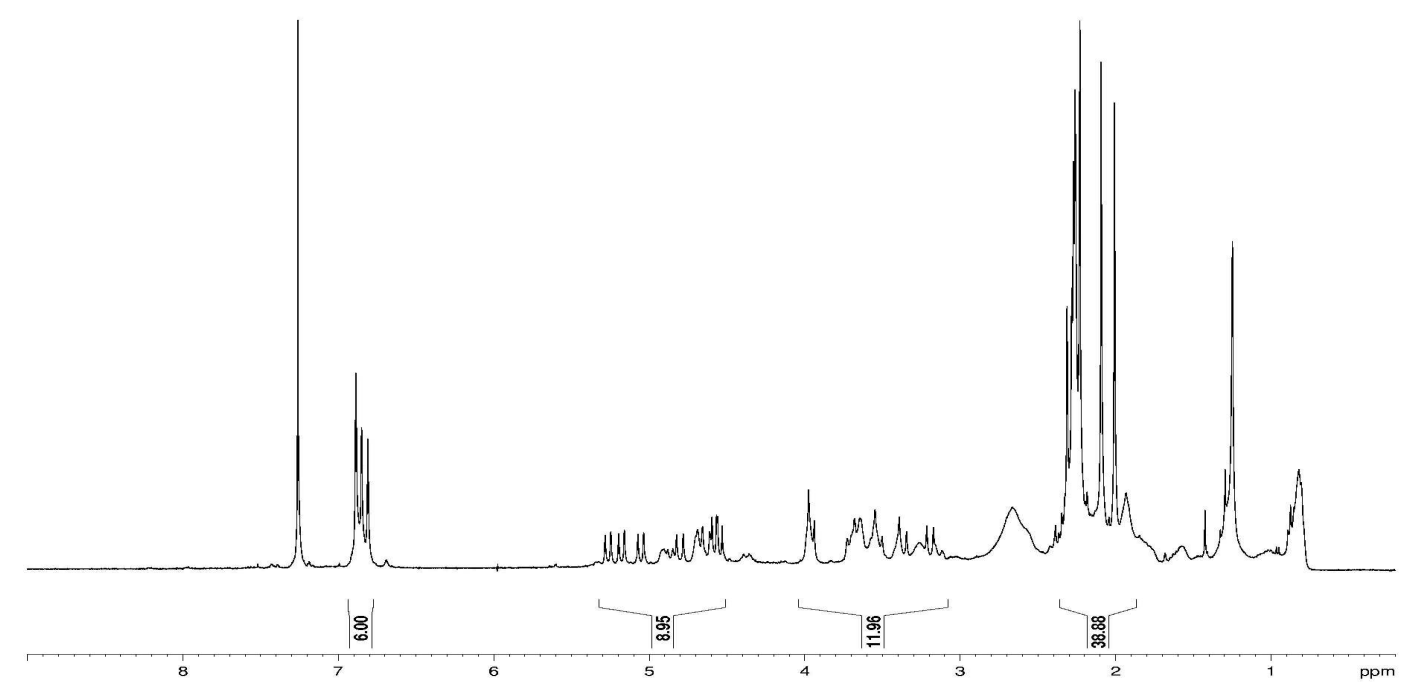

${ }^{13} \mathrm{C}$ NMR of compound $4 d\left(150 \mathrm{MHz}, \mathrm{CDCl}_{3}\right)$

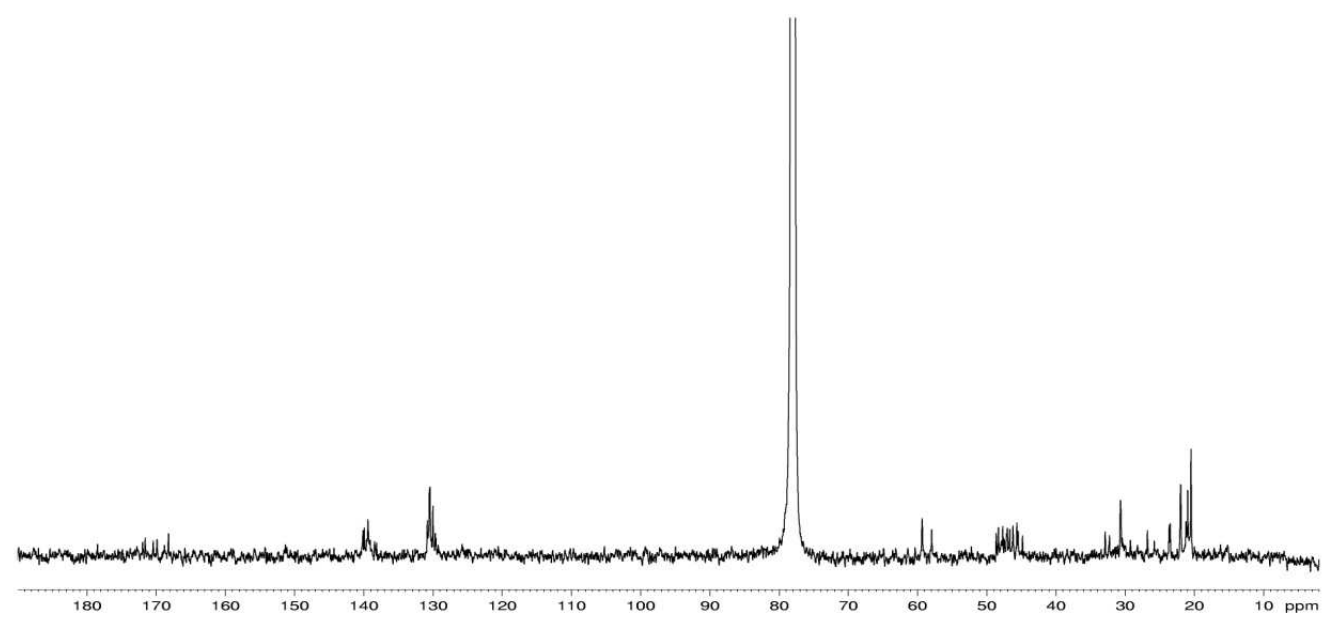




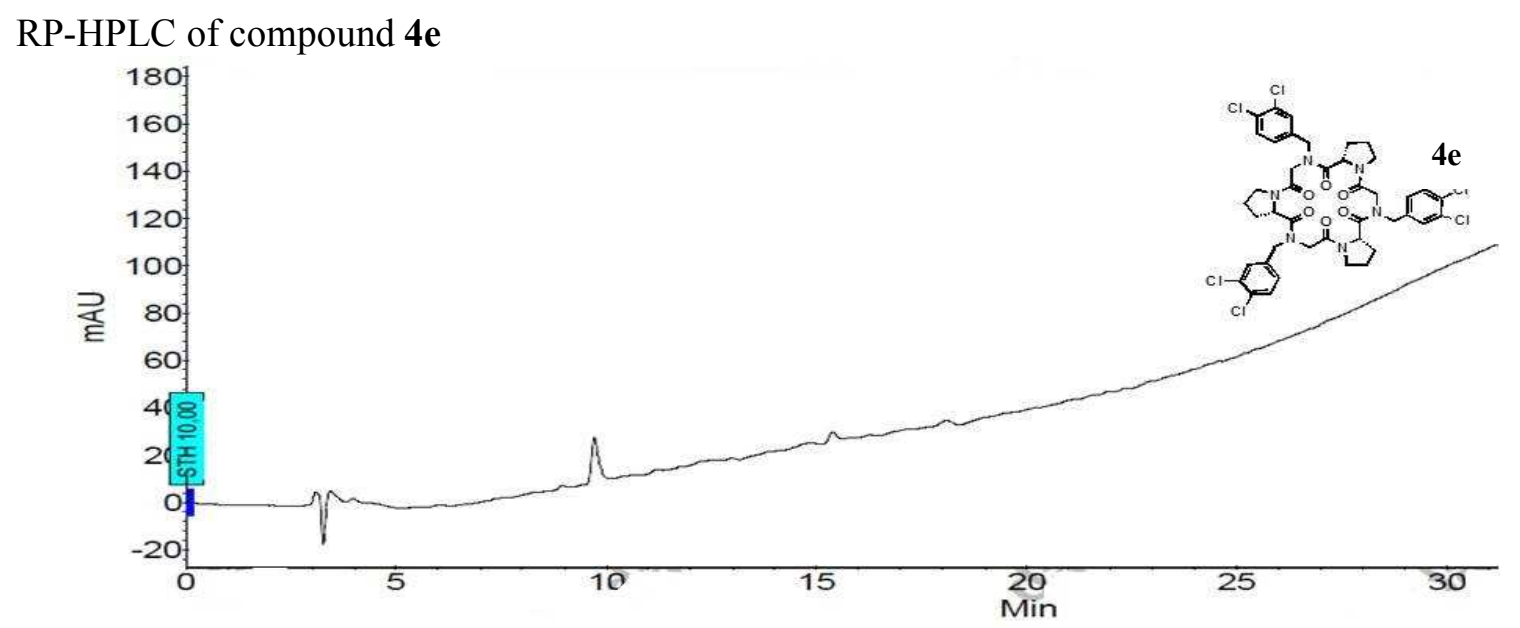

${ }^{1} \mathrm{H}$ NMR of compound $4 \mathbf{e}\left(600 \mathrm{MHz}, \mathrm{CDCl}_{3}\right)$

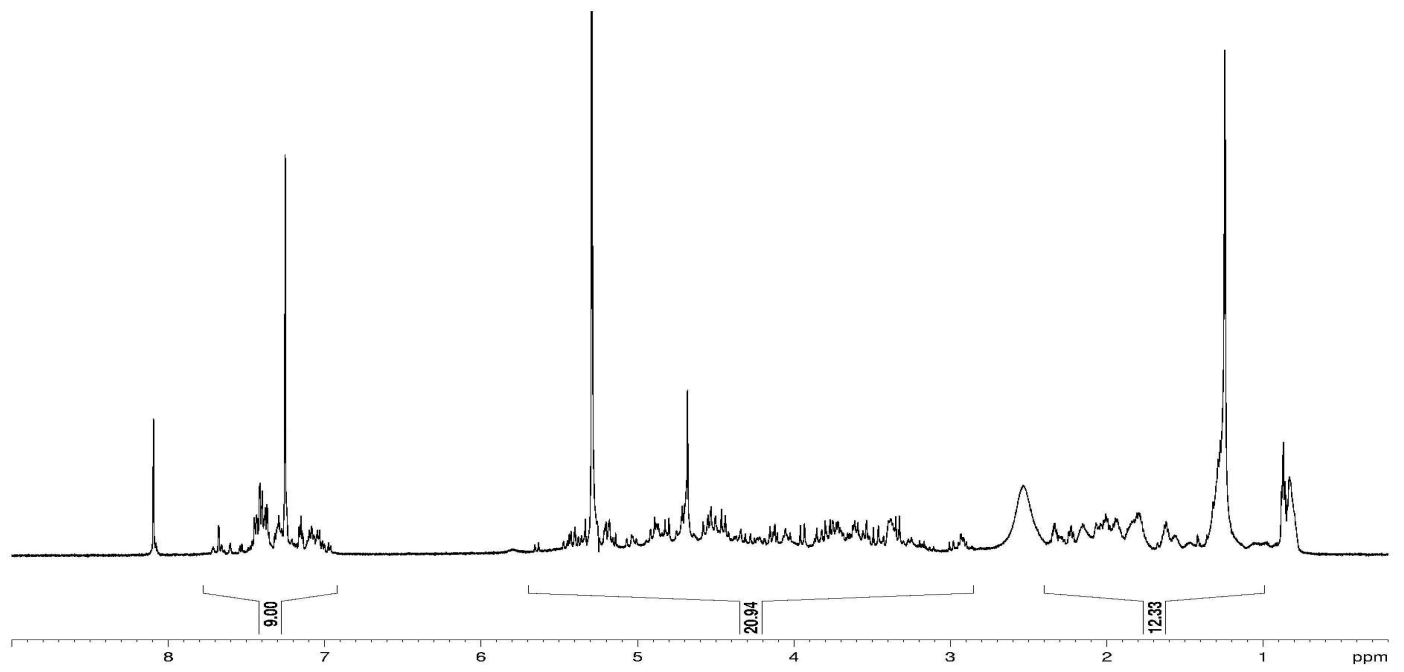

${ }^{13} \mathrm{C}$ NMR of compound $4 \mathbf{e}\left(150 \mathrm{MHz}, \mathrm{CDCl}_{3}\right)$

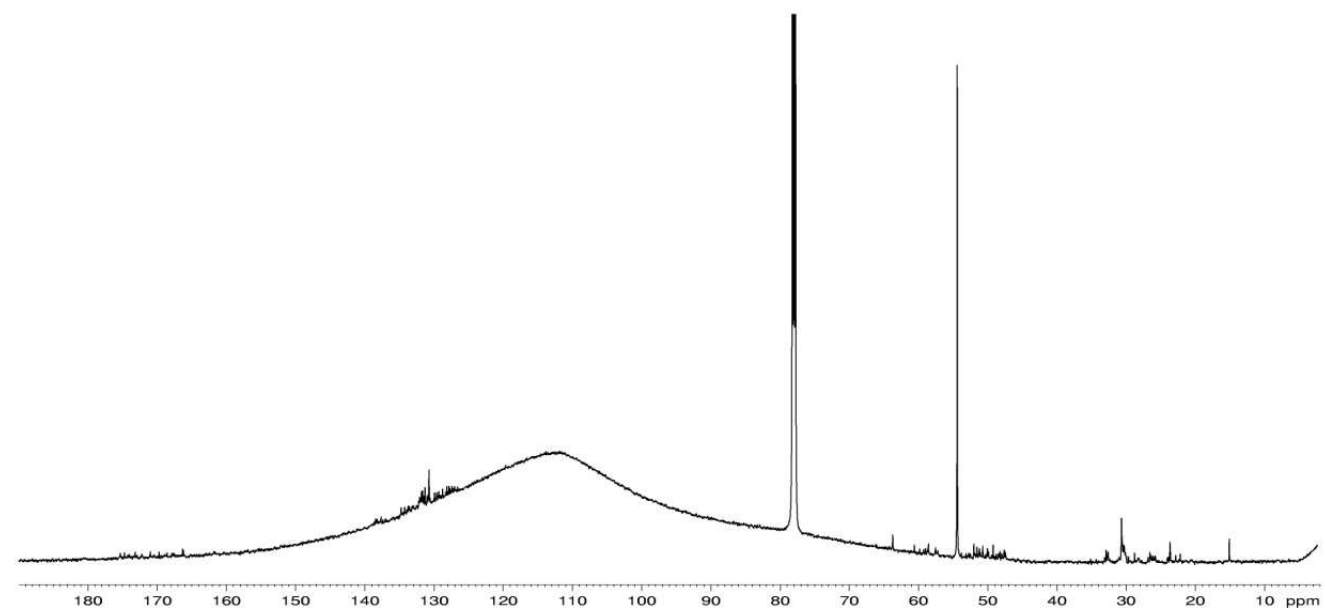




\section{RP-HPLC of compound $\mathbf{4 f}$}

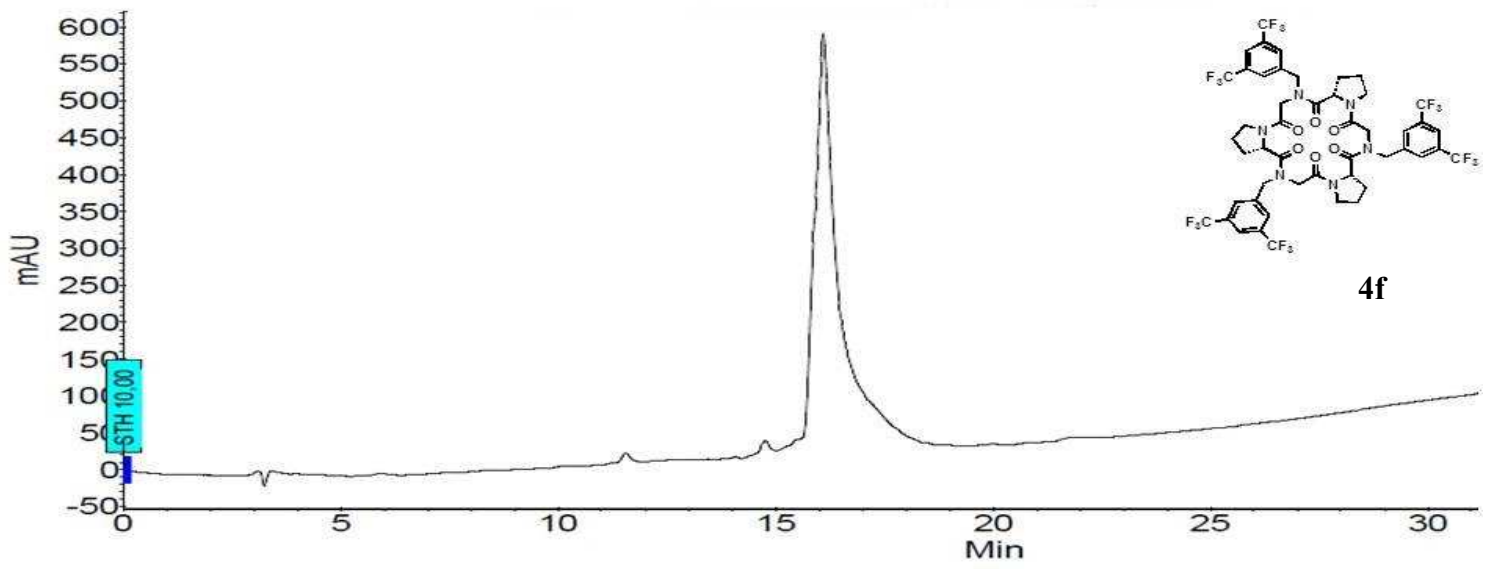

${ }^{1} \mathrm{H}$ NMR of compound $4 \mathbf{f}\left(400 \mathrm{MHz}, \mathrm{CDCl}_{3}\right)$

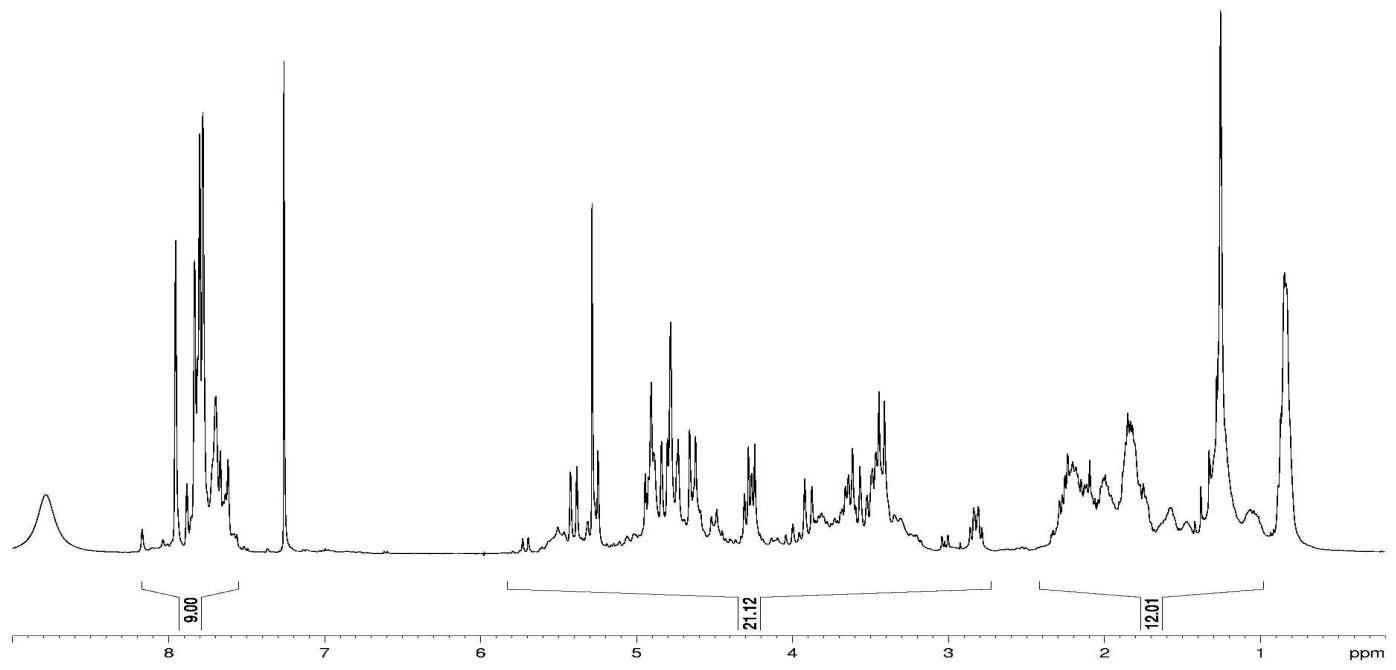

${ }^{13} \mathrm{C}$ NMR of compound $\mathbf{4 f}\left(100 \mathrm{MHz}, \mathrm{CDCl}_{3}\right)$

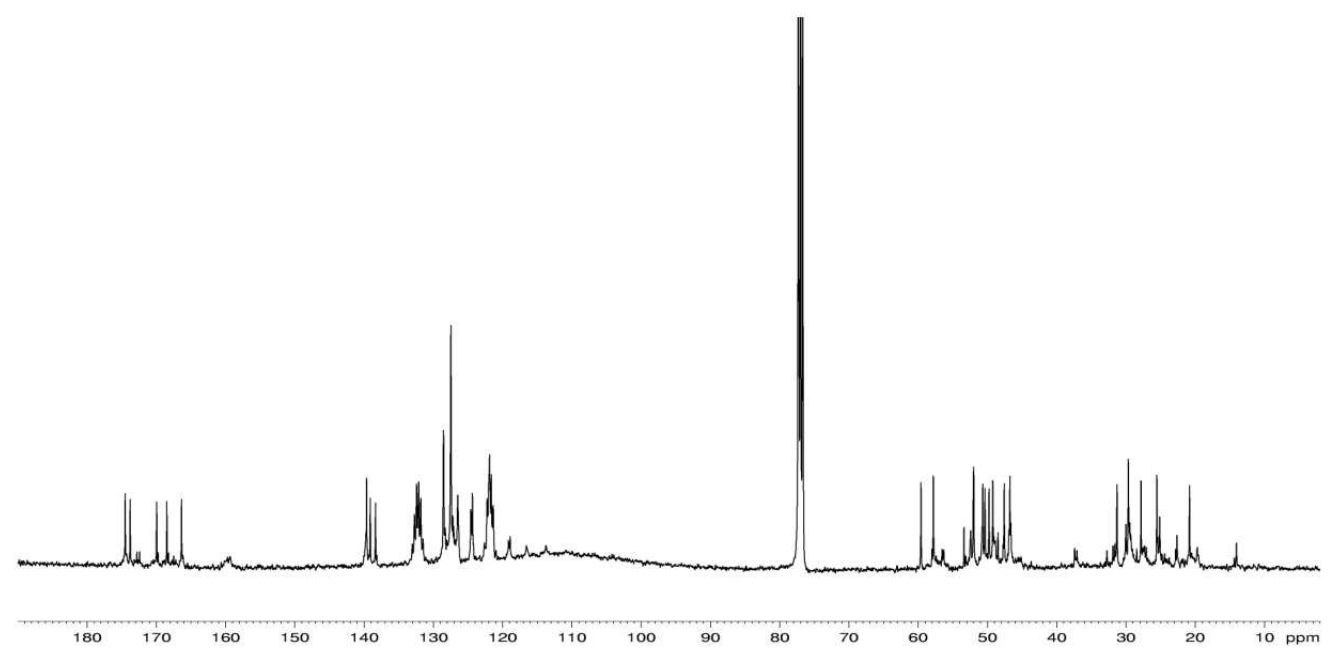




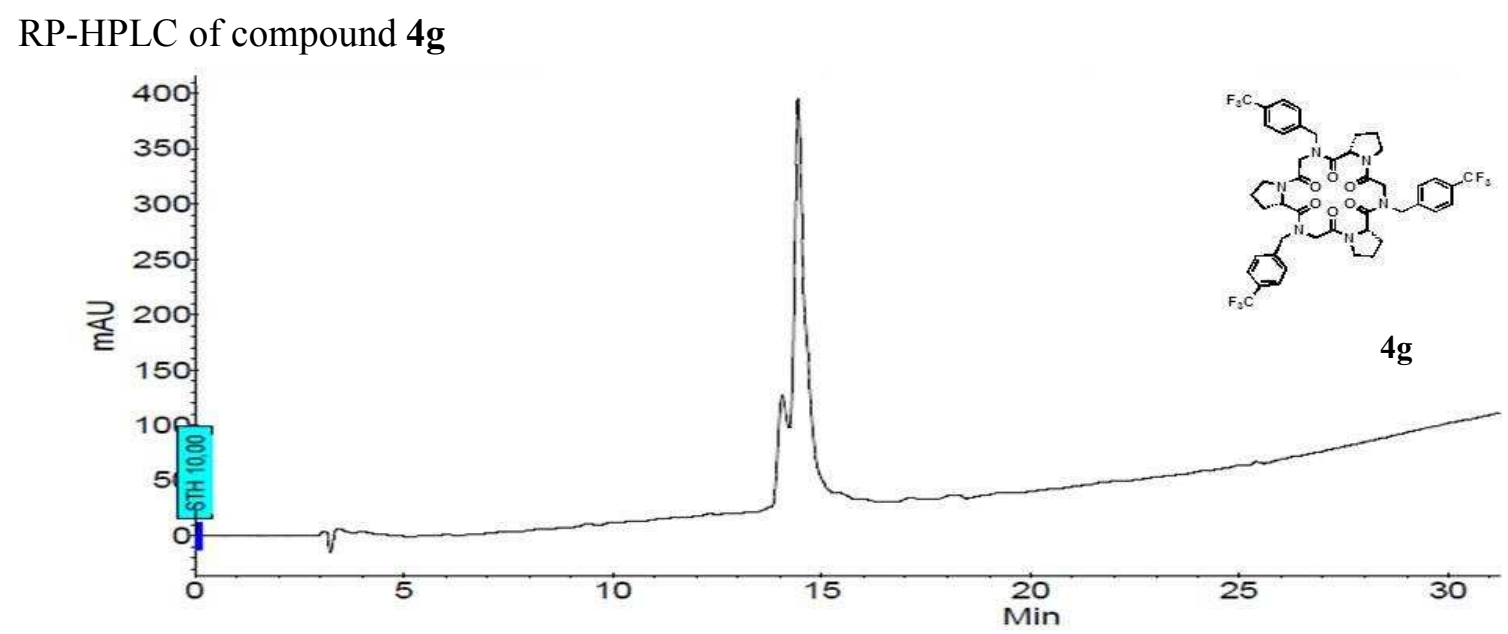

${ }^{1} \mathrm{H}$ NMR of compound $\mathbf{4 g}\left(400 \mathrm{MHz}, \mathrm{CDCl}_{3}\right)$

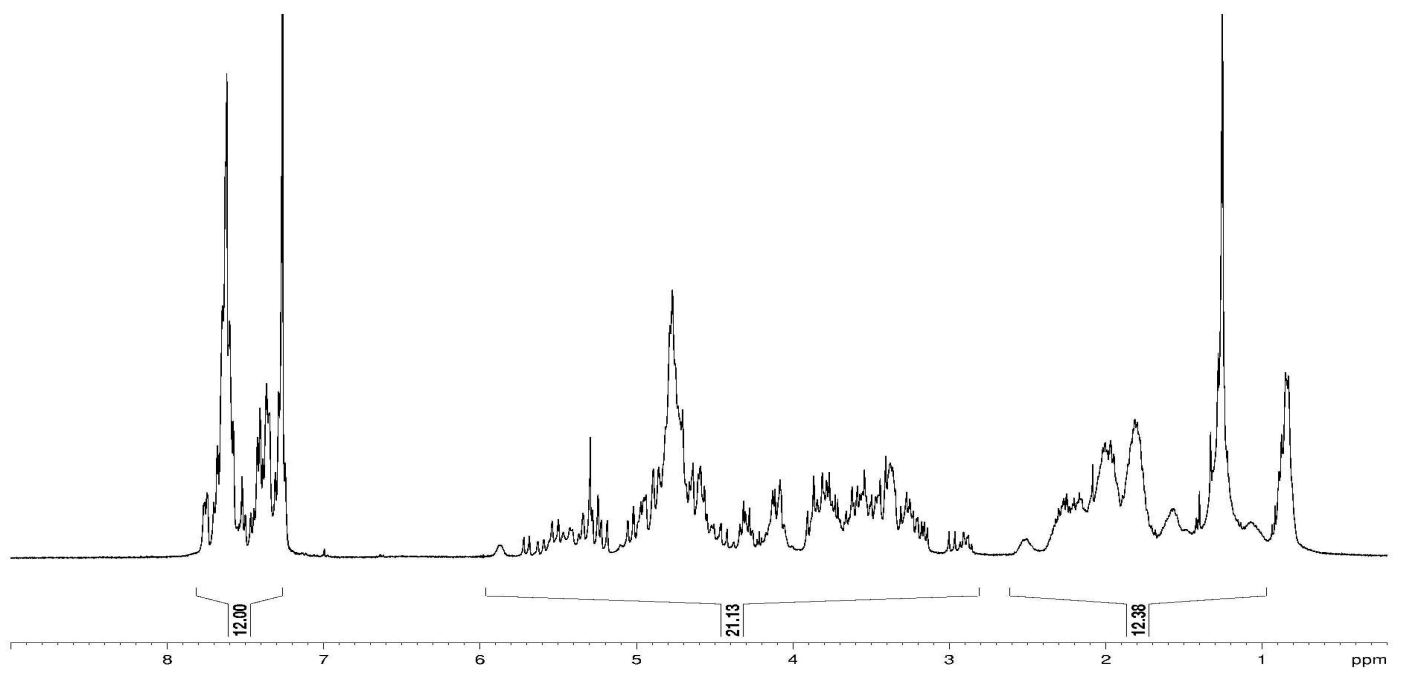

${ }^{13} \mathrm{C}$ NMR of compound $\mathbf{4 g}\left(100 \mathrm{MHz}, \mathrm{CDCl}_{3}\right)$

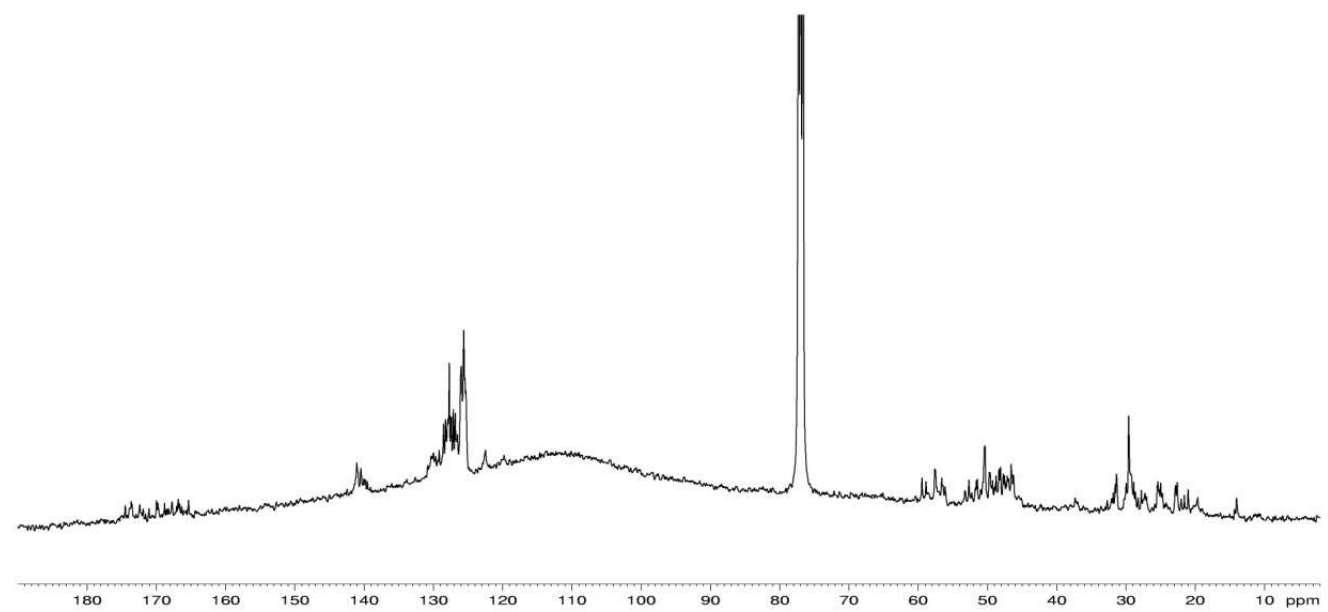




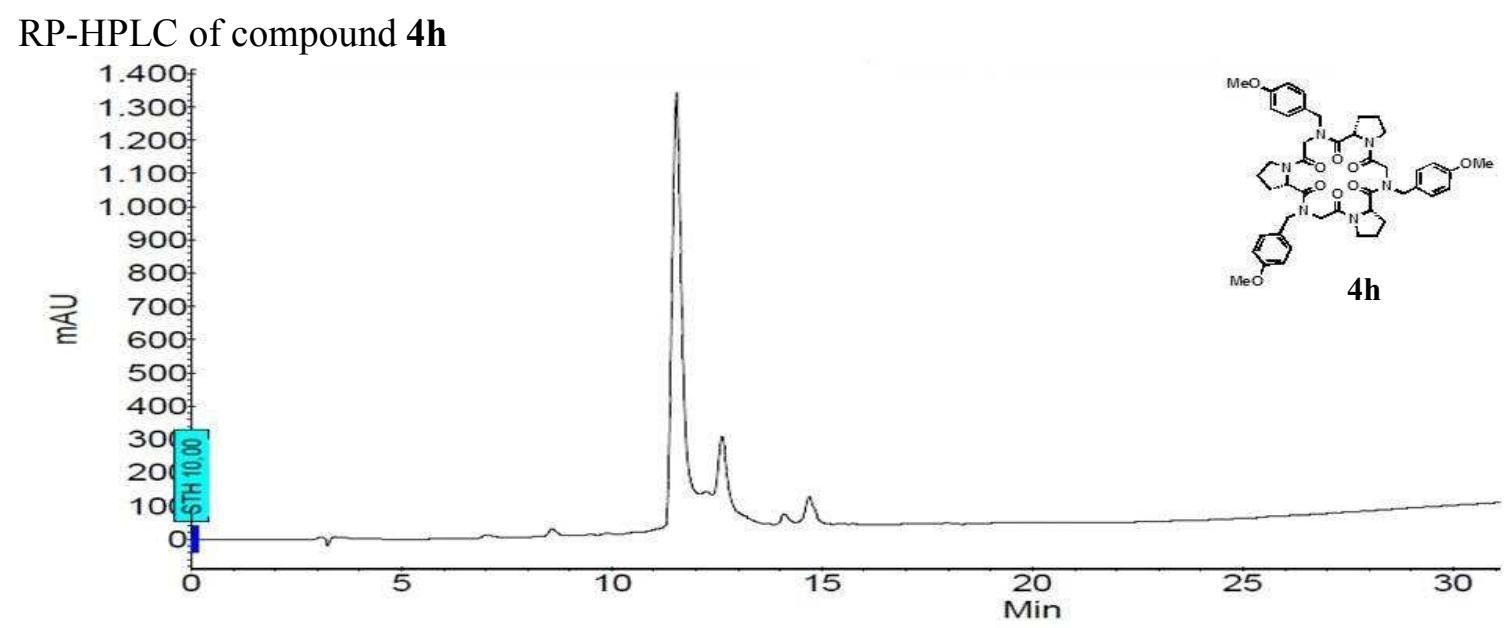

${ }^{1} \mathrm{H}$ NMR of compound $\mathbf{4 h}\left(300 \mathrm{MHz}, \mathrm{CDCl}_{3}\right)$

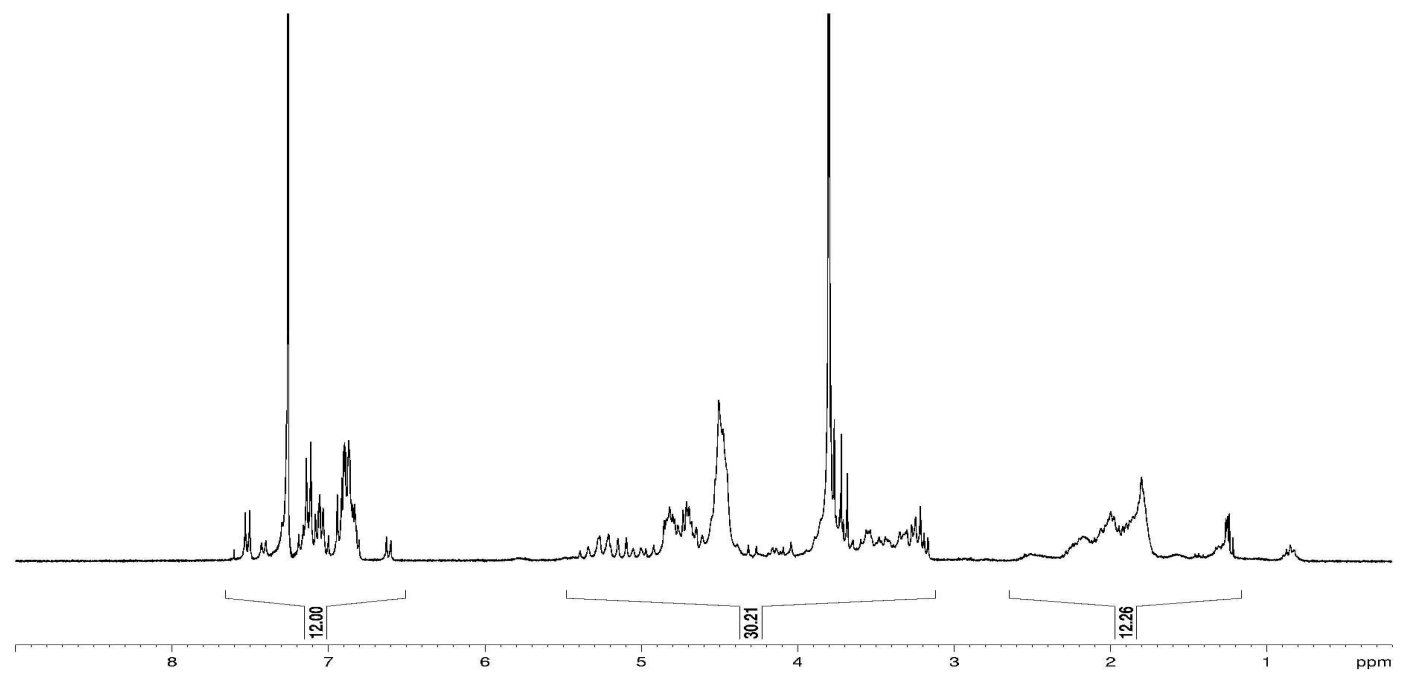

${ }^{13} \mathrm{C}$ NMR of compound $\mathbf{4 h}\left(100 \mathrm{MHz}, \mathrm{CDCl}_{3}\right)$

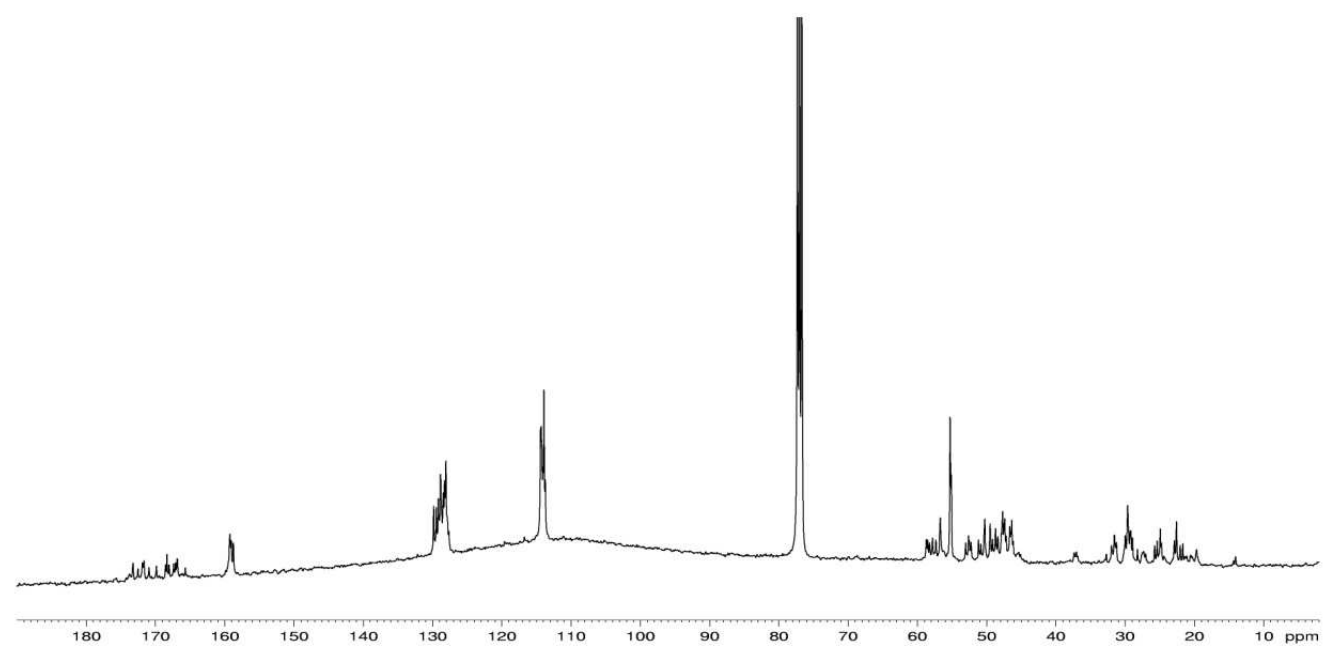


RP-HPLC of compound 4i

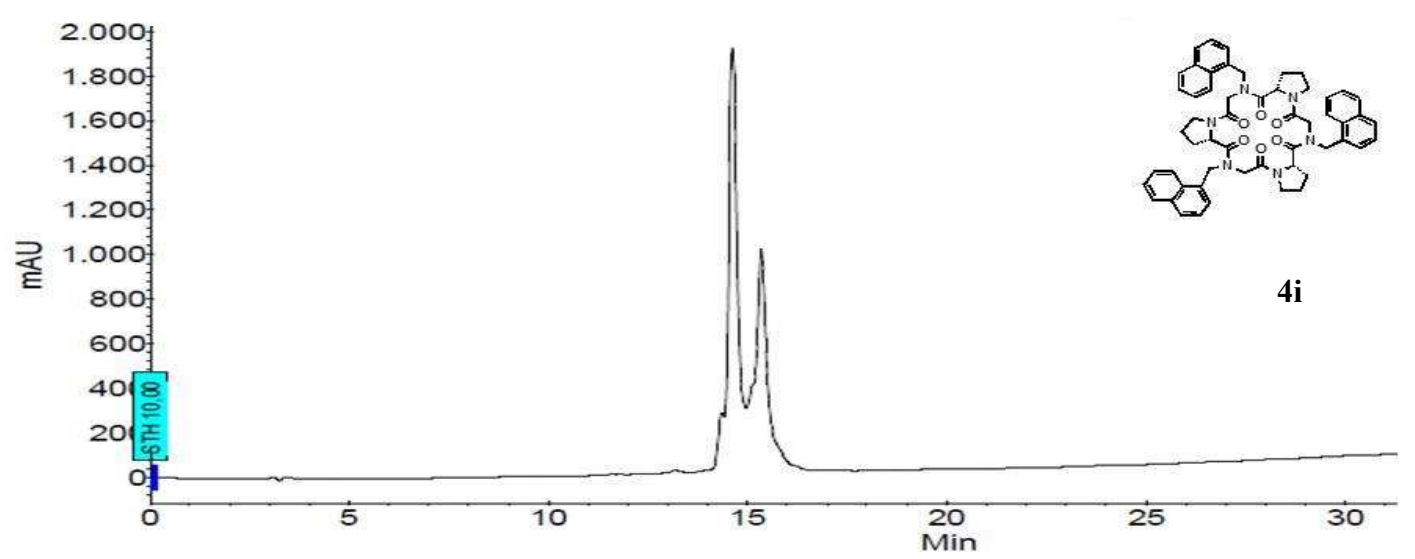

${ }^{1} \mathrm{H}$ NMR of compound $4 \mathbf{i}\left(400 \mathrm{MHz}, \mathrm{CDCl}_{3}\right.$ )

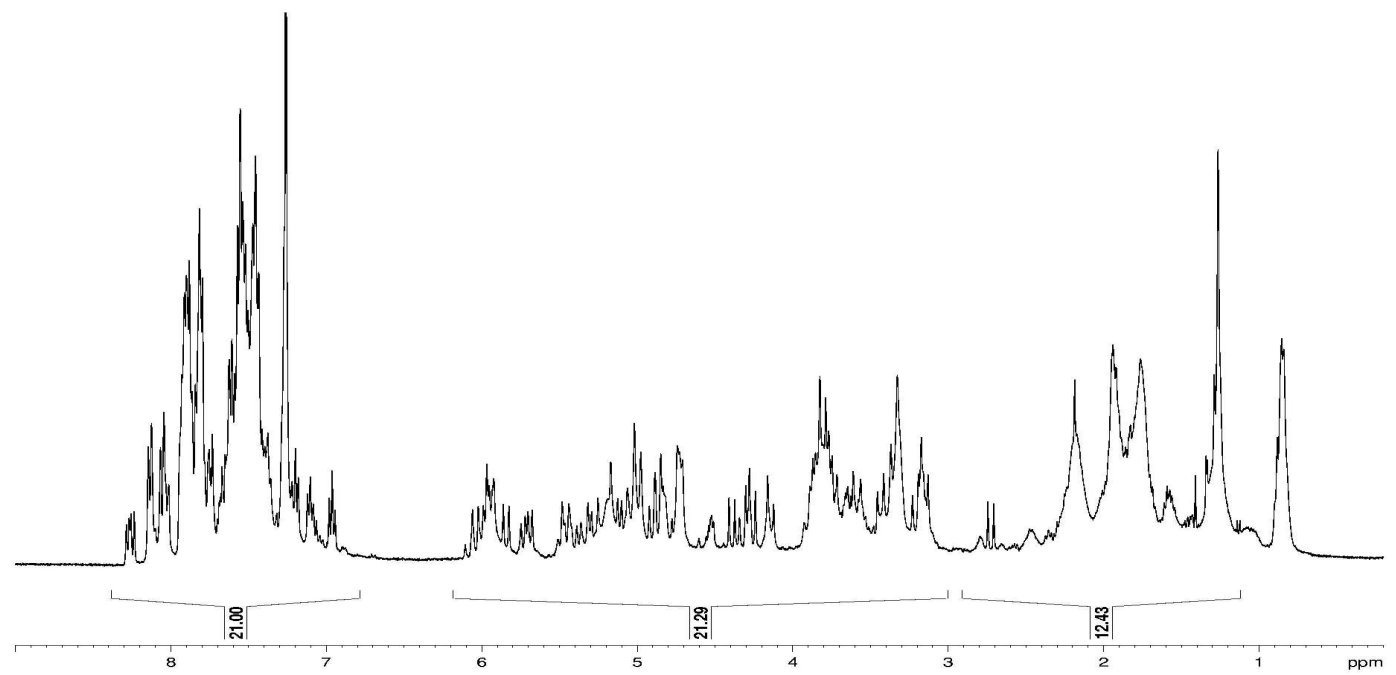

${ }^{13} \mathrm{C}$ NMR of compound $4 \mathbf{i}\left(100 \mathrm{MHz}, \mathrm{CDCl}_{3}\right)$

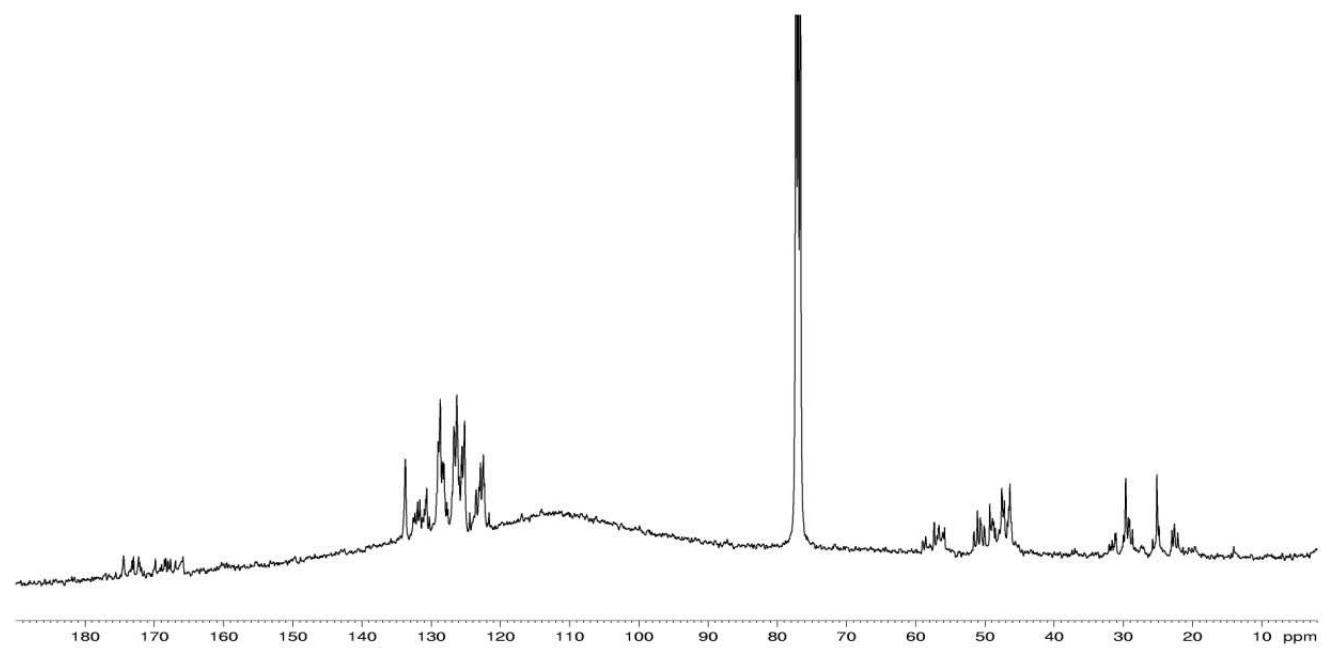


RP-HPLC of compound 7

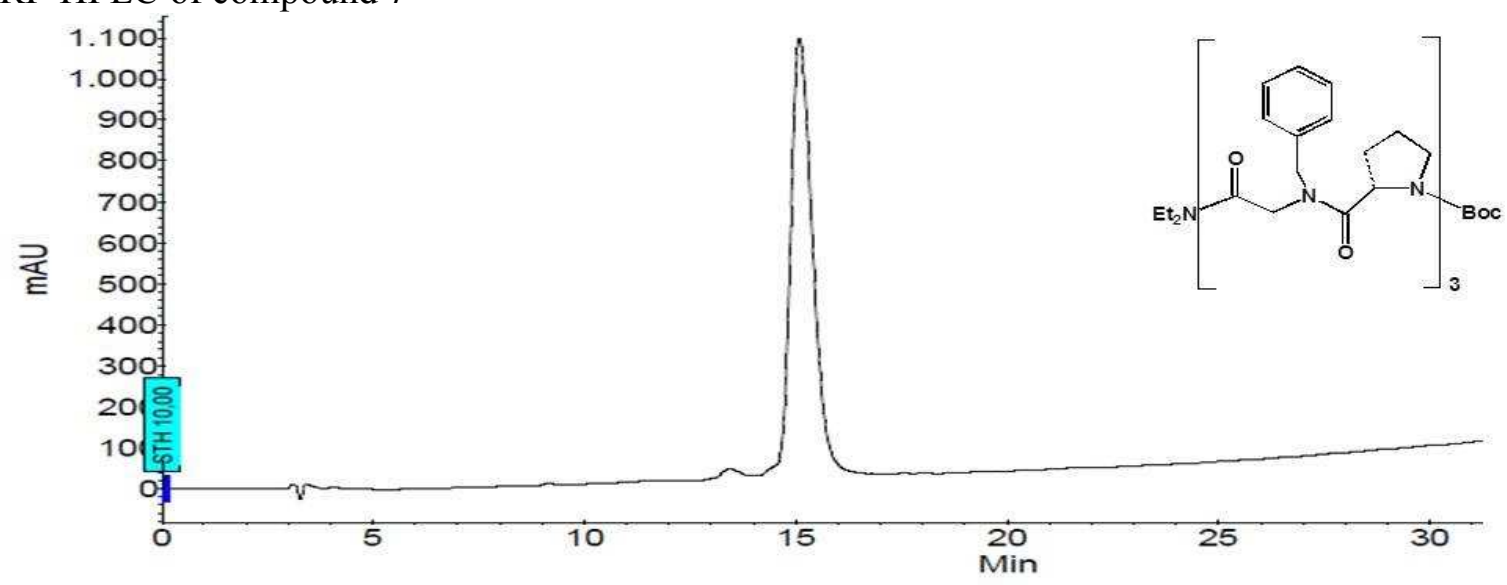

${ }^{1} \mathrm{H}$ NMR of compound 7 (400 MHz, $\mathrm{CDCl}_{3}$ )

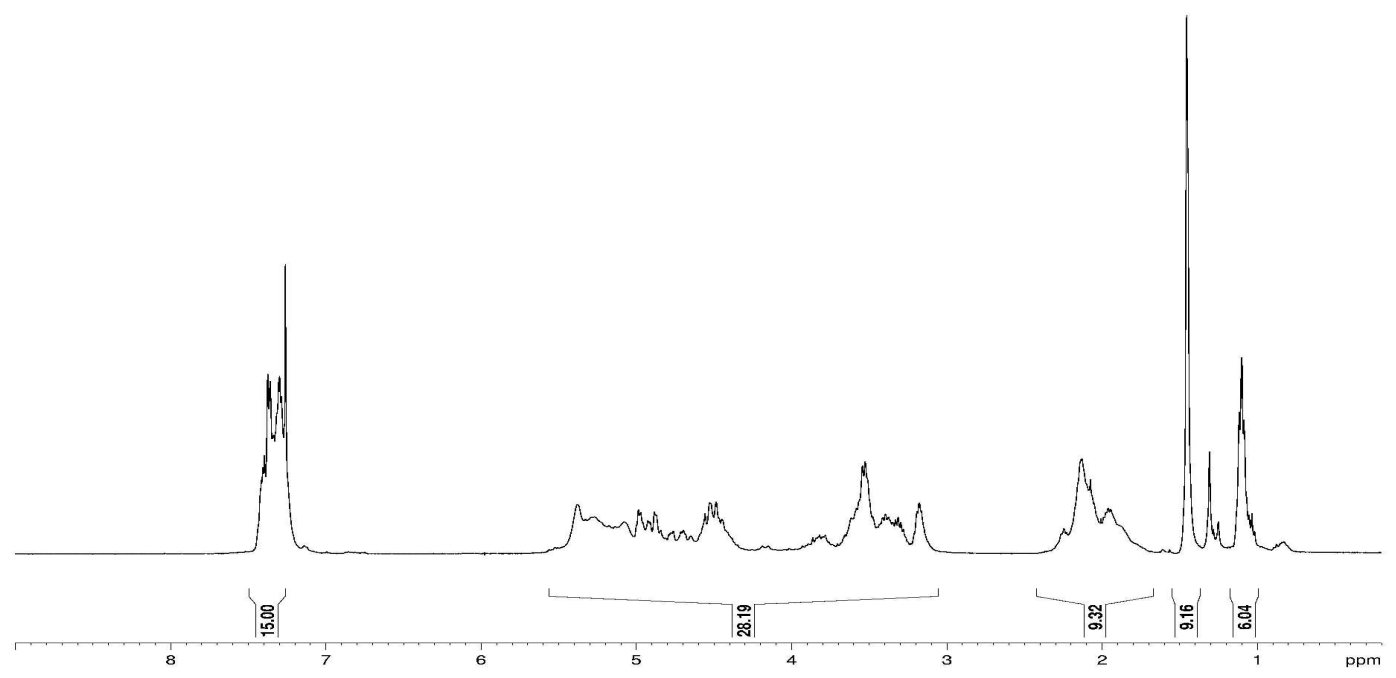

${ }^{13} \mathrm{C}$ NMR of compound $7\left(150 \mathrm{MHz}, \mathrm{CDCl}_{3}\right)$

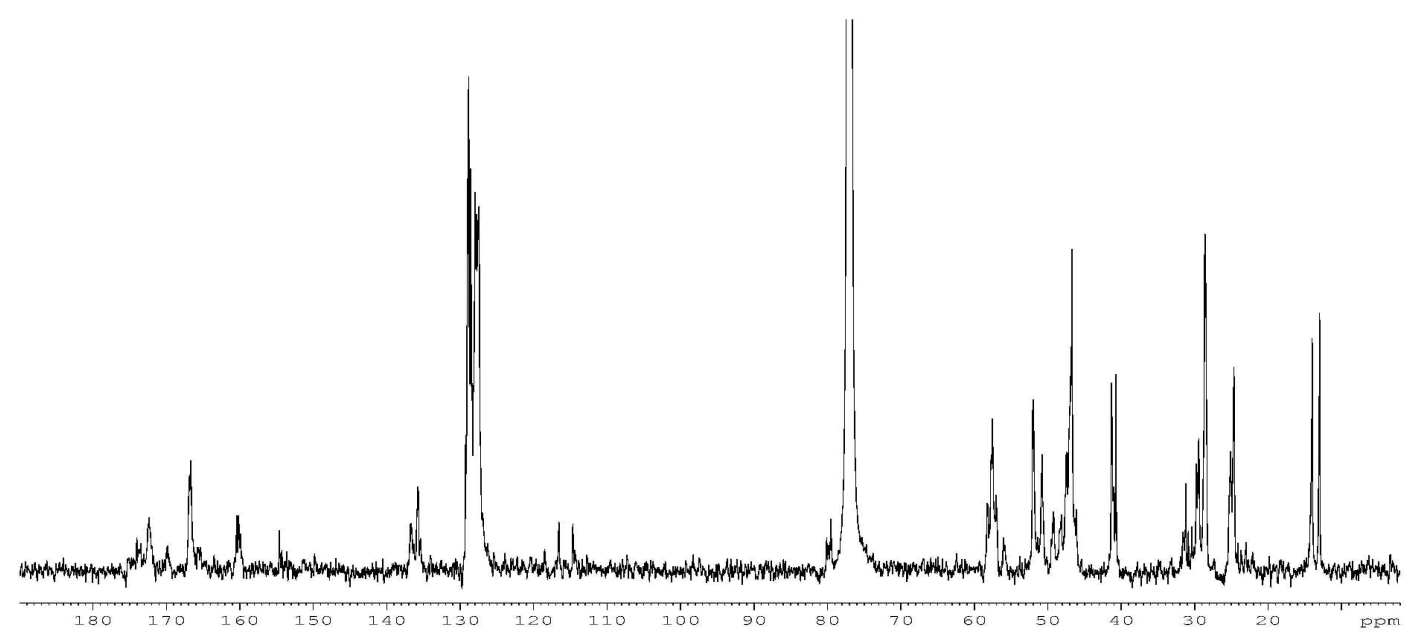




\section{2D NMR spectra of peptoid 3b}

${ }^{1} \mathrm{H}-{ }^{1} \mathrm{H}$ COSY NMR of compound $\mathbf{3 b}\left(600 \mathrm{MHz}\right.$, DMSO- $\left.d_{6}\right)$

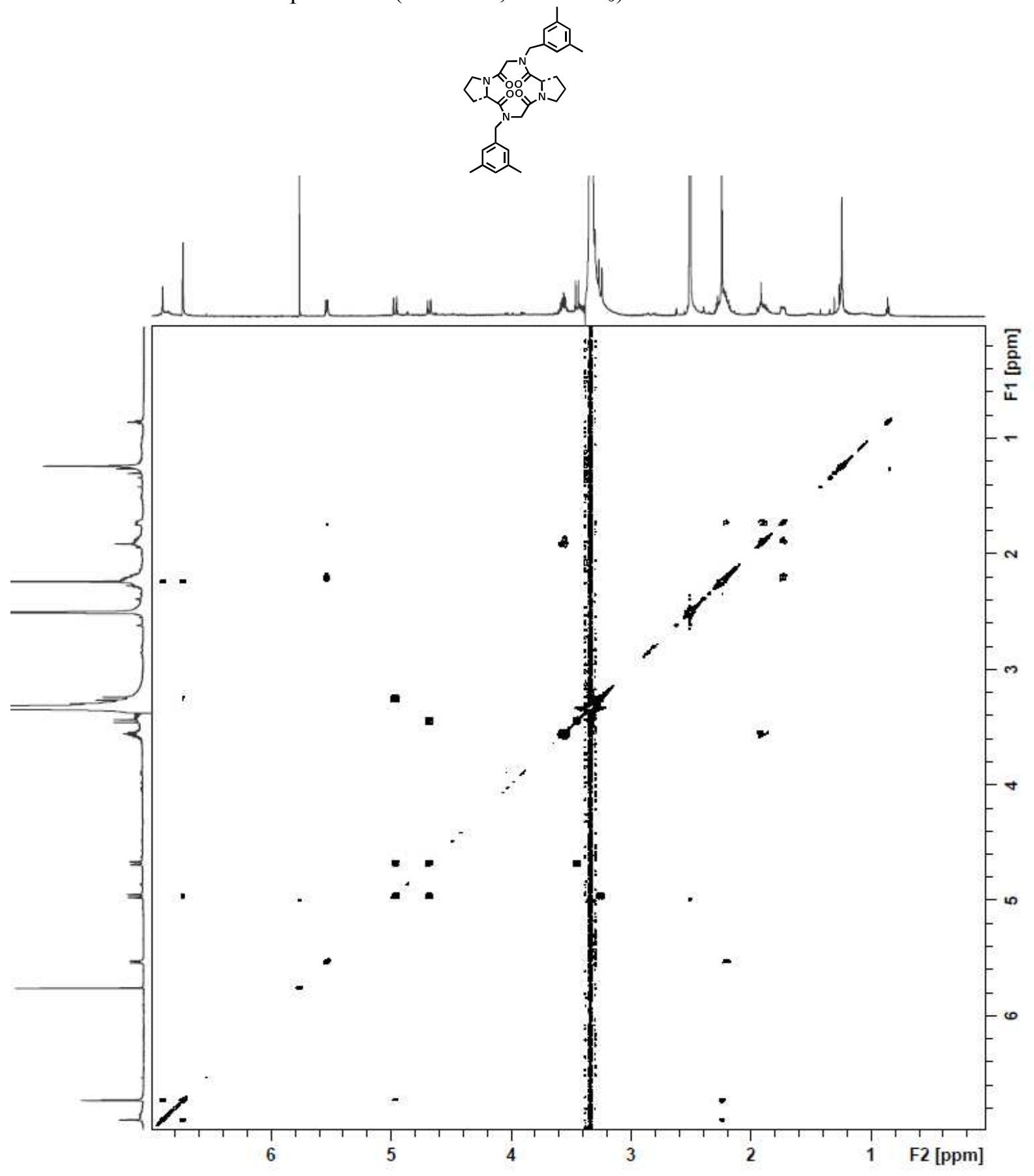


${ }^{1} \mathrm{H}-{ }^{1} \mathrm{H}$ ROESY NMR of compound $\mathbf{3 b}\left(600 \mathrm{MHz}\right.$, DMSO- $\left.d_{6}\right)$
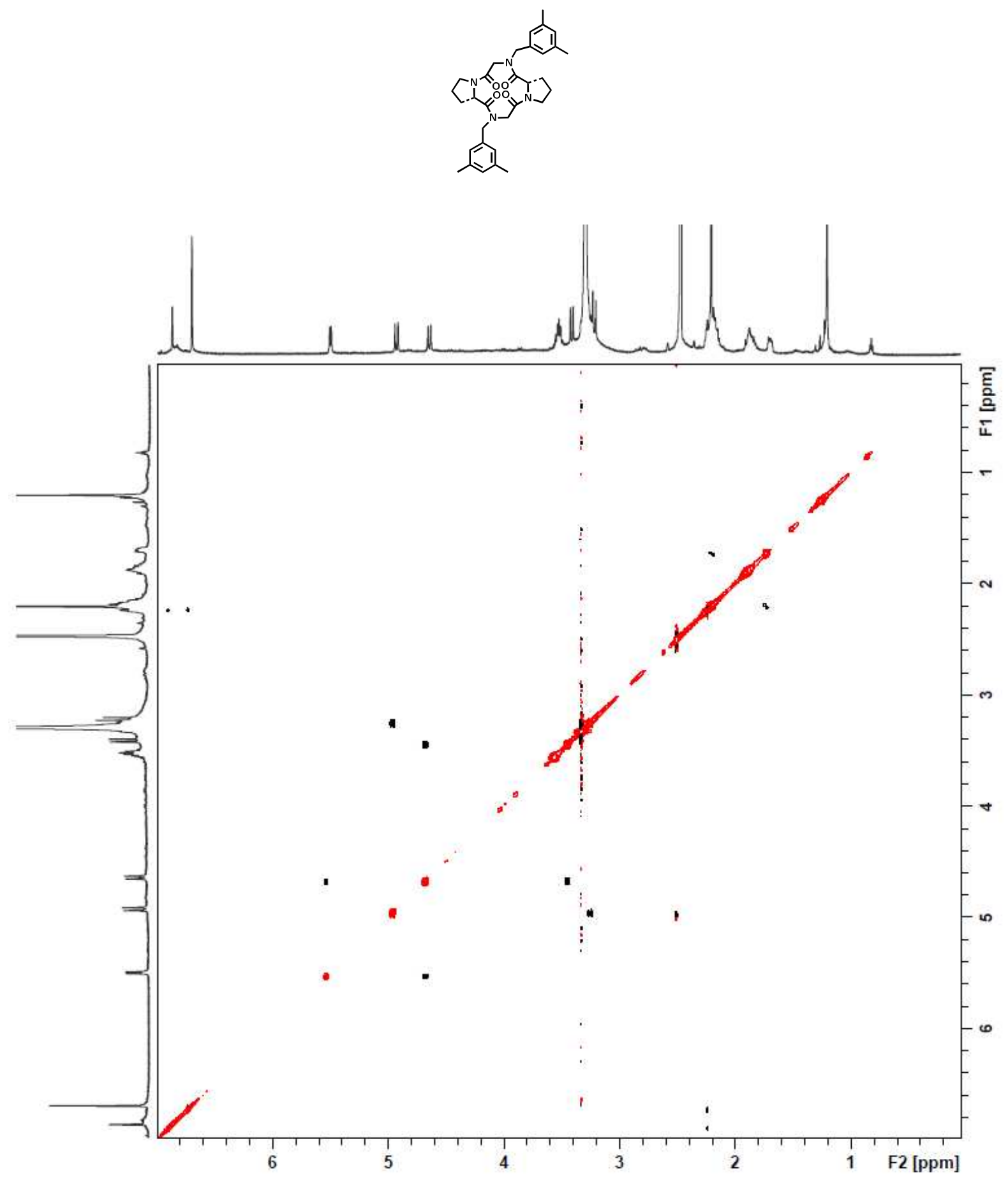
${ }^{1} \mathrm{H}-{ }^{13} \mathrm{C}$ HMQC NMR of compound $3 \mathbf{b}\left(600 \mathrm{MHz}\right.$, DMSO- $\left.d_{6}\right)$
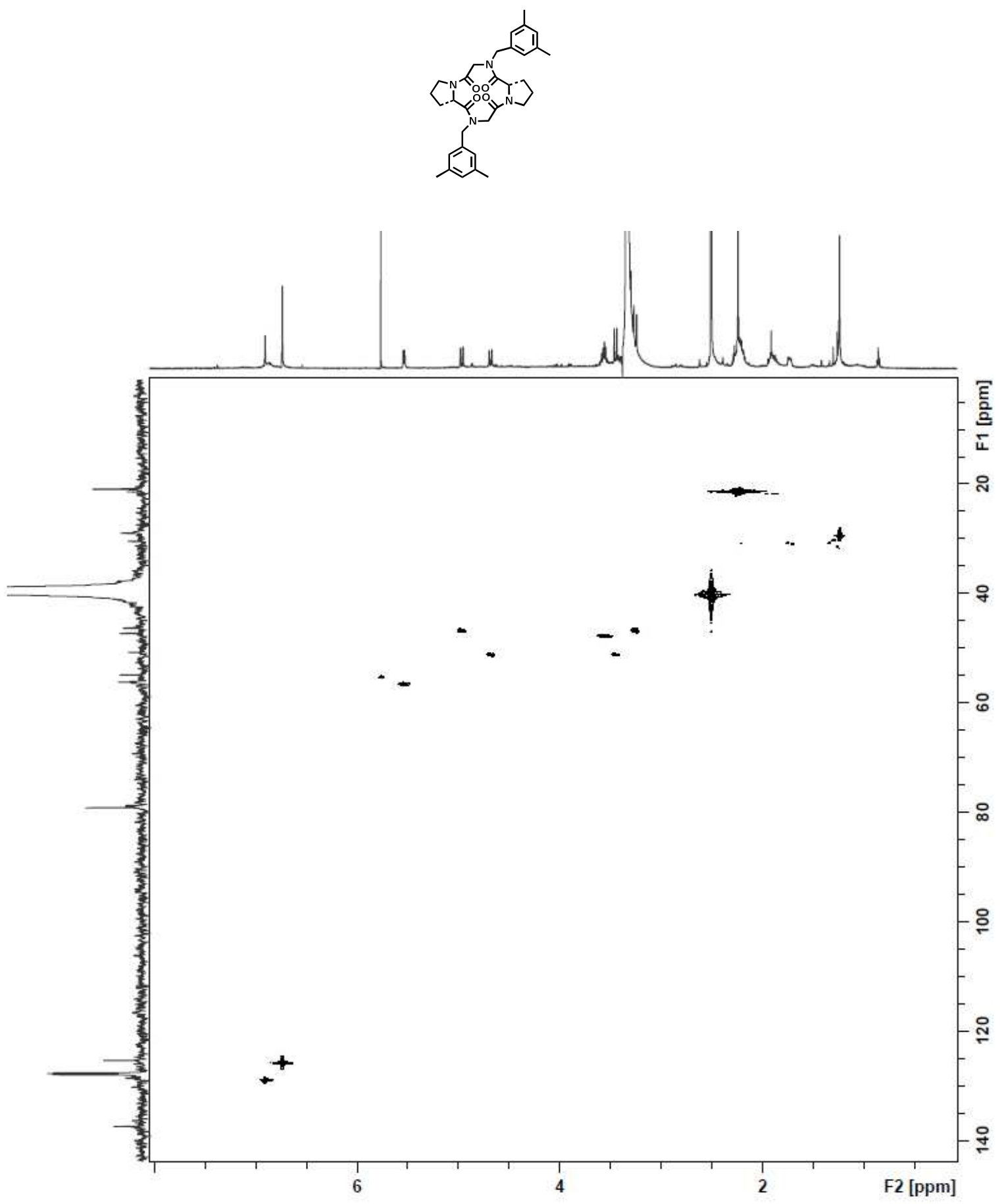


\section{7. ${ }^{1} \mathrm{H}$ NMR and ${ }^{13} \mathrm{C}$ NMR spectra of compounds $2 \mathrm{da}-2 \mathrm{do}$}

${ }^{1} \mathrm{H} \mathrm{NMR}$ of compound $2 \mathbf{d a}\left(400 \mathrm{MHz}, \mathrm{CDCl}_{3}\right)$

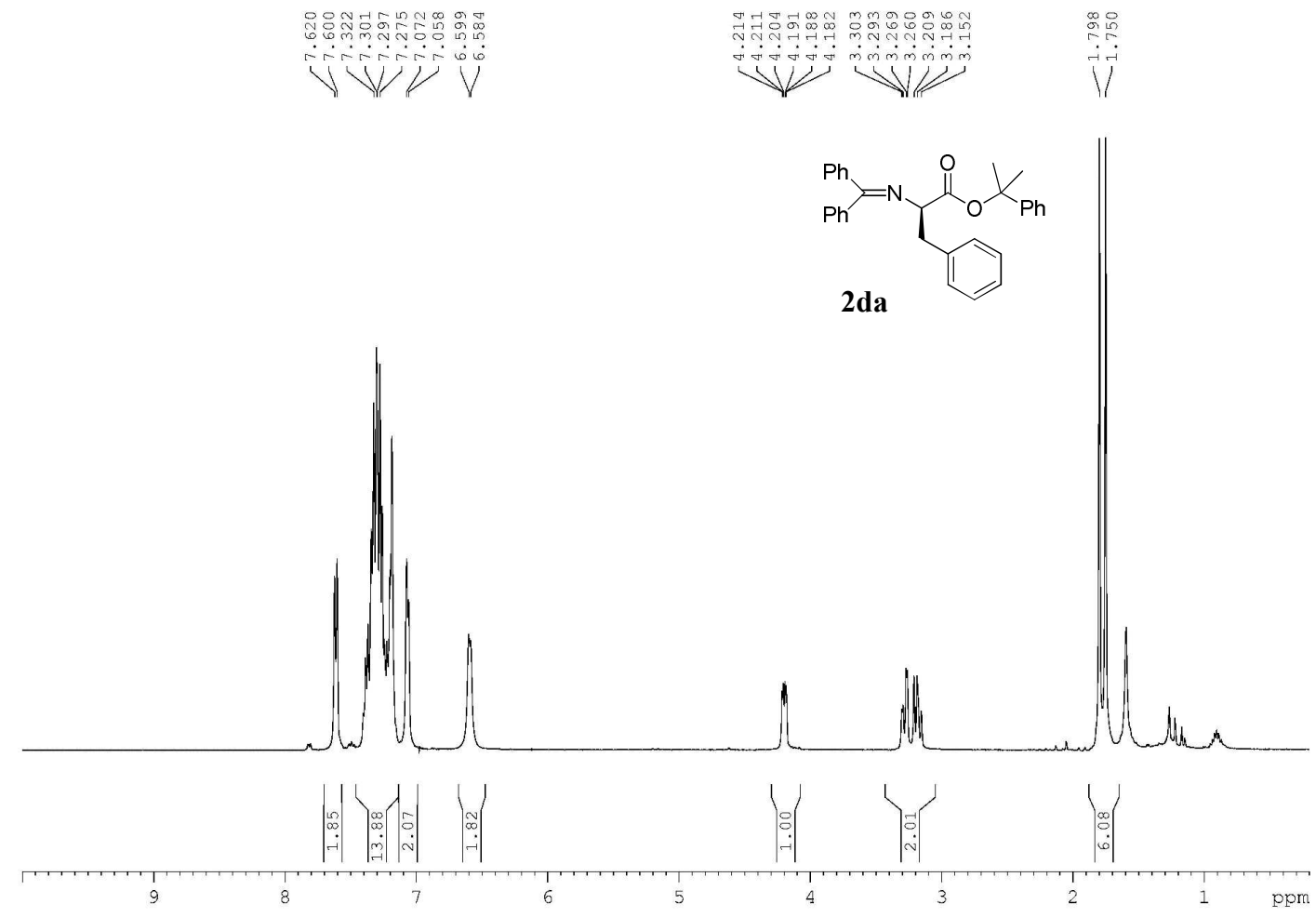

${ }^{13} \mathrm{C}$ NMR of compound 2 da $\left(100 \mathrm{MHz}, \mathrm{CDCl}_{3}\right)$

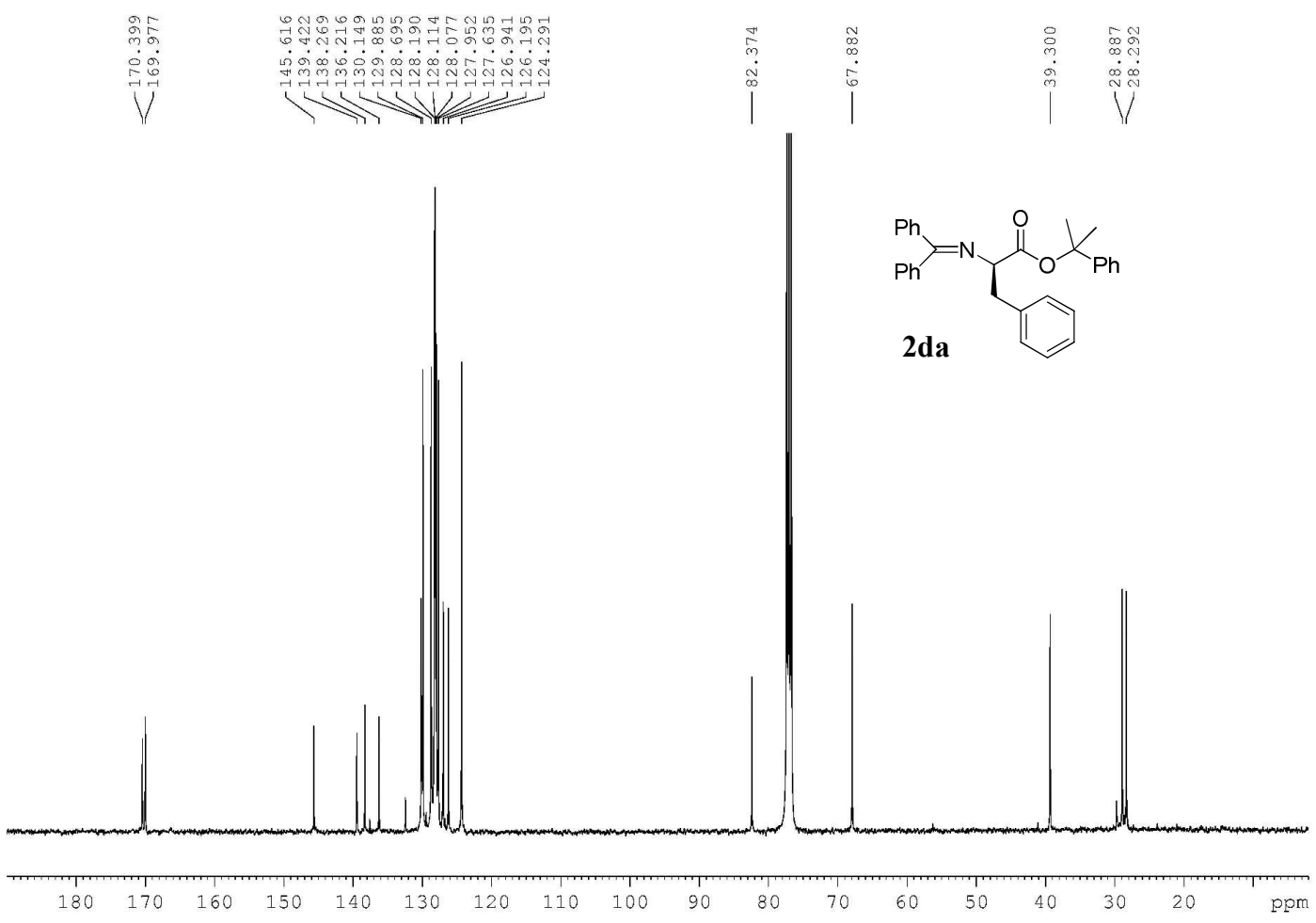


${ }^{1} \mathrm{H}$ NMR of compound $\mathbf{2 d b}\left(400 \mathrm{MHz}, \mathrm{CDCl}_{3}\right.$ )

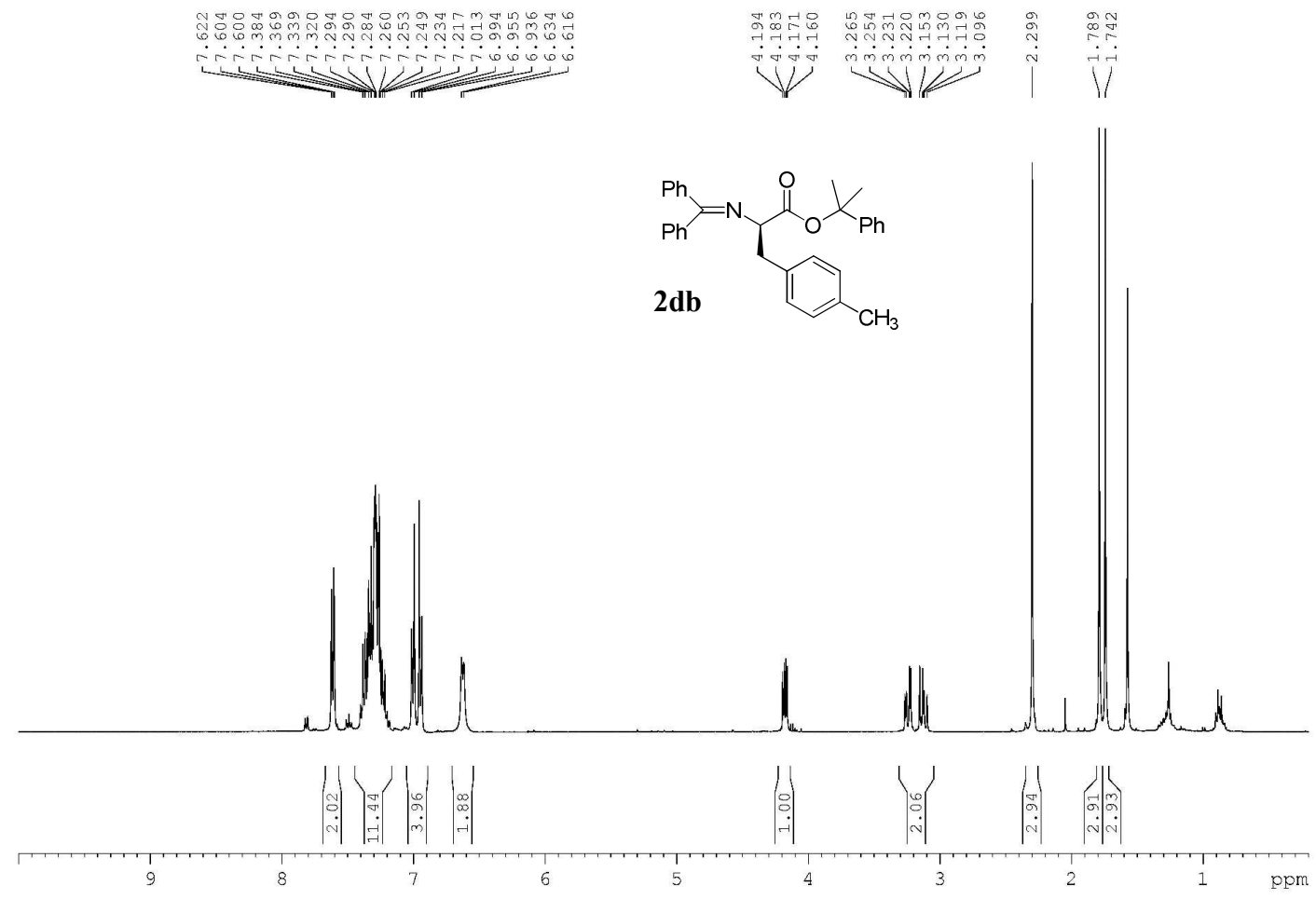

${ }^{13} \mathrm{C}$ NMR of compound $\mathbf{2 d b}\left(100 \mathrm{MHz}, \mathrm{CDCl}_{3}\right)$

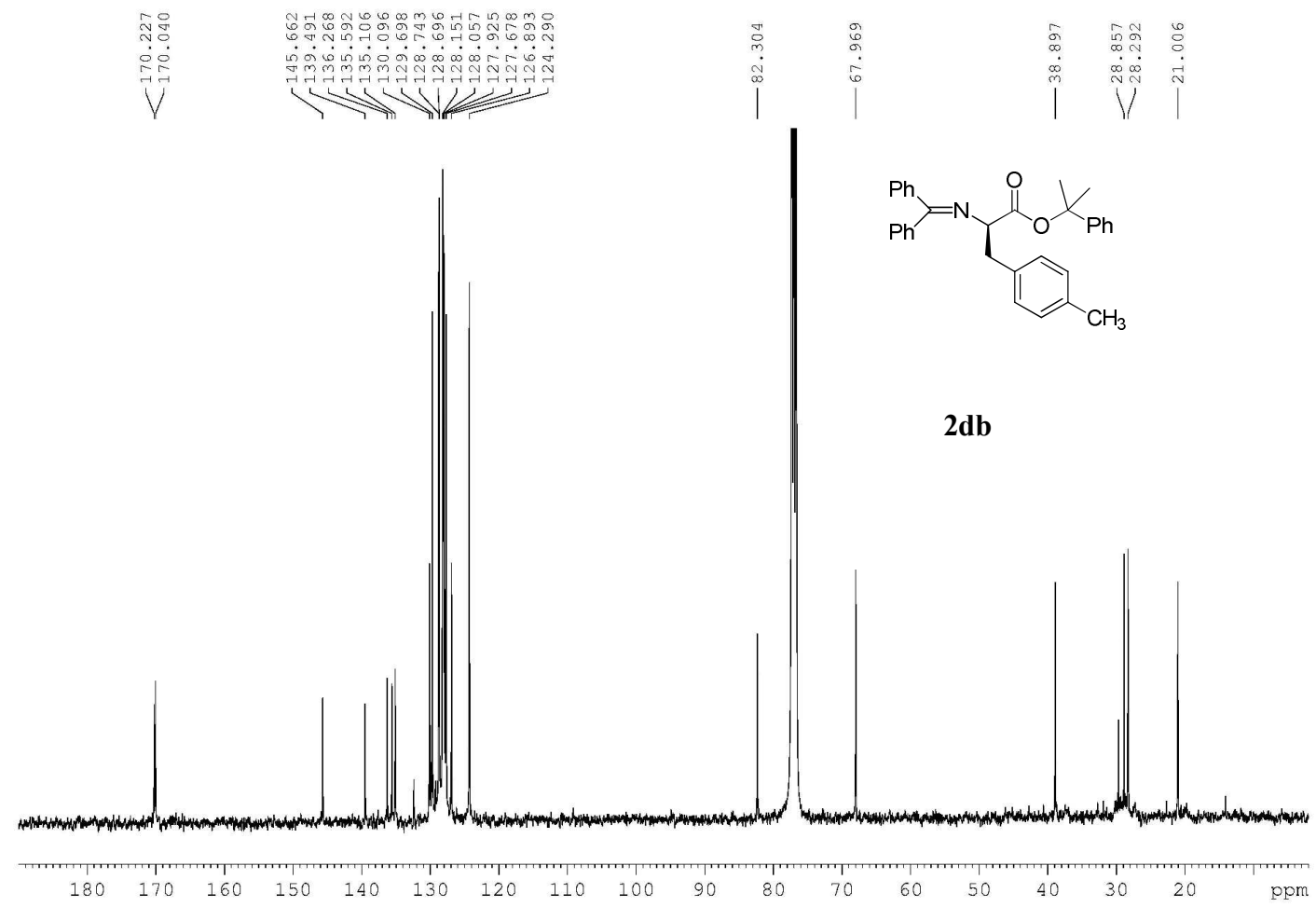


${ }^{1} \mathrm{H}$ NMR of compound $\mathbf{2 d c}\left(400 \mathrm{MHz}, \mathrm{CDCl}_{3}\right)$

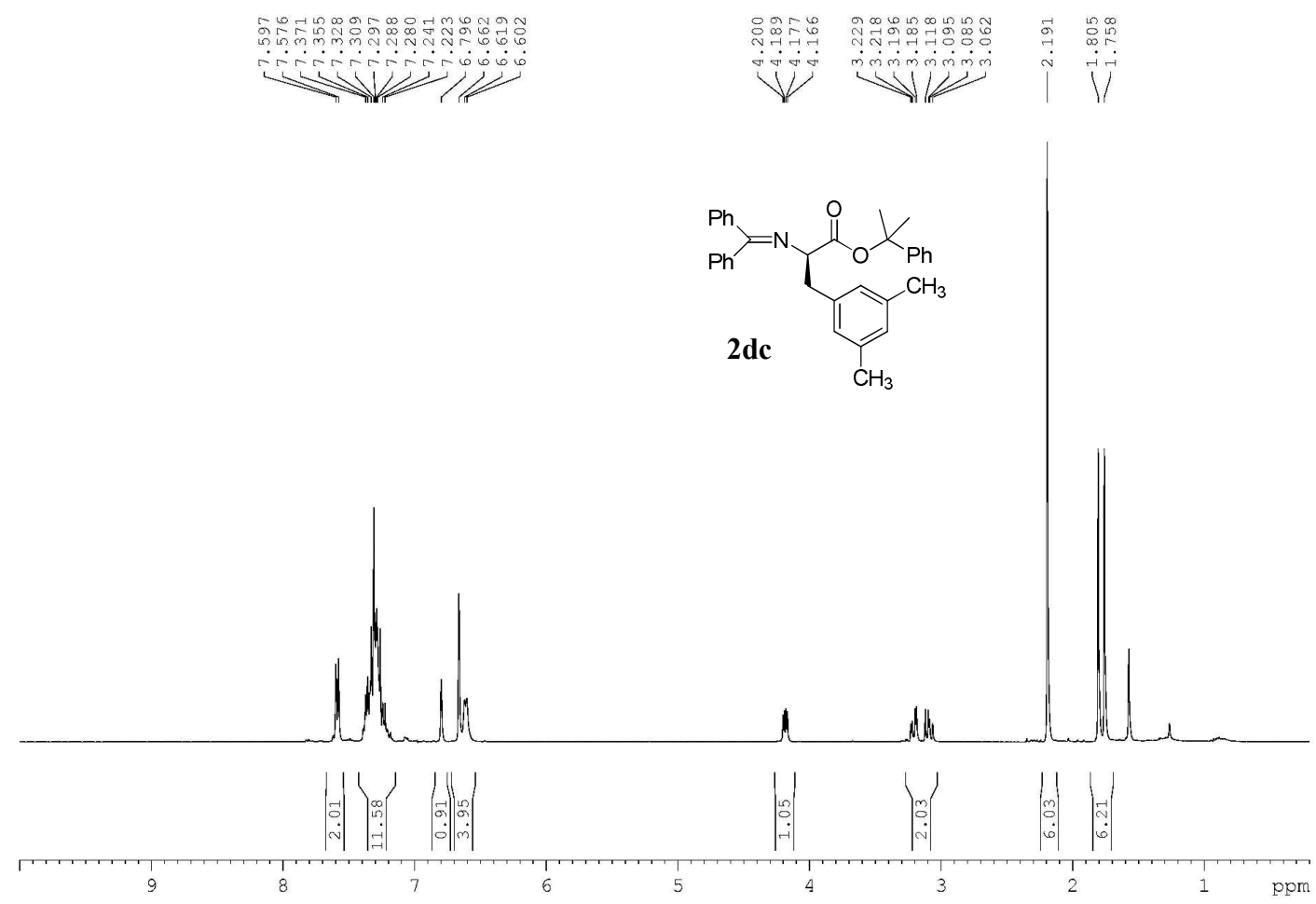

${ }^{13} \mathrm{C}$ NMR of compound $2 \mathbf{d c}\left(100 \mathrm{MHz}, \mathrm{CDCl}_{3}\right)$

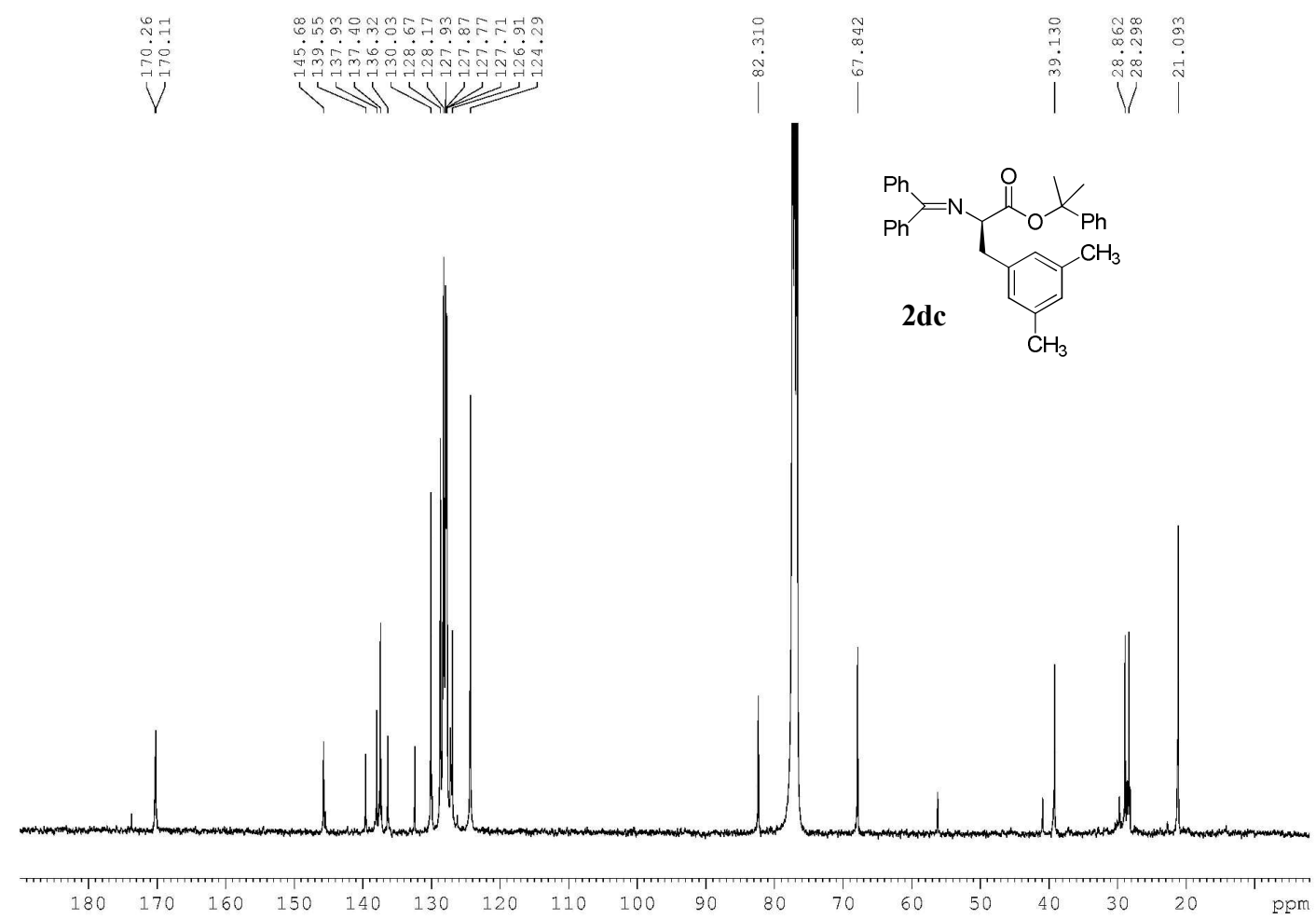


${ }^{1} \mathrm{H}$ NMR of compound $\mathbf{2 d d}\left(400 \mathrm{MHz}, \mathrm{CDCl}_{3}\right)$

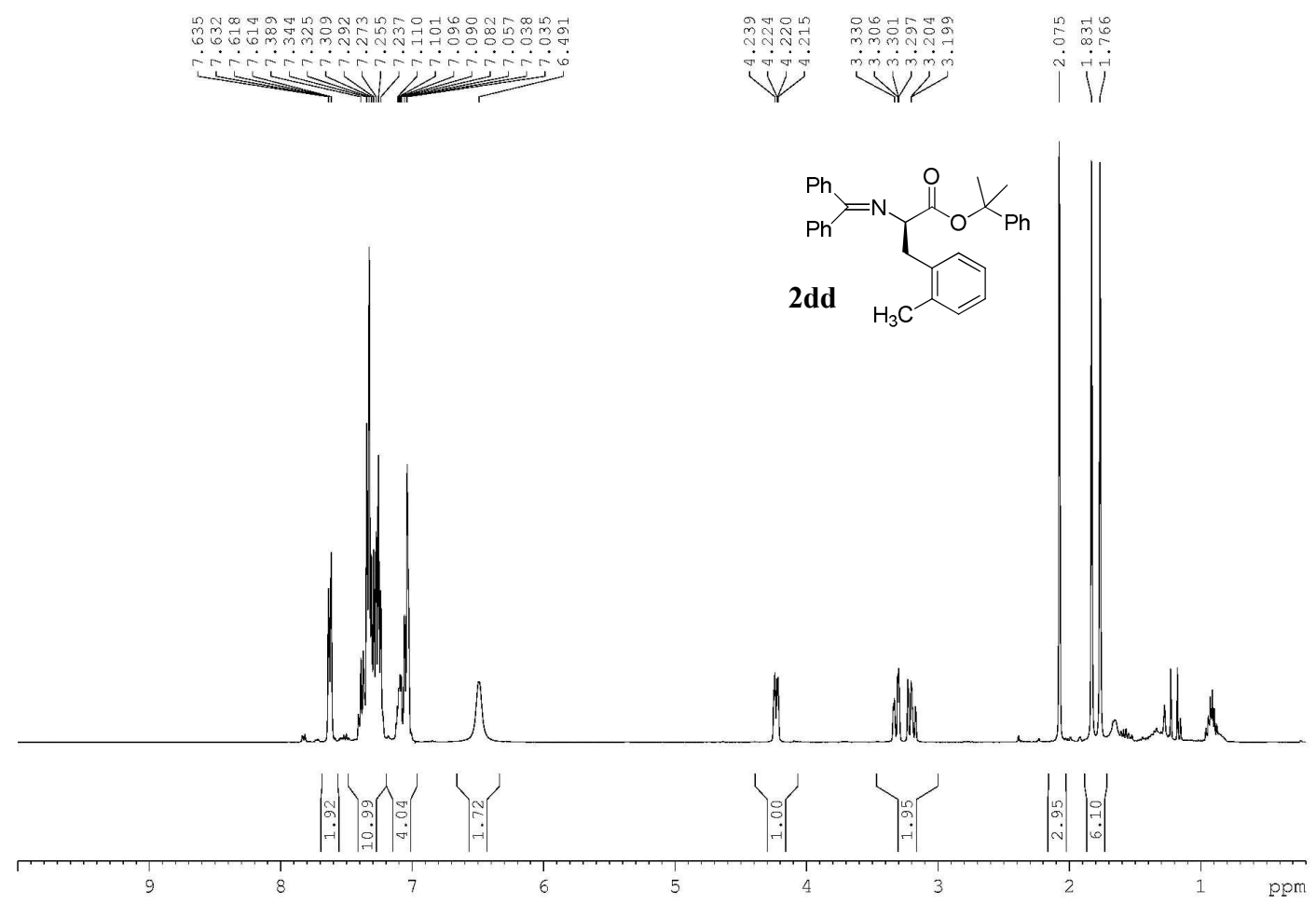

${ }^{13} \mathrm{C}$ NMR of compound $\mathbf{2 d d}\left(100 \mathrm{MHz}, \mathrm{CDCl}_{3}\right)$

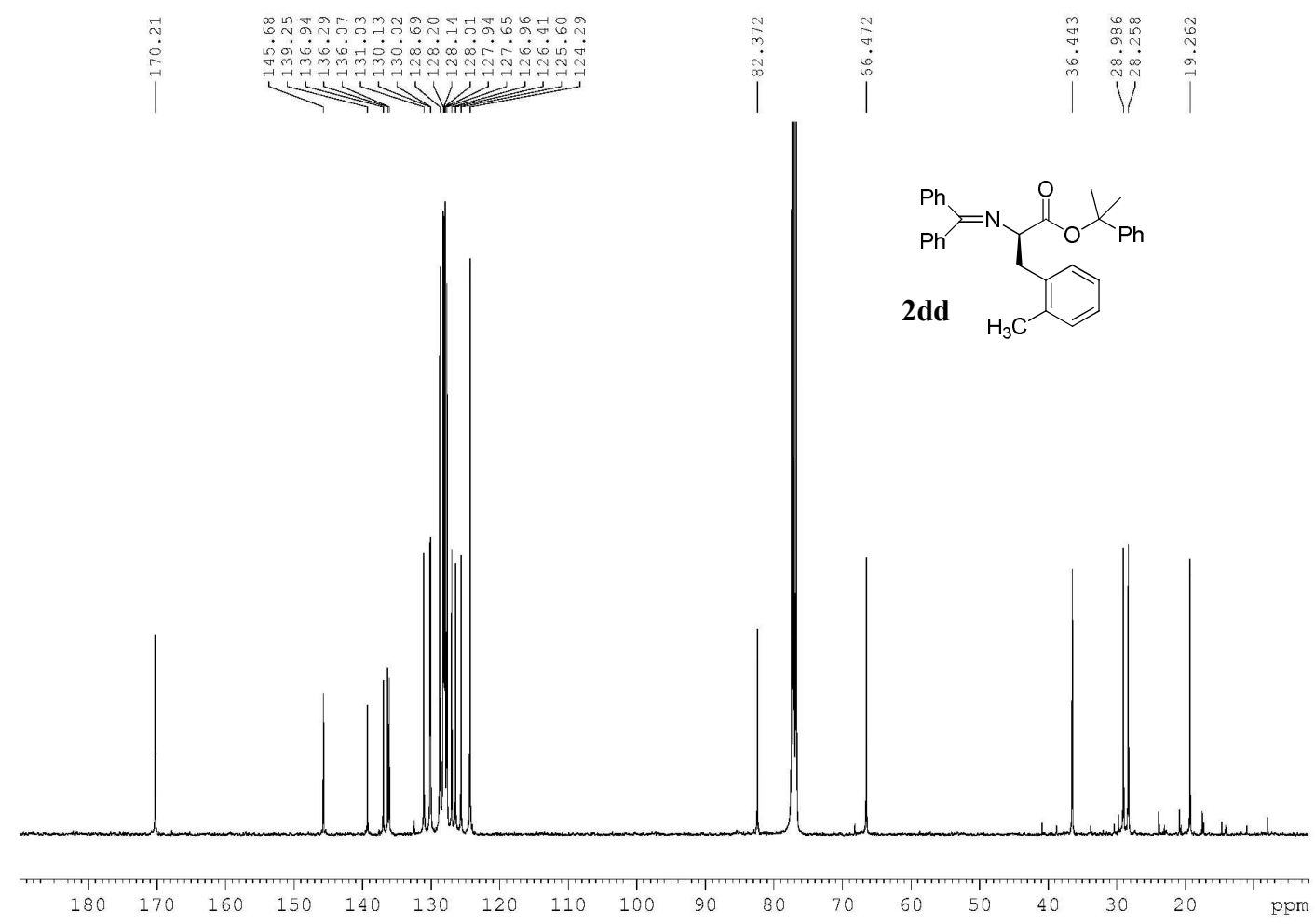


${ }^{1} \mathrm{H}$ NMR of compound 2 de $\left(400 \mathrm{MHz}, \mathrm{CDCl}_{3}\right)$
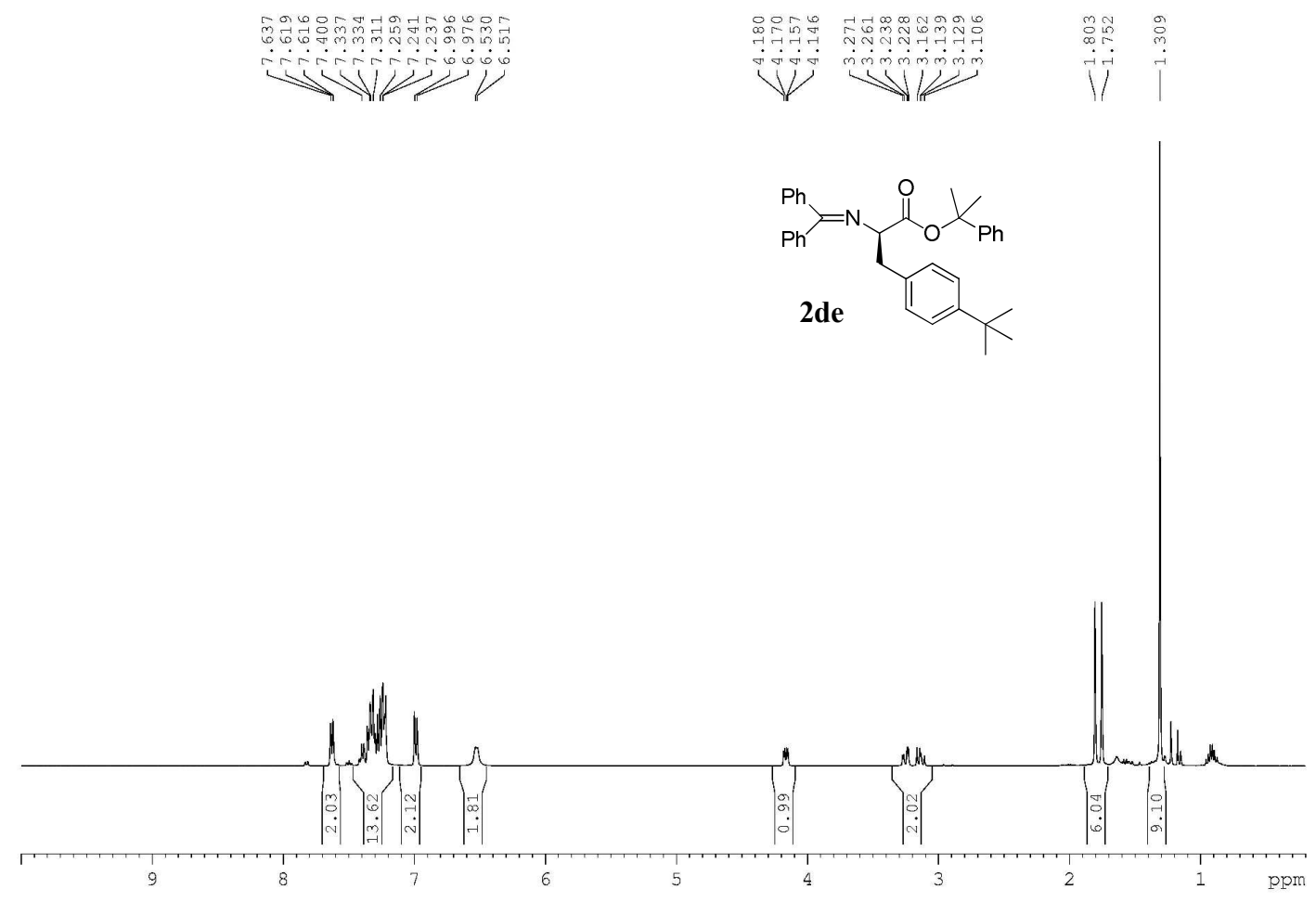

${ }^{13} \mathrm{C}$ NMR of compound 2de (100 MHz, $\left.\mathrm{CDCl}_{3}\right)$

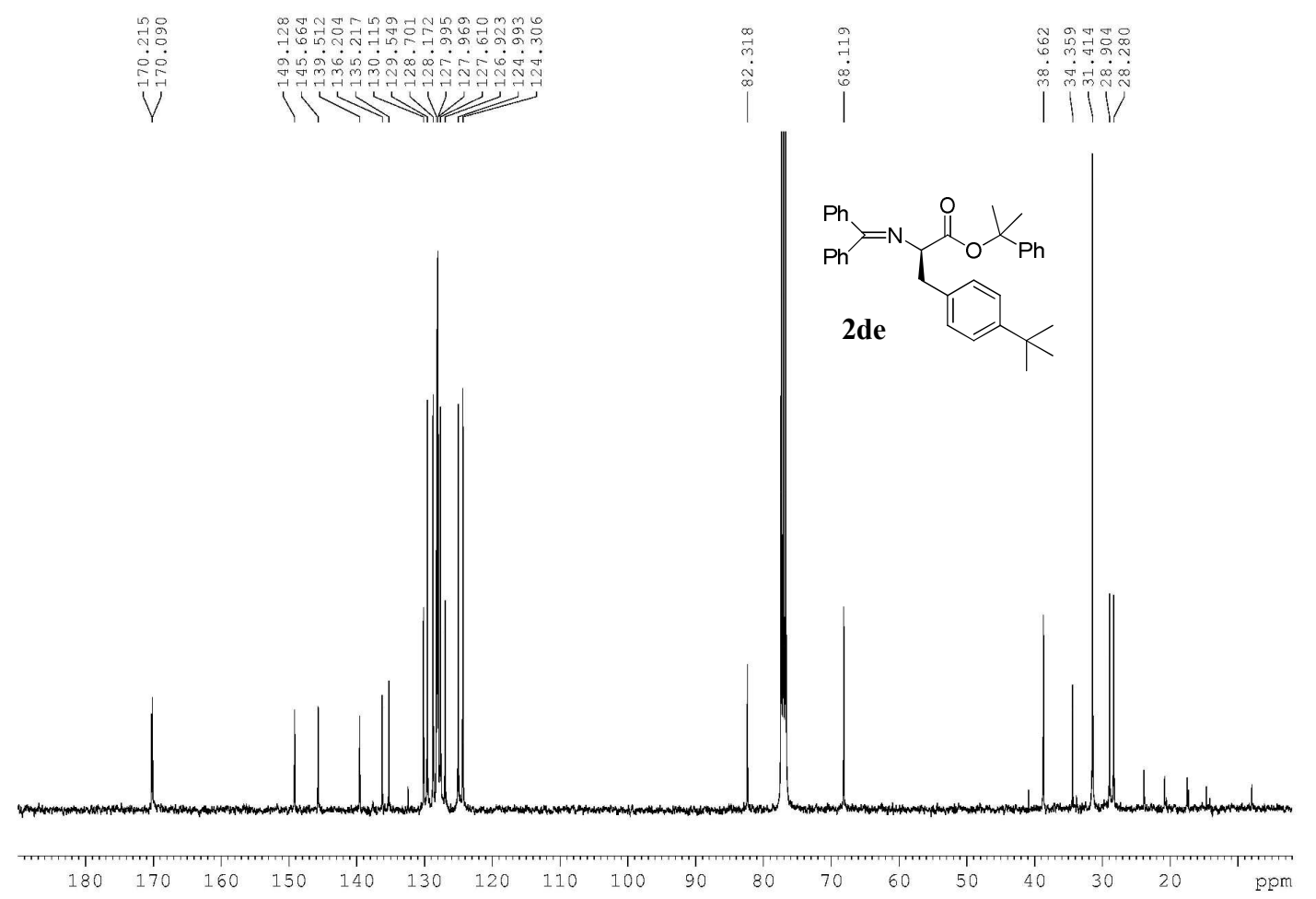


${ }^{1} \mathrm{H}$ NMR of compound $2 \mathbf{d f}\left(400 \mathrm{MHz}, \mathrm{CDCl}_{3}\right)$

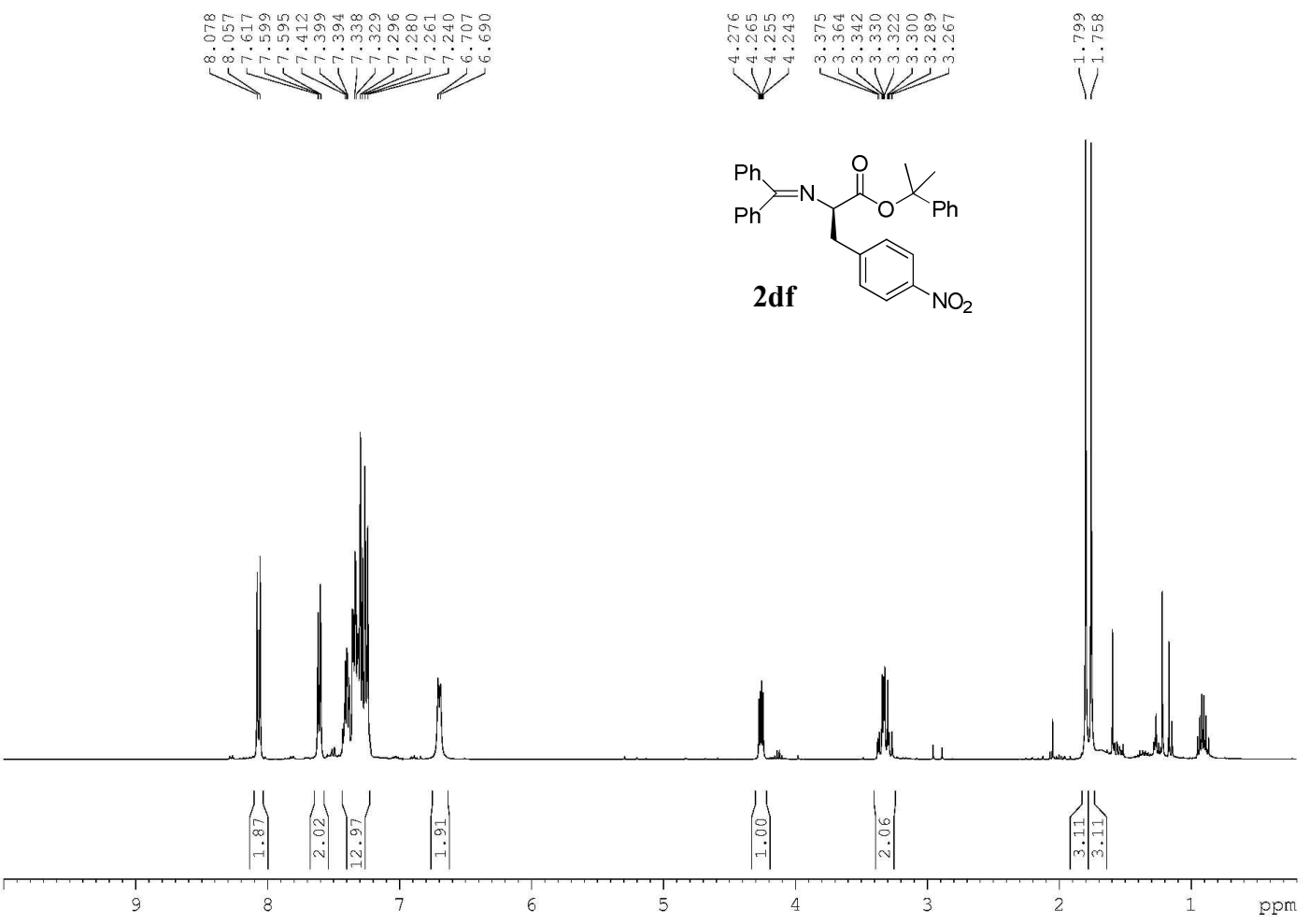

${ }^{13} \mathrm{C}$ NMR of compound $2 \mathbf{d f}\left(100 \mathrm{MHz}, \mathrm{CDCl}_{3}\right)$

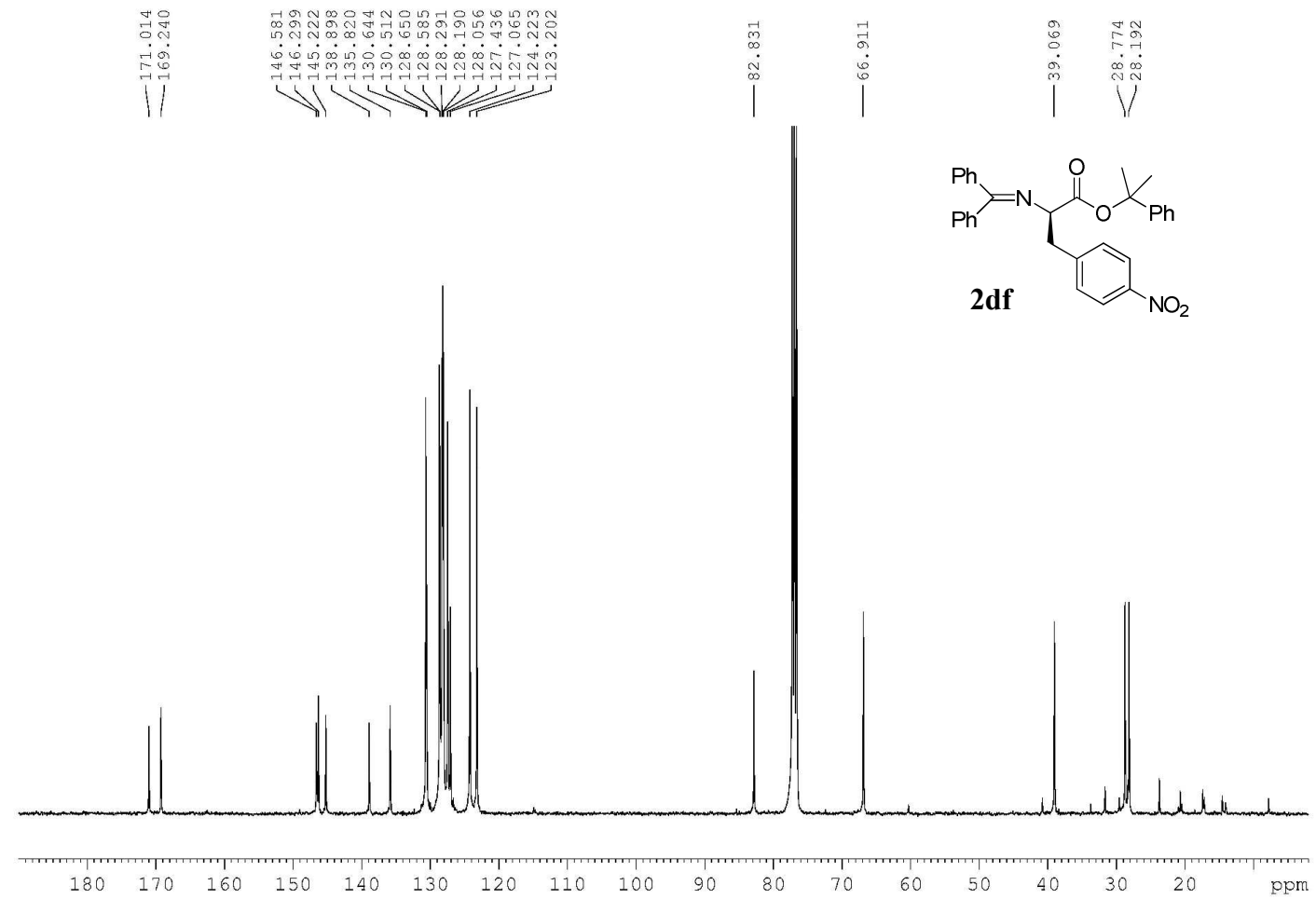


${ }^{1} \mathrm{H}$ NMR of compound $\mathbf{2 d g}\left(400 \mathrm{MHz}, \mathrm{CDCl}_{3}\right)$

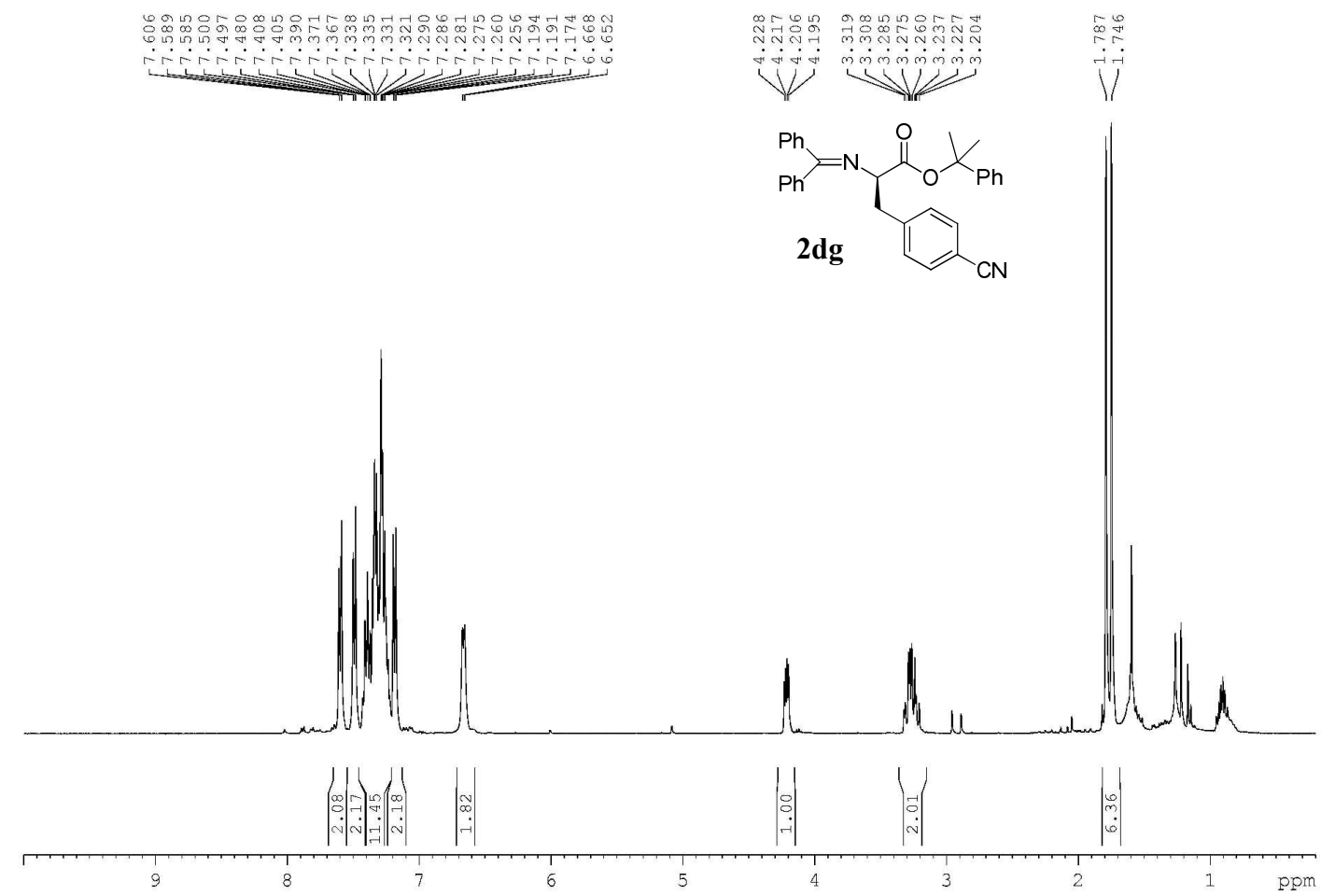

${ }^{13} \mathrm{C}$ NMR of compound $2 \mathbf{d g}\left(100 \mathrm{MHz}, \mathrm{CDCl}_{3}\right)$

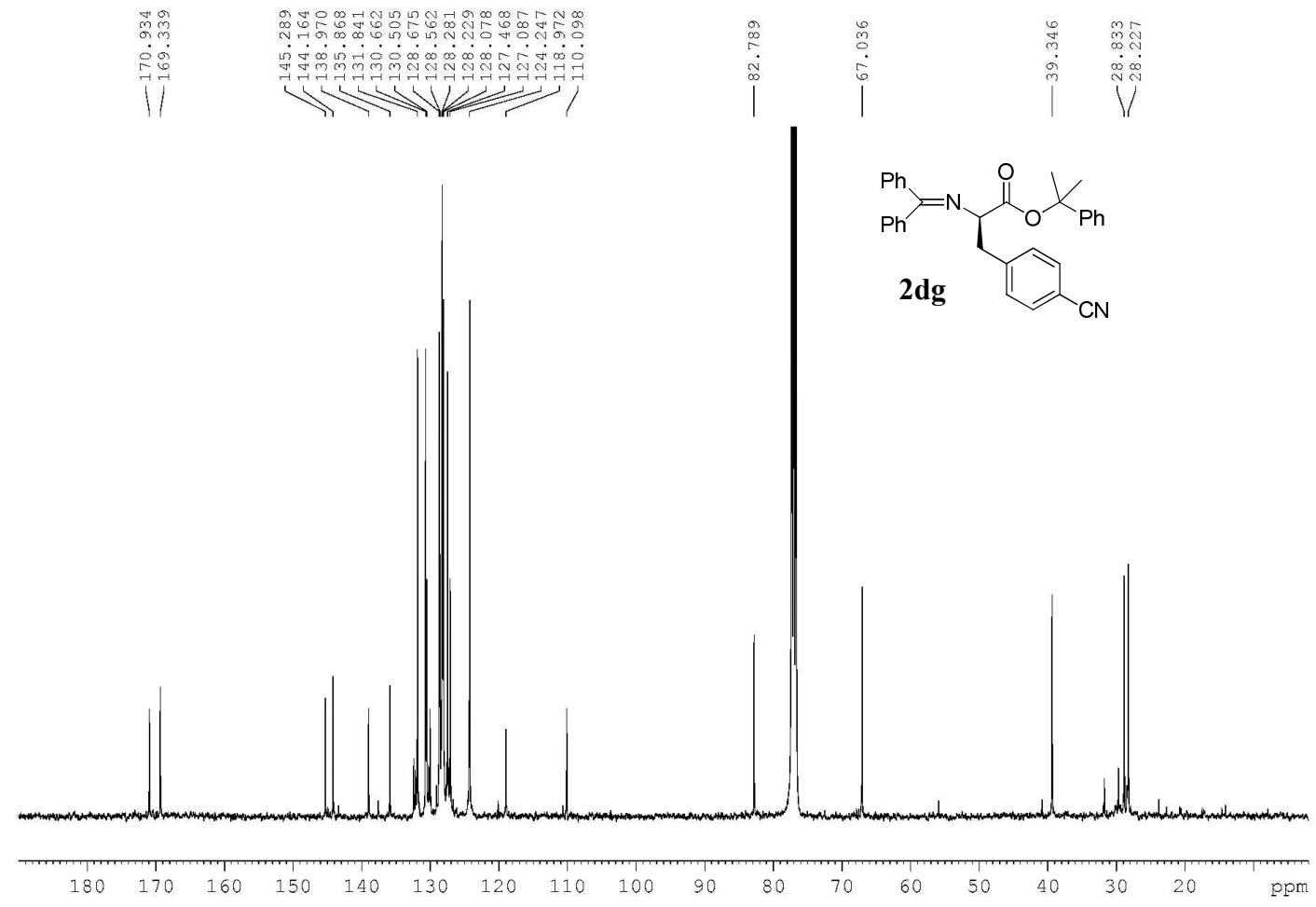


${ }^{1} \mathrm{H}$ NMR of compound $\mathbf{2 d h}\left(400 \mathrm{MHz}, \mathrm{CDCl}_{3}\right)$

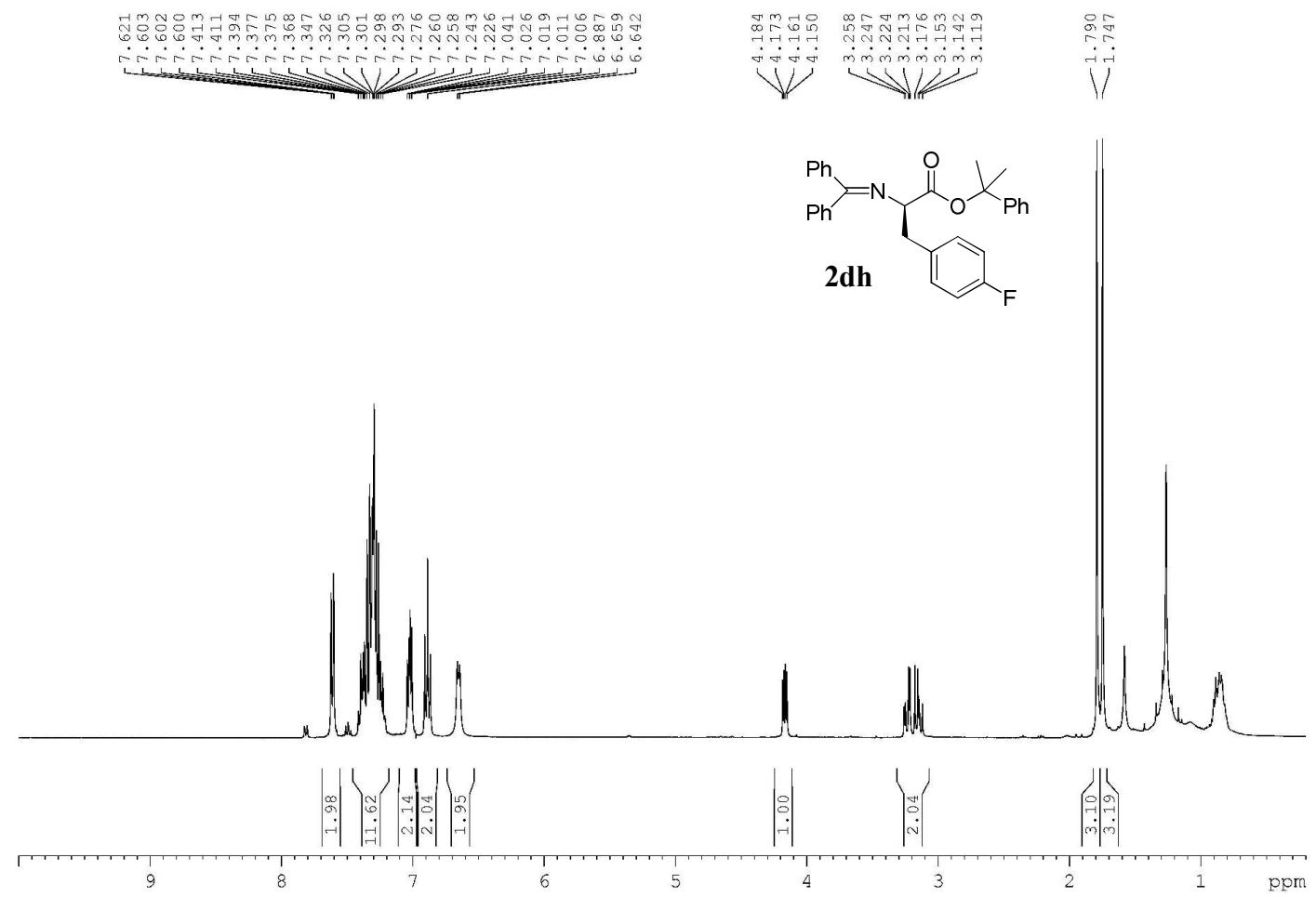

${ }^{13} \mathrm{C}$ NMR of compound $\mathbf{2 d h}\left(100 \mathrm{MHz}, \mathrm{CDCl}_{3}\right)$

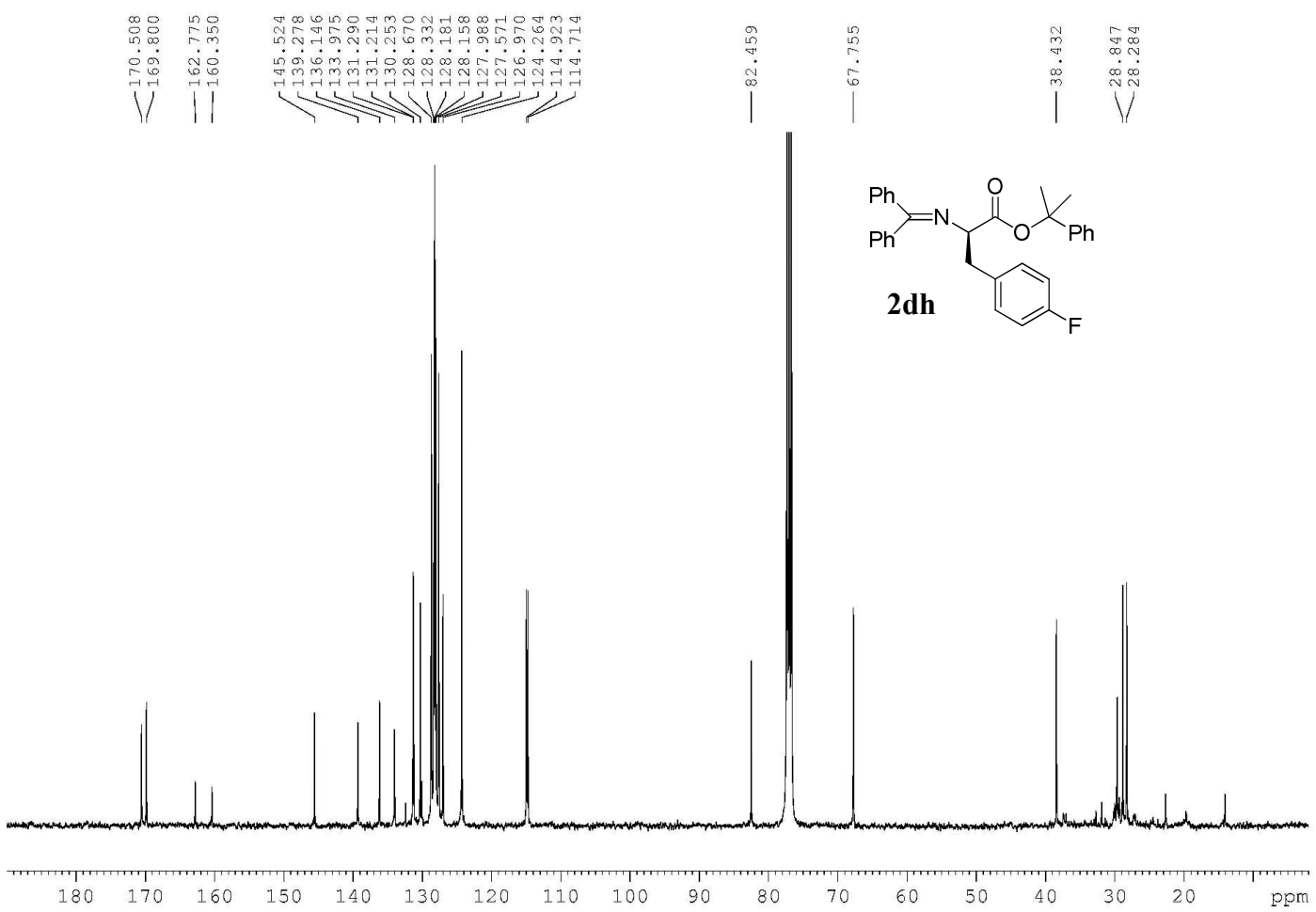


${ }^{1} \mathrm{H}$ NMR of compound 2di (400 MHz, $\mathrm{CDCl}_{3}$ )

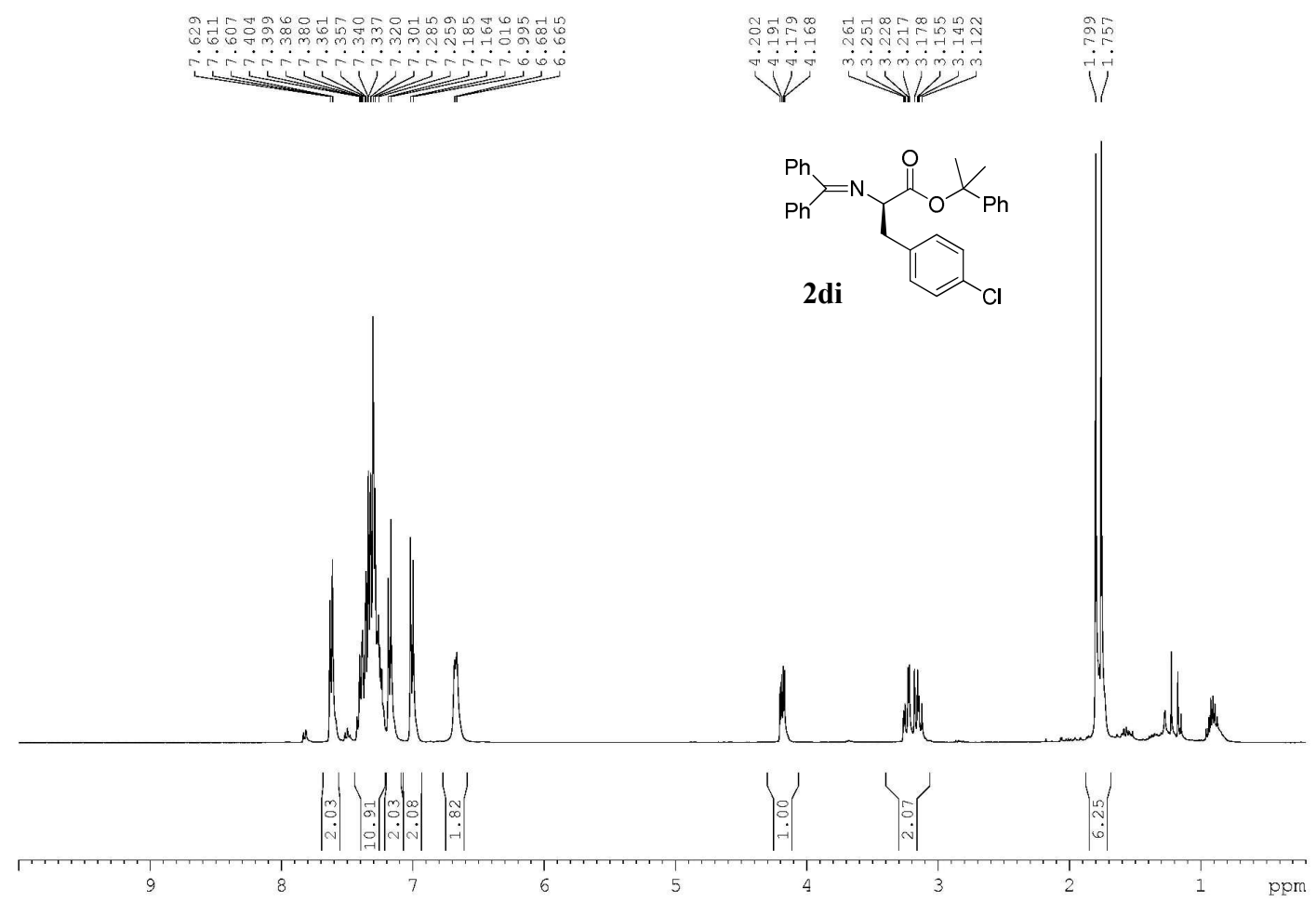

${ }^{13} \mathrm{C}$ NMR of compound $2 \mathbf{d i}\left(100 \mathrm{MHz}, \mathrm{CDCl}_{3}\right)$

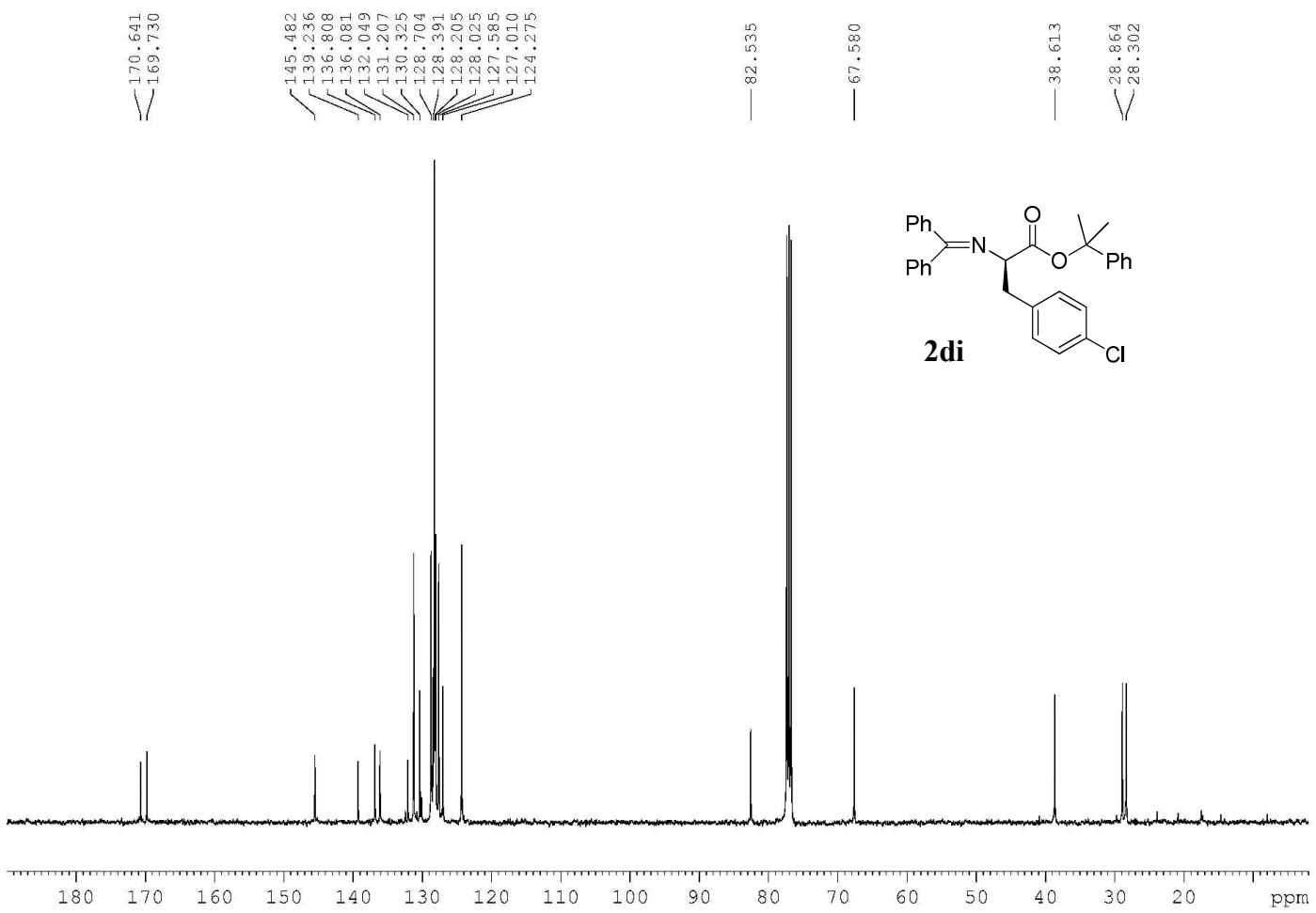


${ }^{1} \mathrm{H}$ NMR of compound $\mathbf{2 d j}\left(400 \mathrm{MHz}, \mathrm{CDCl}_{3}\right.$ )

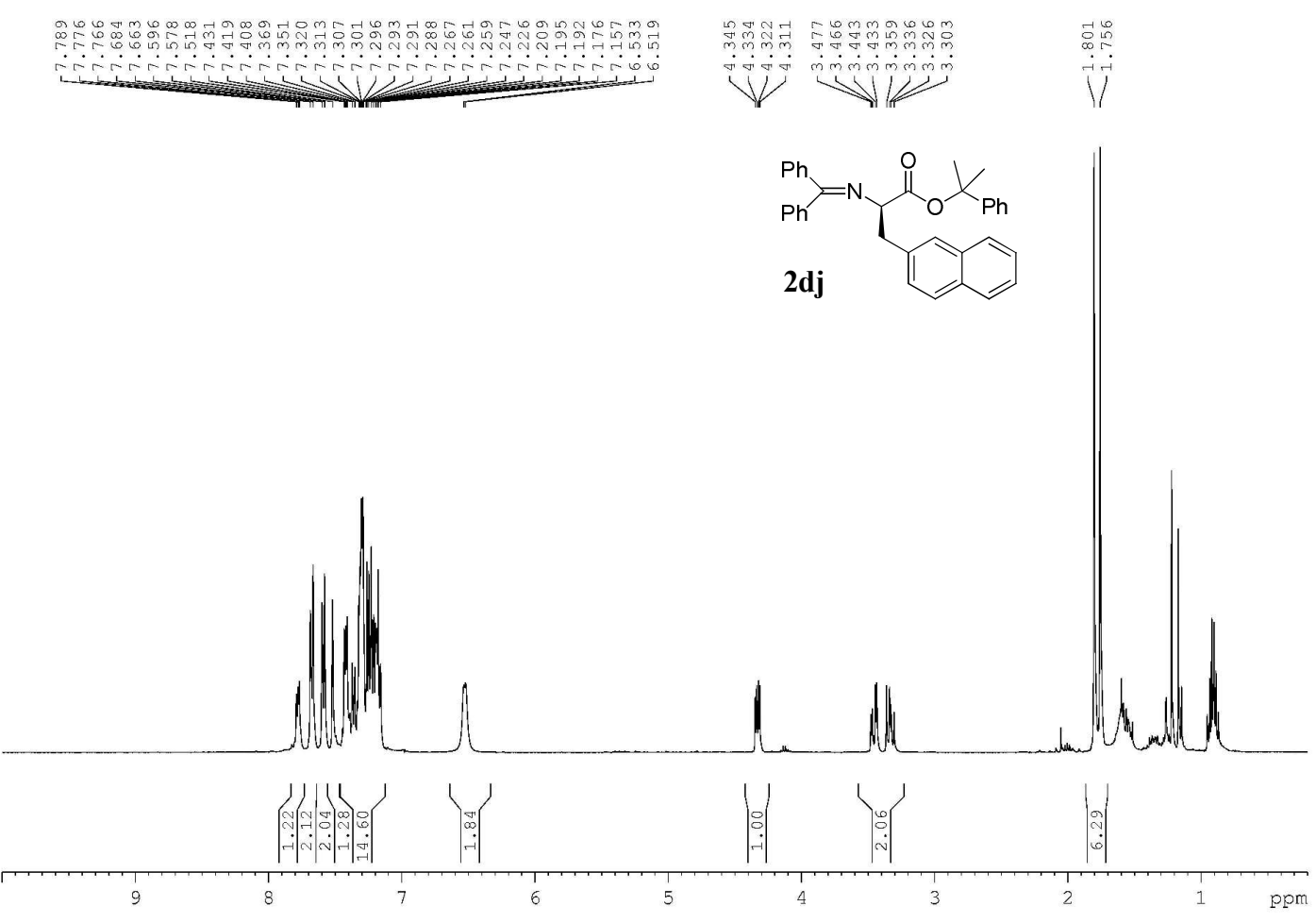

${ }^{13} \mathrm{C}$ NMR of compound $\mathbf{2 d j}\left(100 \mathrm{MHz}, \mathrm{CDCl}_{3}\right)$

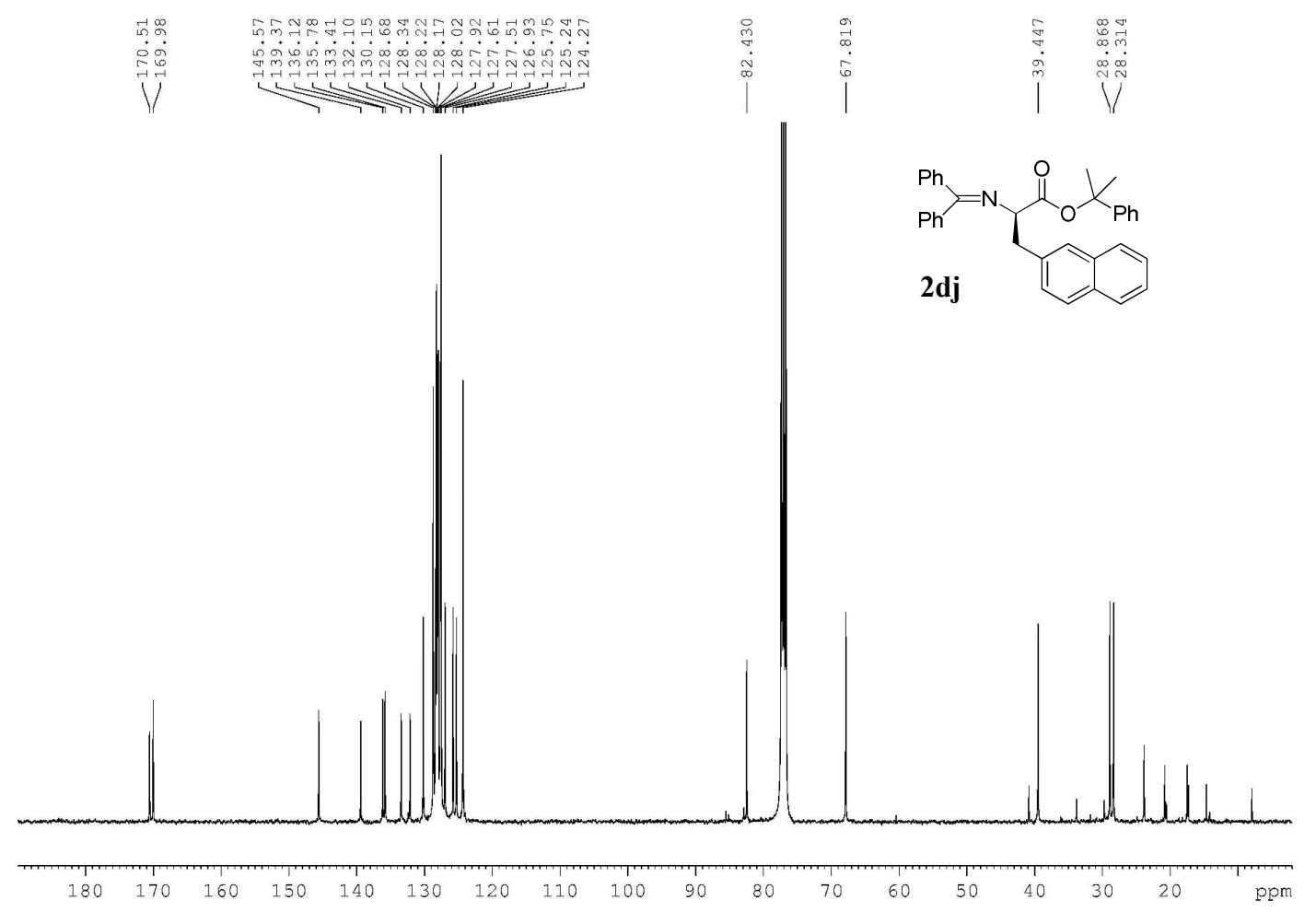


${ }^{1} \mathrm{H}$ NMR of compound $2 \mathbf{d k}\left(400 \mathrm{MHz}, \mathrm{CDCl}_{3}\right.$ )

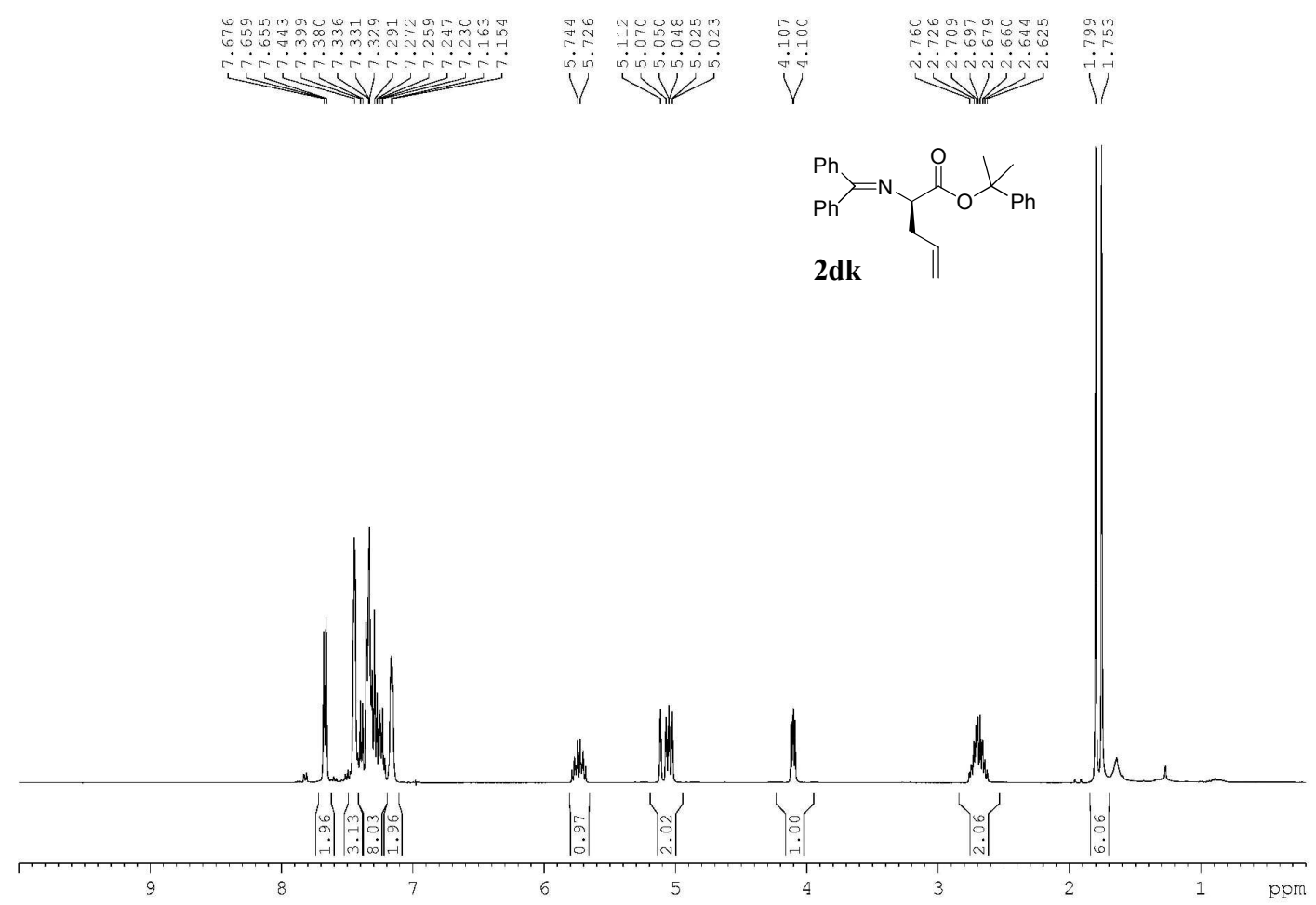

${ }^{13} \mathrm{C}$ NMR of compound 2dk (100 MHz, $\left.\mathrm{CDCl}_{3}\right)$

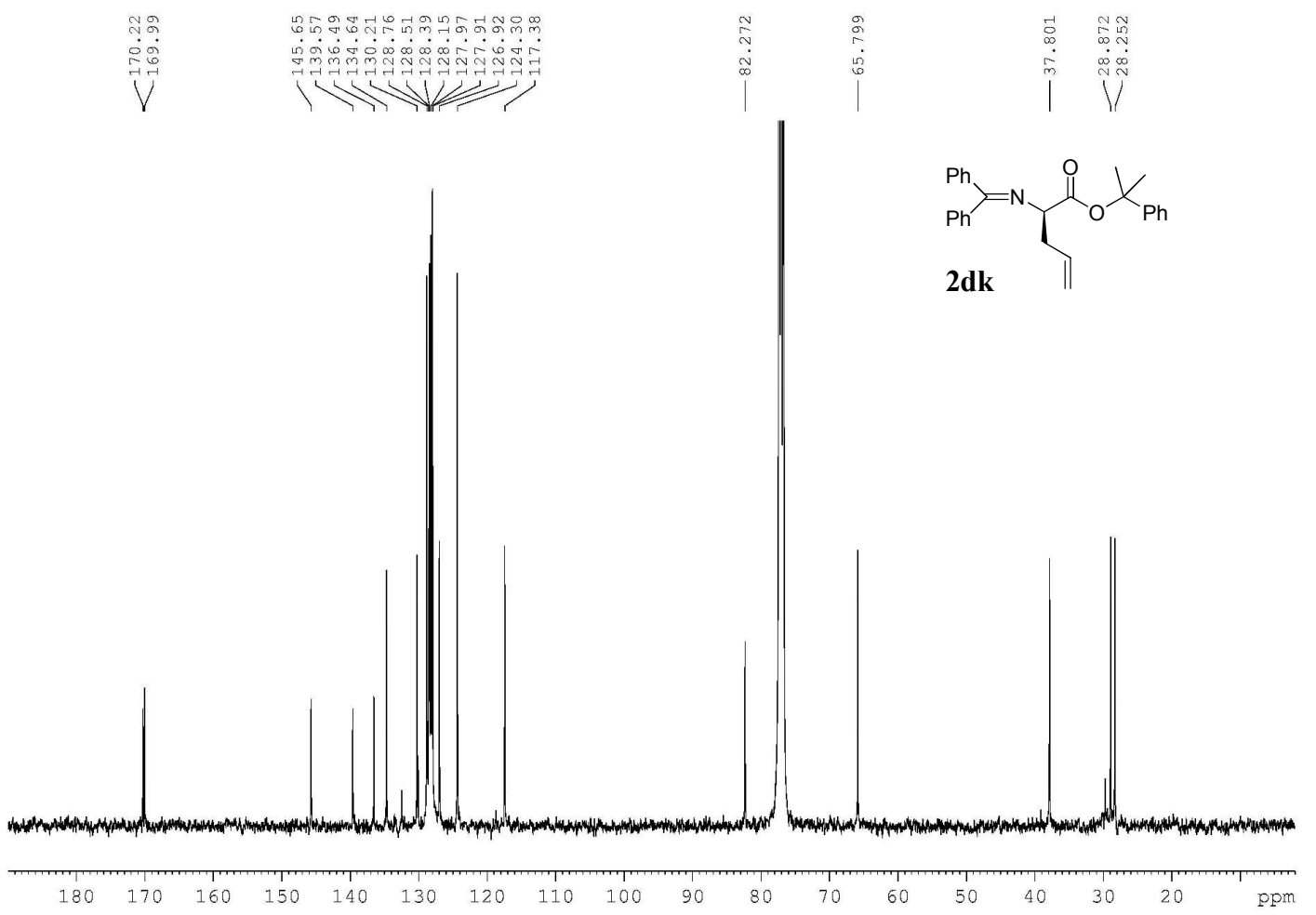


${ }^{1} \mathrm{H}$ NMR of compound $\mathbf{2 d l}\left(400 \mathrm{MHz}, \mathrm{CDCl}_{3}\right)$

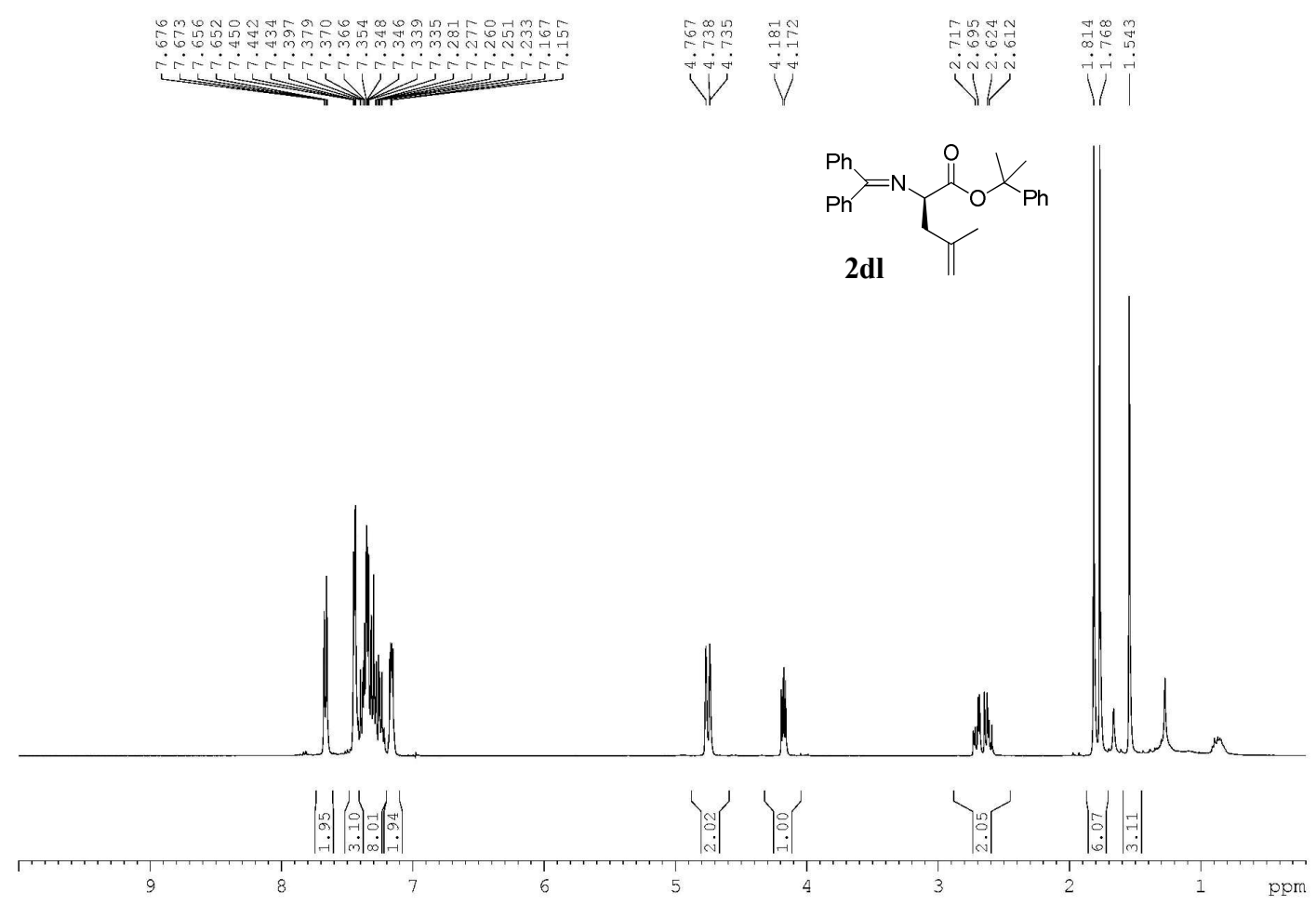

${ }^{13} \mathrm{C}$ NMR of compound $2 \mathbf{d l}\left(100 \mathrm{MHz}, \mathrm{CDCl}_{3}\right)$

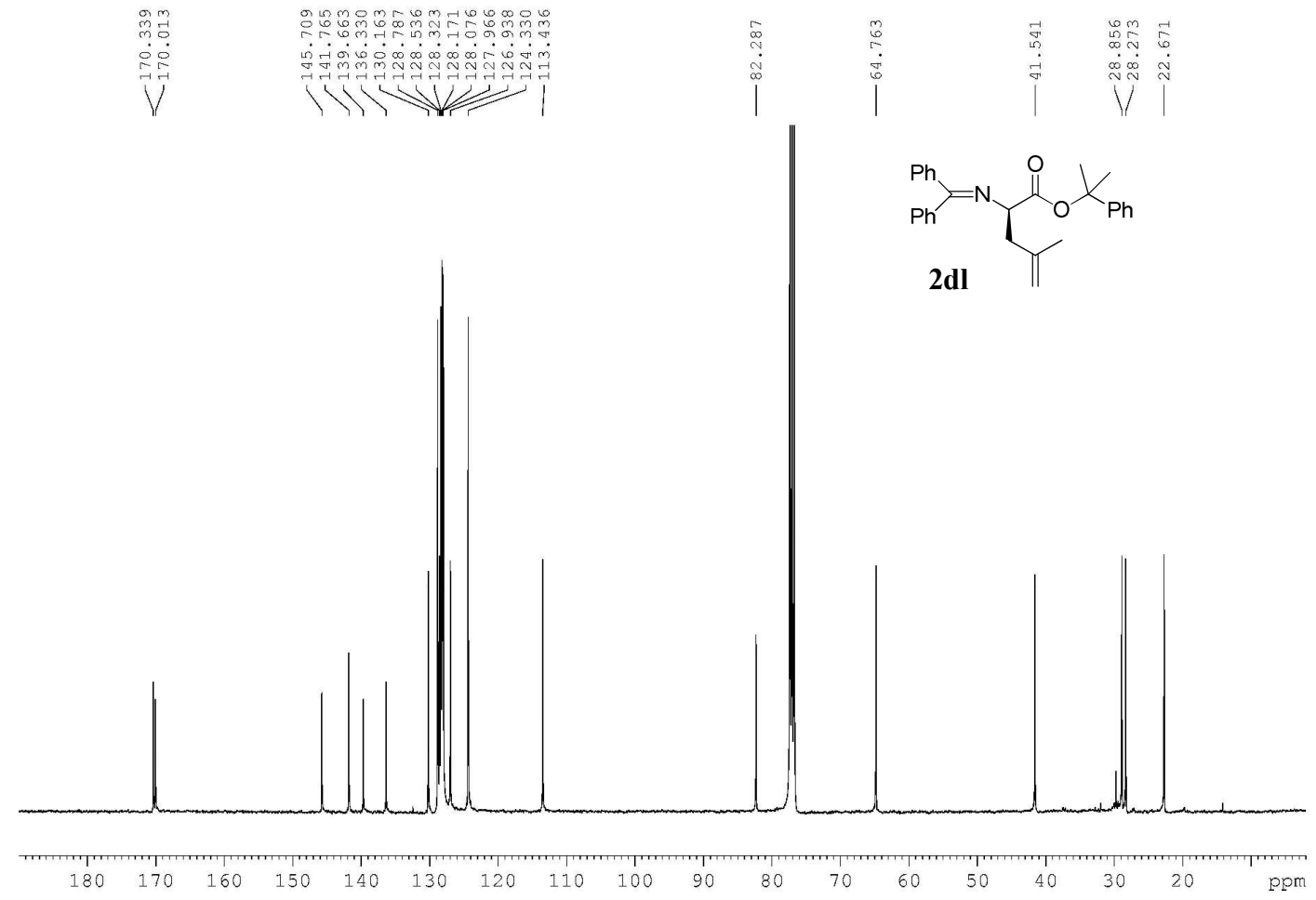


${ }^{1} \mathrm{H}$ NMR of compound $\mathbf{2 d m}\left(400 \mathrm{MHz}, \mathrm{CDCl}_{3}\right)$

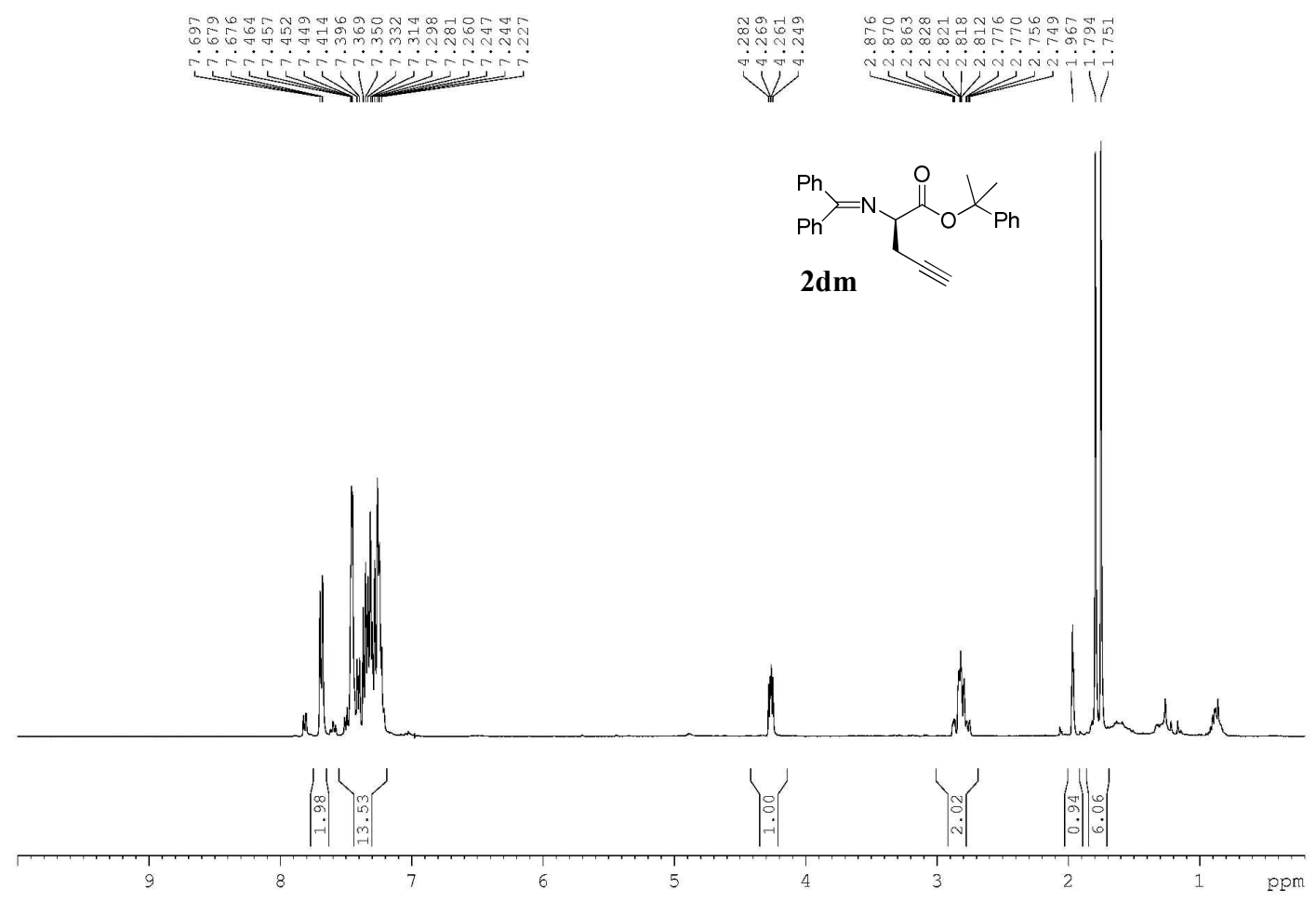

${ }^{13} \mathrm{C}$ NMR of compound $\mathbf{2} \mathbf{d m}\left(100 \mathrm{MHz}, \mathrm{CDCl}_{3}\right)$

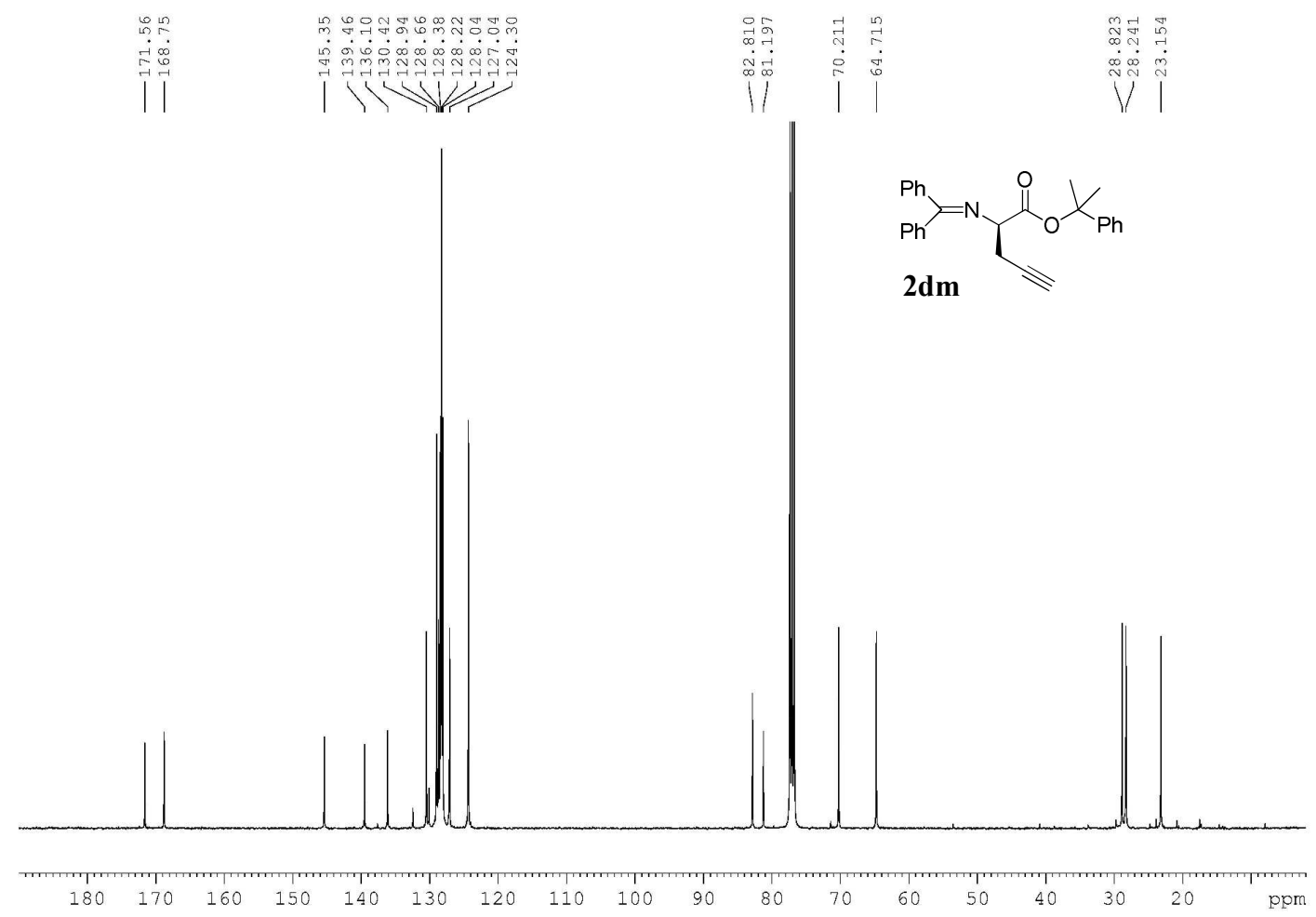


${ }^{1} \mathrm{H}$ NMR of compound $\mathbf{2 d n}\left(400 \mathrm{MHz}, \mathrm{CDCl}_{3}\right)$

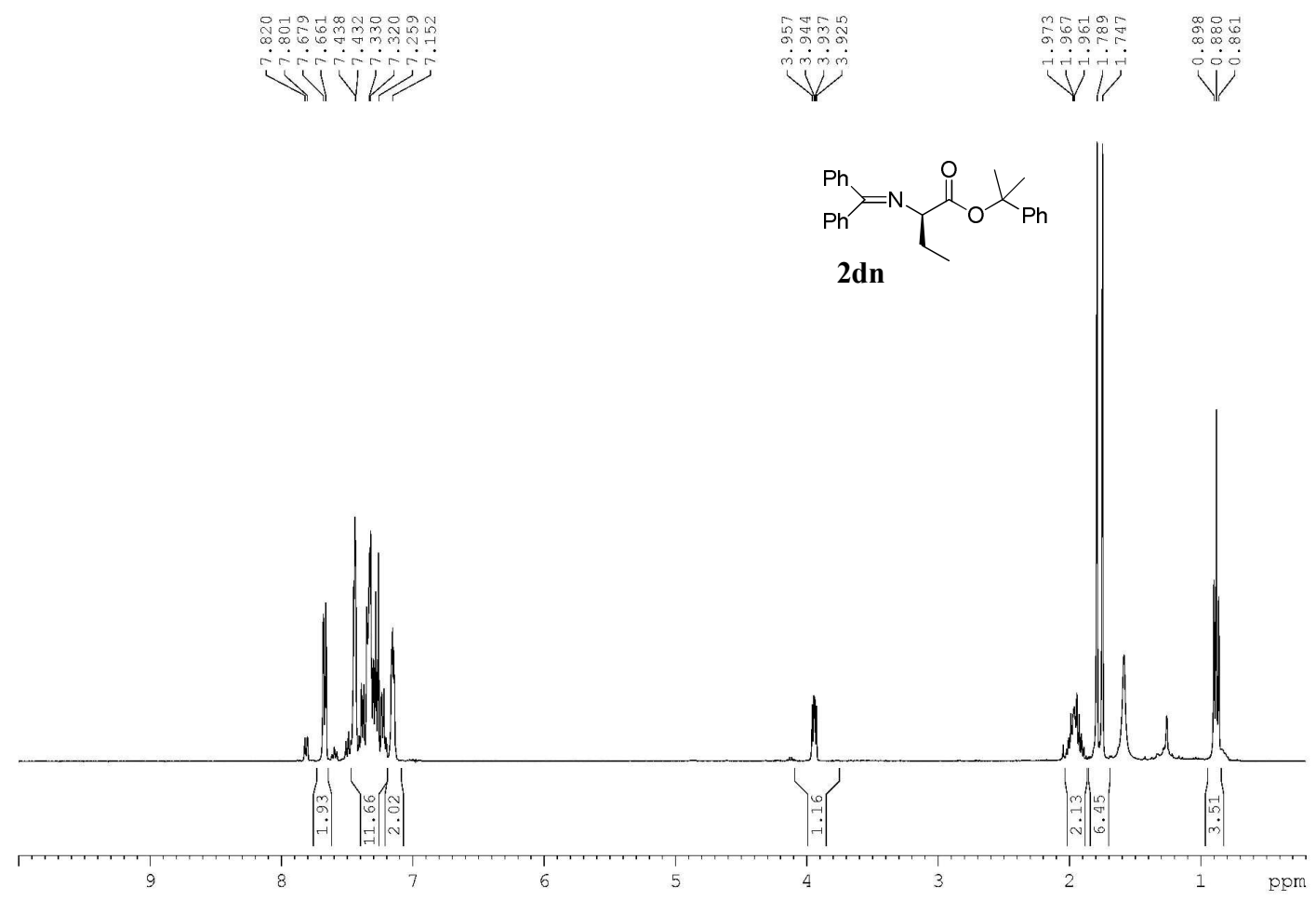

${ }^{13} \mathrm{C}$ NMR of compound $2 \mathbf{d n}\left(100 \mathrm{MHz}, \mathrm{CDCl}_{3}\right)$

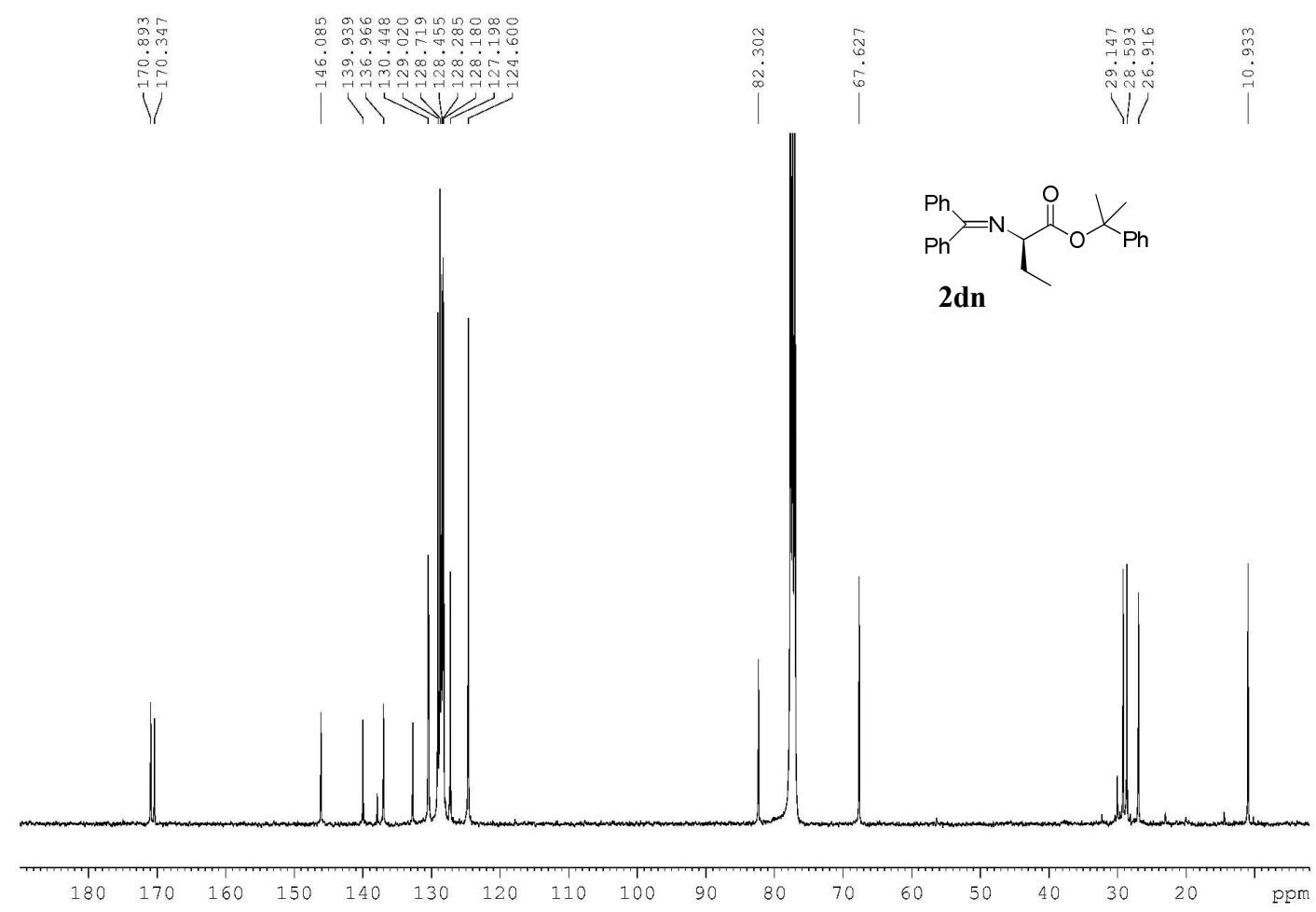


${ }^{1} \mathrm{H}$ NMR of compound $2 \mathbf{d o}\left(600 \mathrm{MHz}, \mathrm{CDCl}_{3}\right)$

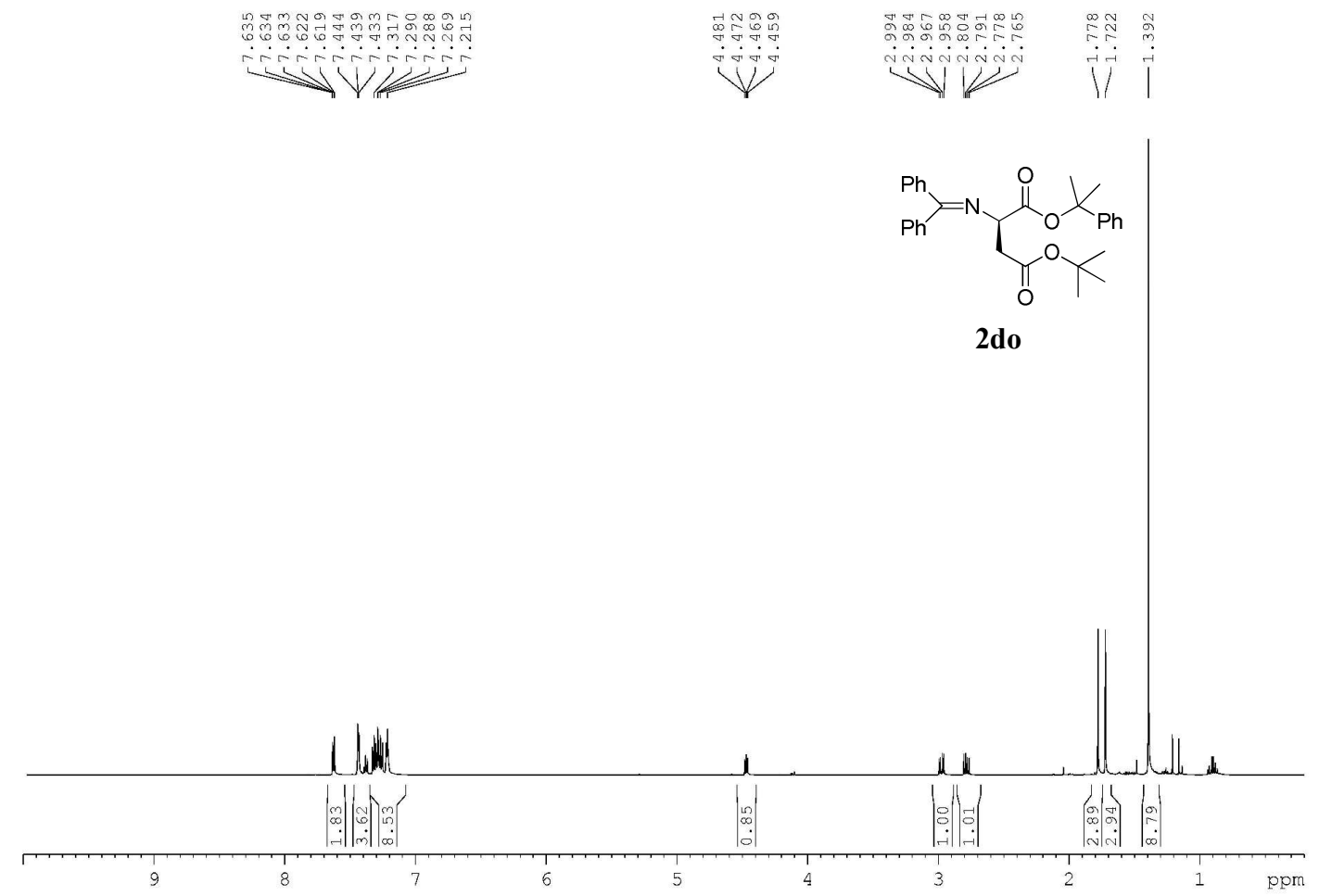

${ }^{13} \mathrm{C}$ NMR of compound $2 \mathbf{d n}\left(150 \mathrm{MHz}, \mathrm{CDCl}_{3}\right)$

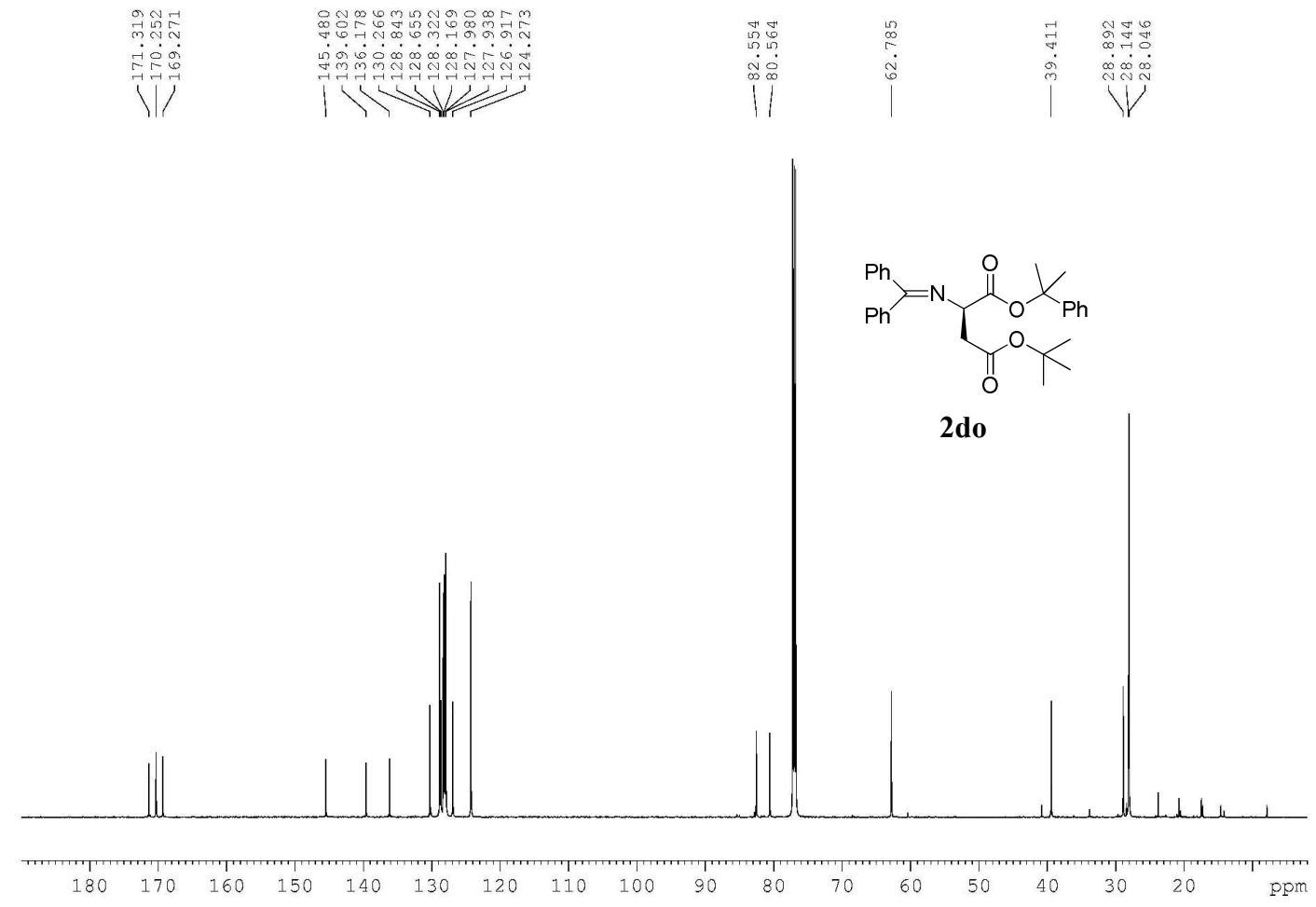




\section{Chiral HPLC traces of compounds 2da-2do}

HPLC chromatograms of compound rac-2da and compound 2da

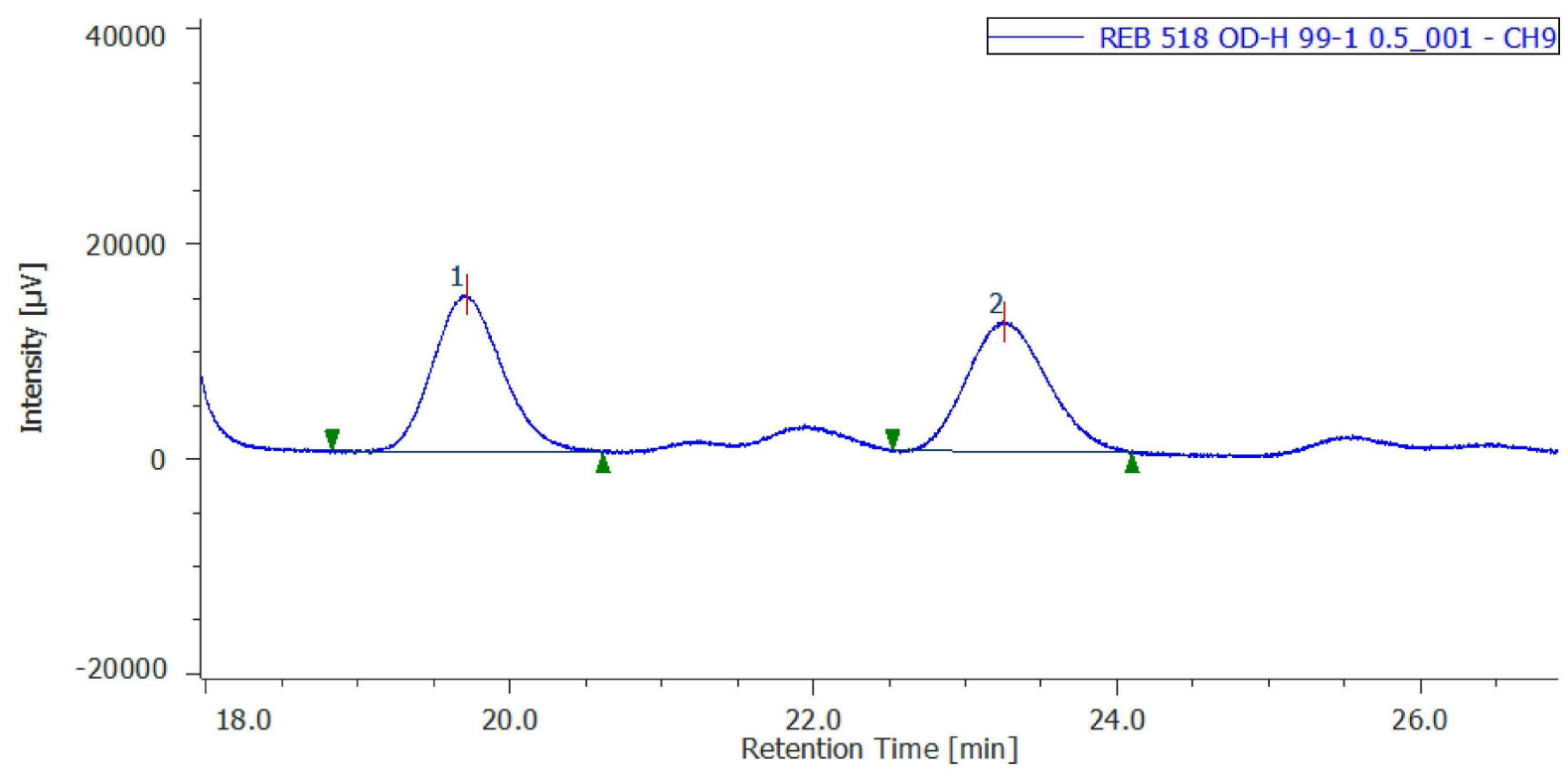

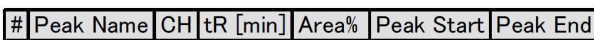

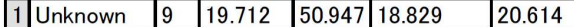

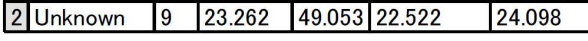

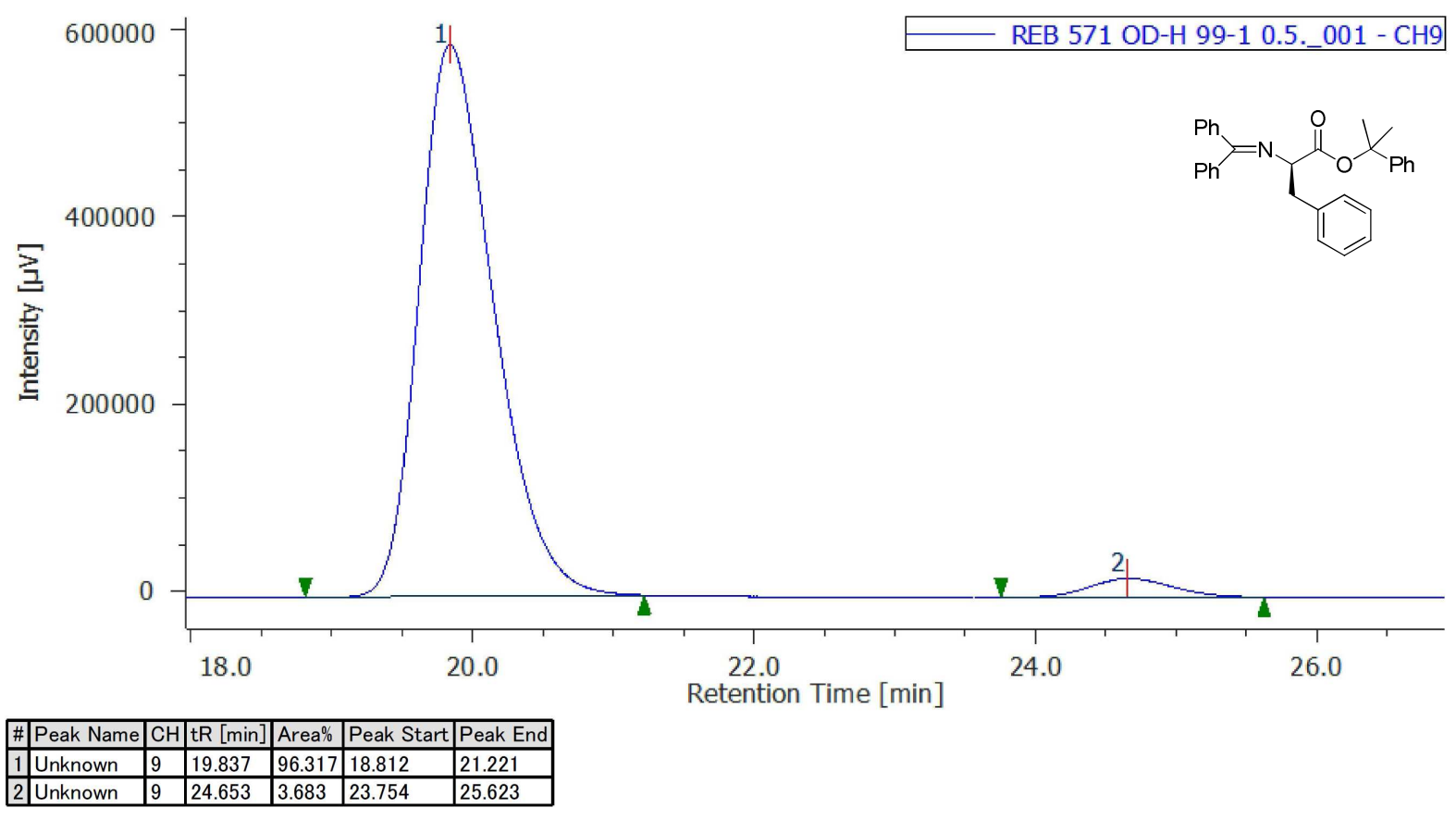


HPLC chromatograms of compound rac-2db and compound $\mathbf{2 d b}$

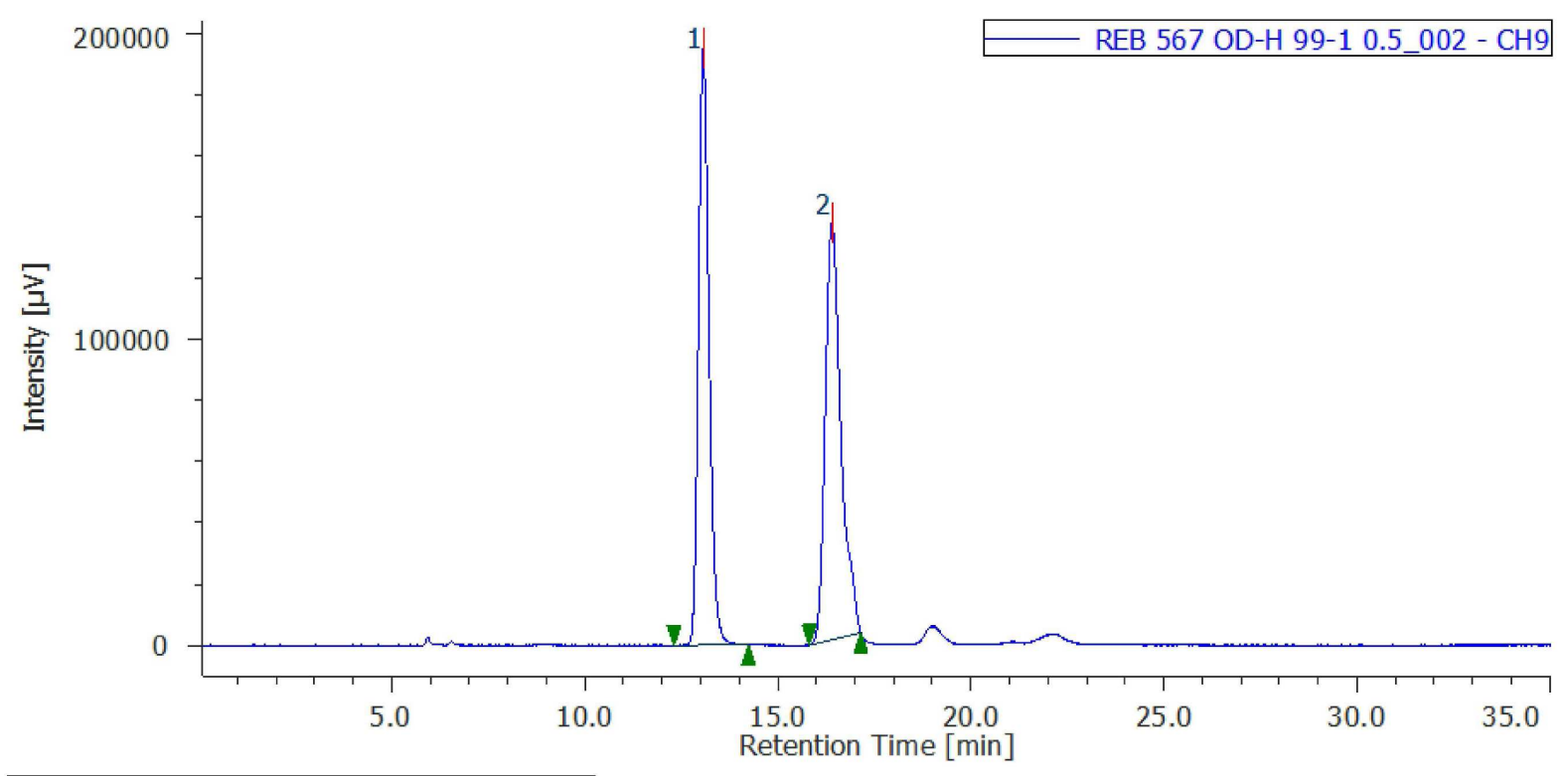

\begin{tabular}{|l|l|l|l|l|l|}
$\#$ Peak Name & CH & tR [min] & Area\% & Peak Start & Peak End \\
\hline
\end{tabular}

\begin{tabular}{|l|l|l|l|l|l|l|}
\hline 1 & Unknown & 9 & 13.064 & 49.218 & 12.304 & 14.244 \\
\hline
\end{tabular}

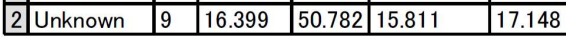

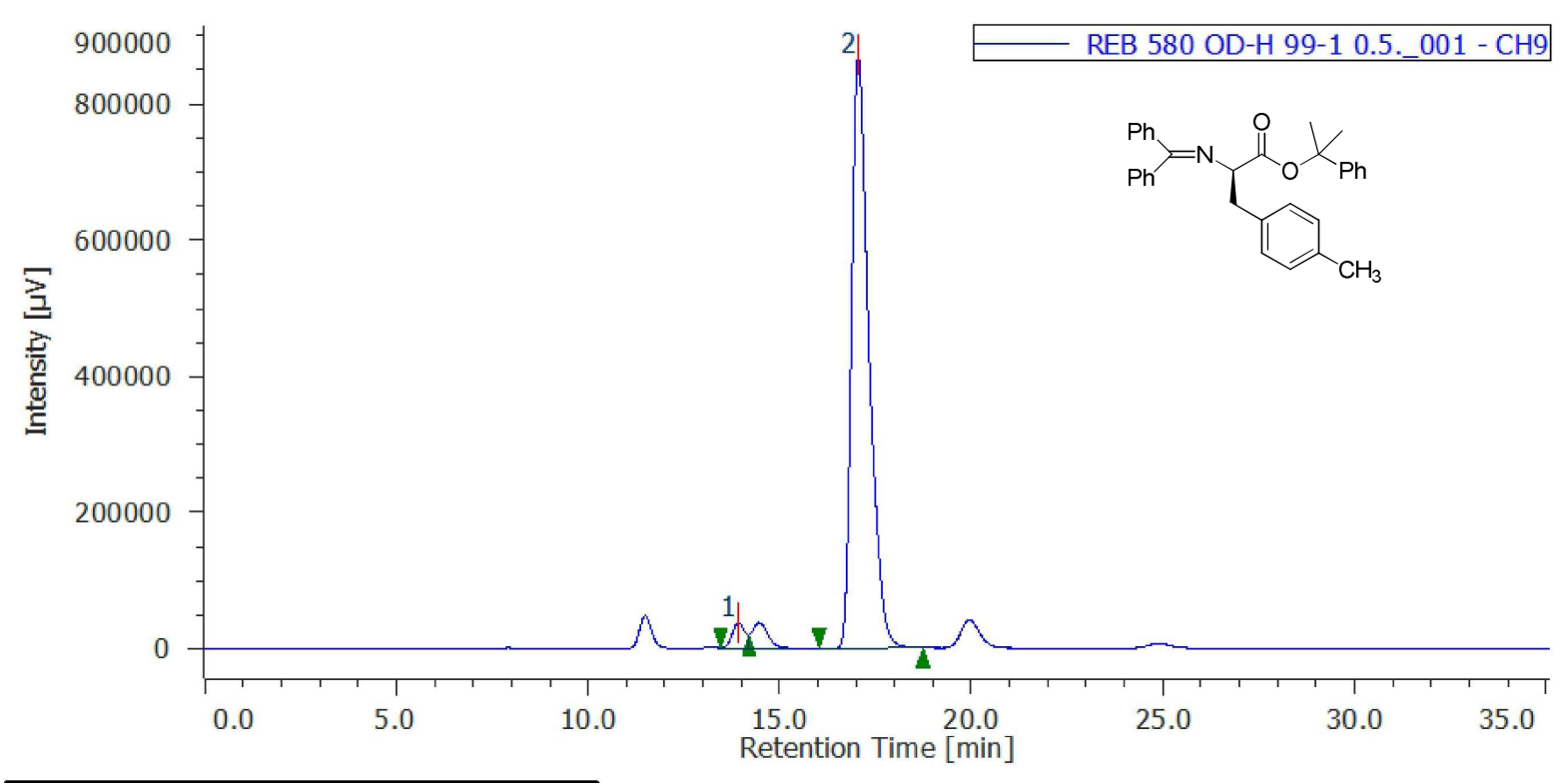

\begin{tabular}{|l|l|l|l|l|l|}
$\#$ Peak Name & $\mathrm{CH}$ & tR $[\mathrm{min}]$ & Area\% & Peak Start & Peak End \\
\hline
\end{tabular}

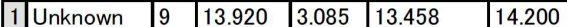

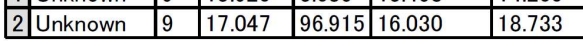


HPLC chromatograms of compound rac-2de and compound $\mathbf{2 d c}$

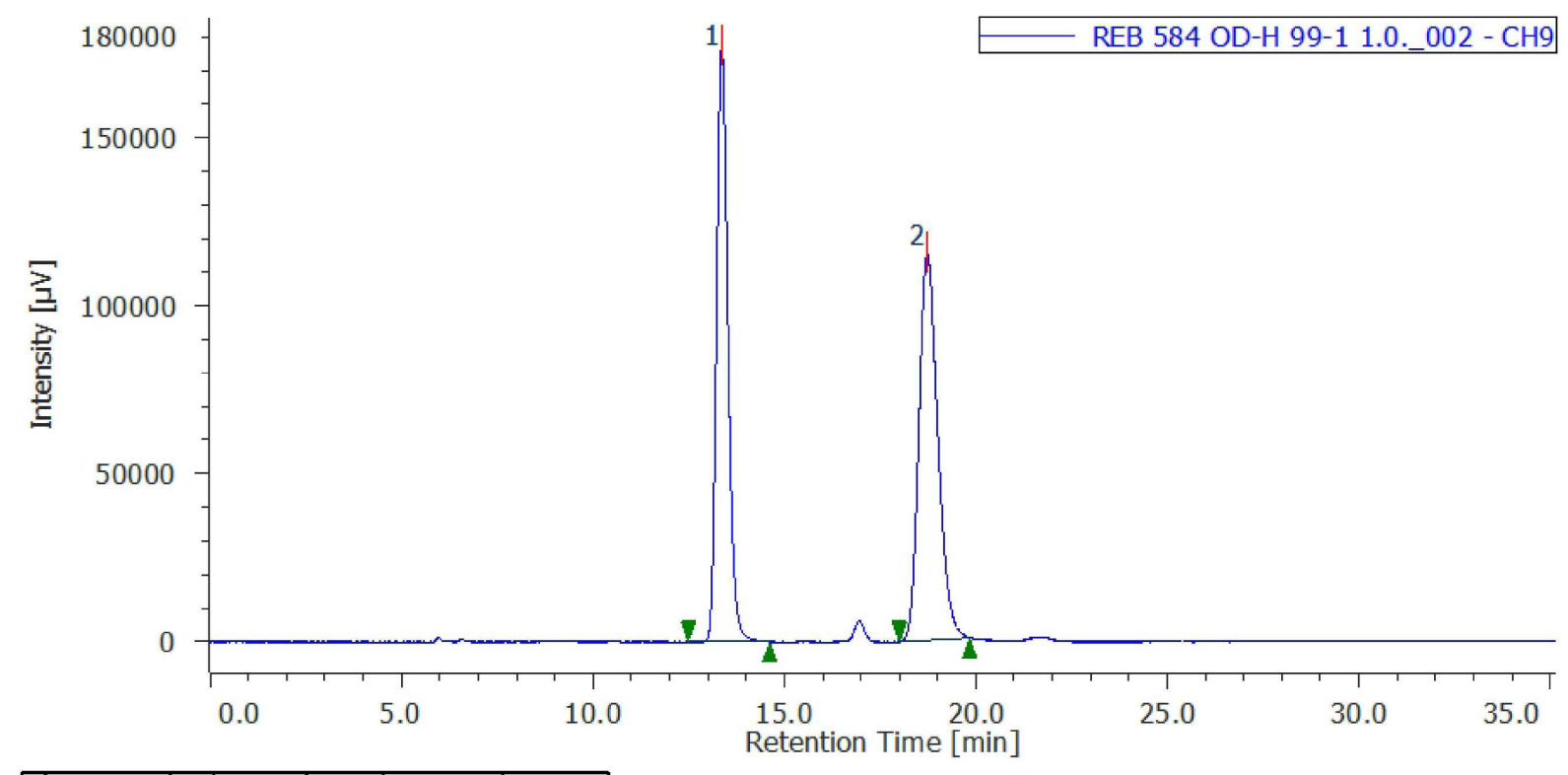

\begin{tabular}{|l|l|l|l|l|l|}
$\#$ Peak Name & $\mathrm{CH}$ & tR [min] & Area\% & Peak Start & Peak End \\
\hline
\end{tabular}

\begin{tabular}{|l|l|l|l|l|l|l|}
\hline 1 & Unknown & 9 & 13.355 & 47.529 & 12.485 & 14.618 \\
\hline
\end{tabular}

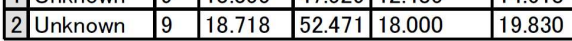

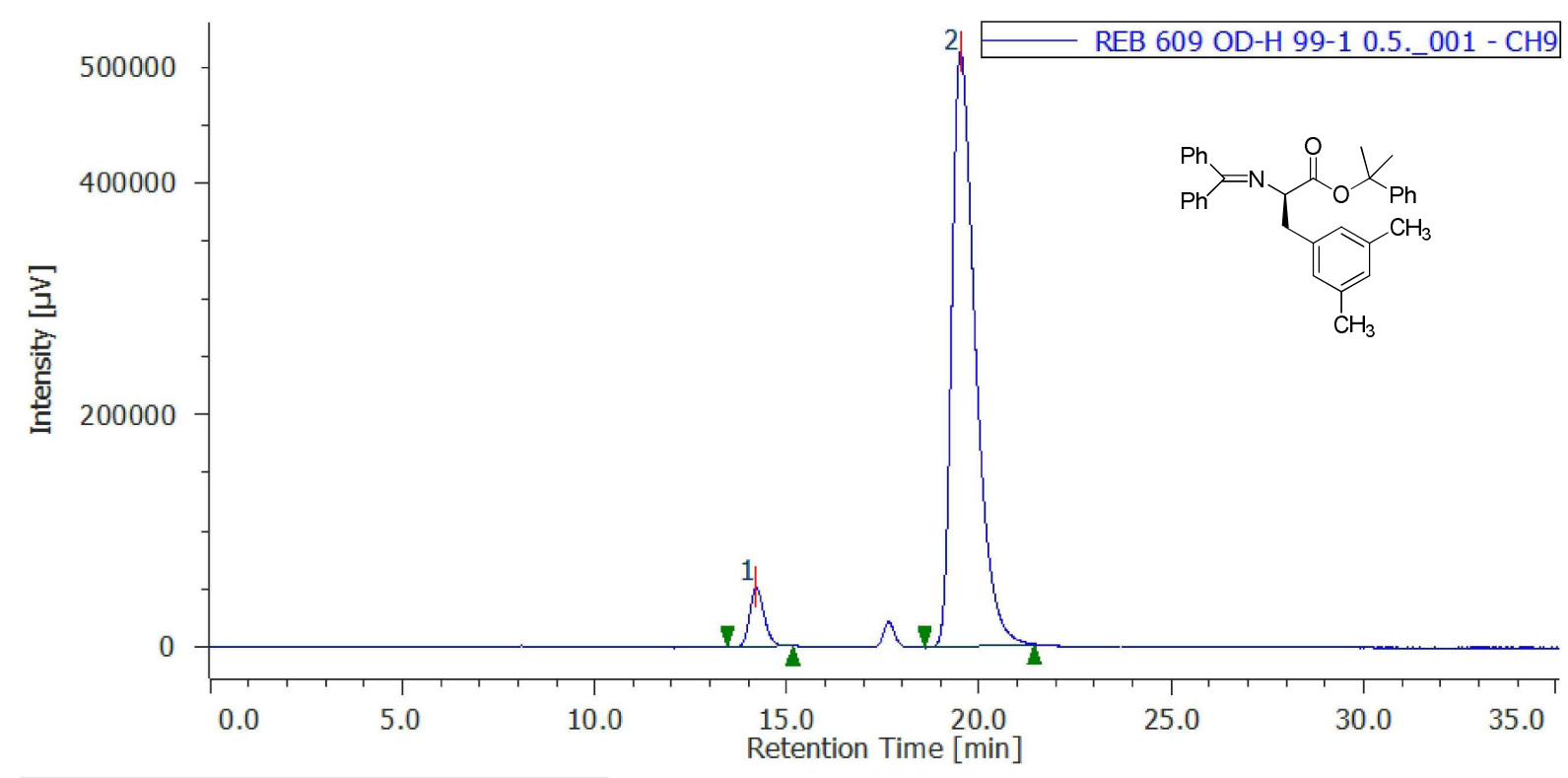

\begin{tabular}{|l|l|l|l|l|l|}
\hline Peak Name & CH & tR [min] & Area\% & Peak Start & Peak End \\
\hline & Unkno & & & \\
\hline
\end{tabular}

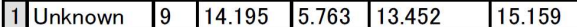

\begin{tabular}{|l|l|l|l|l|l|l|}
\hline 2 & Unknown & 9 & 19.515 & 94.237 & 18.572 & 21.433 \\
\hline
\end{tabular} 
HPLC chromatograms of compound rac-2dd and compound $\mathbf{2 d d}$

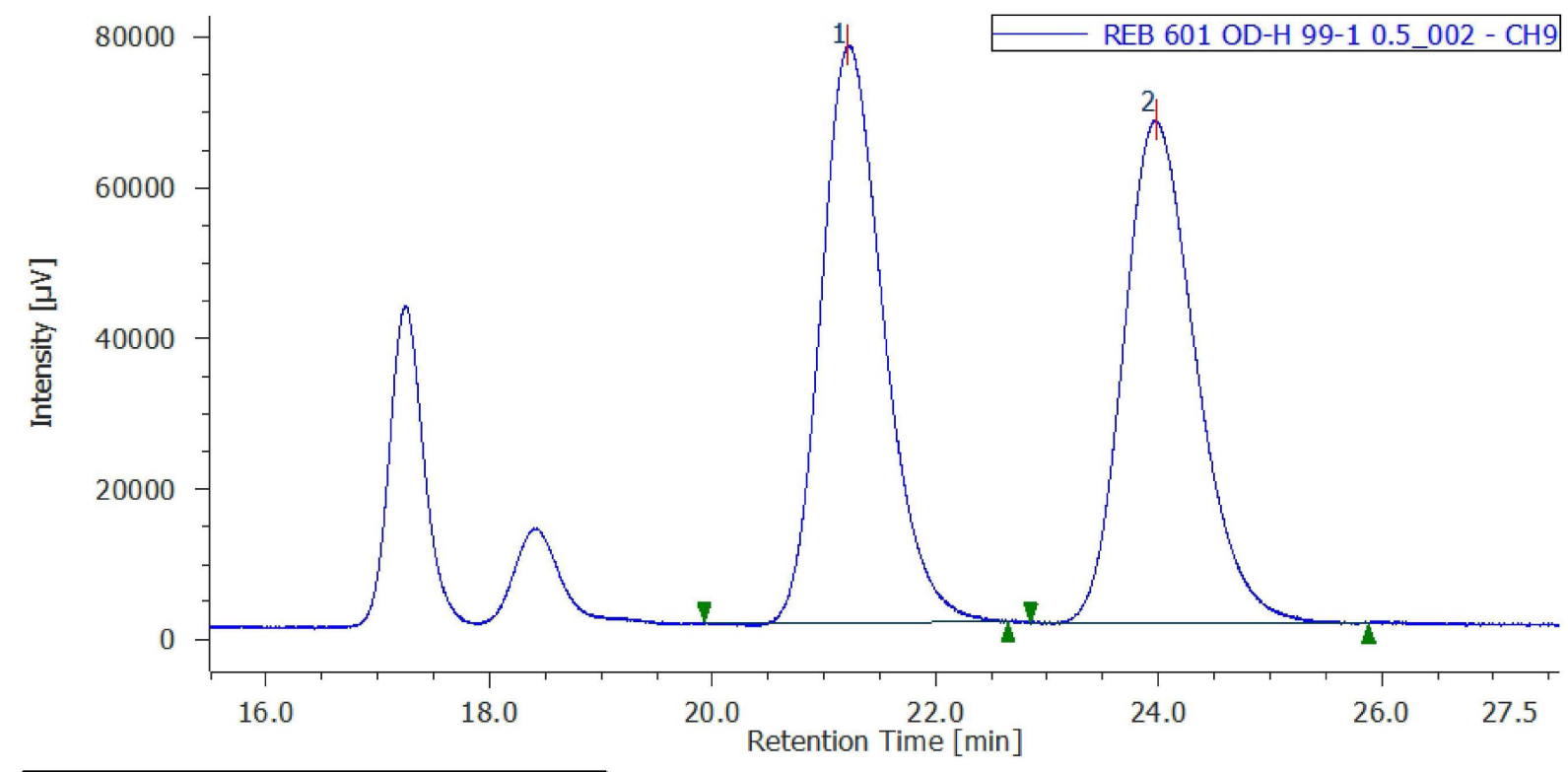

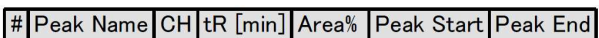

\begin{tabular}{|l|l|l|l|l|l|l|}
\hline 1 & Unknown & 9 & 21.215 & 49.796 & 19.923 & 22.645 \\
\hline
\end{tabular}

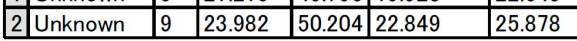

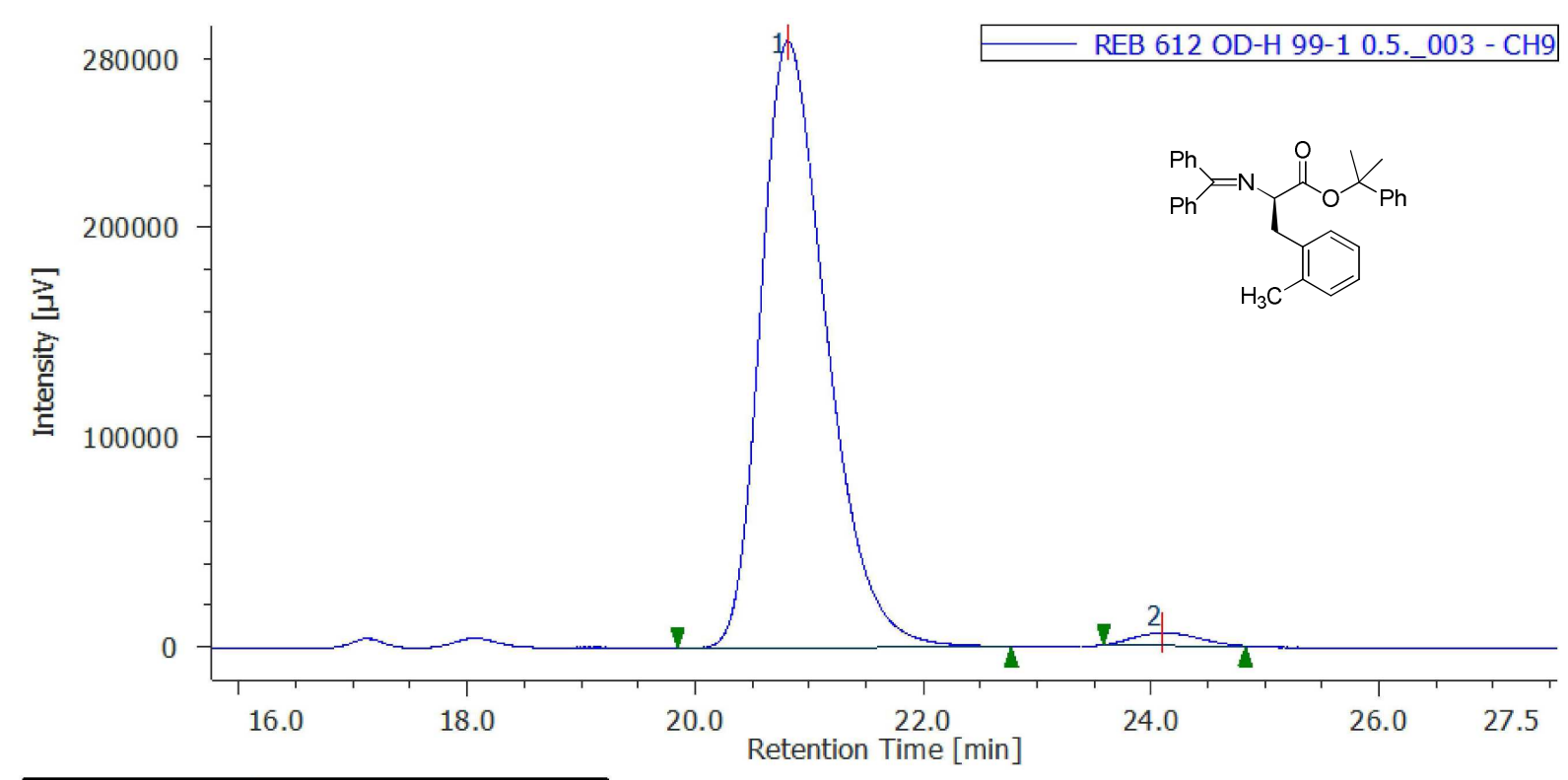

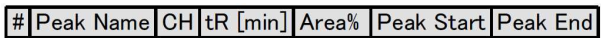

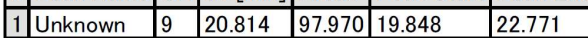

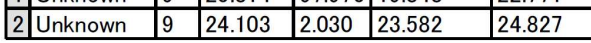


HPLC chromatograms of compound rac-2de and compound 2de
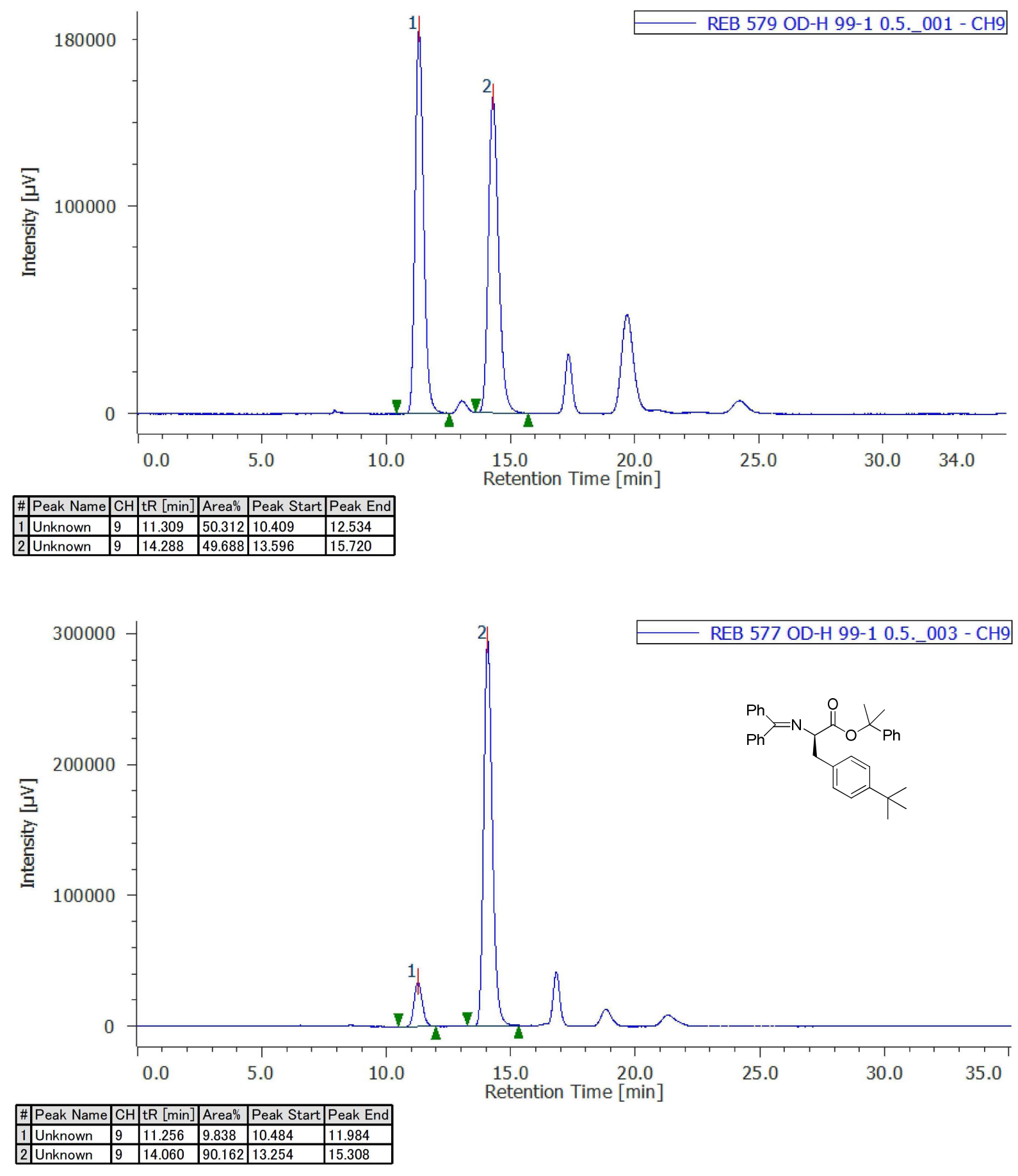
HPLC chromatograms of compound rac-2df and compound $\mathbf{2 d f}$

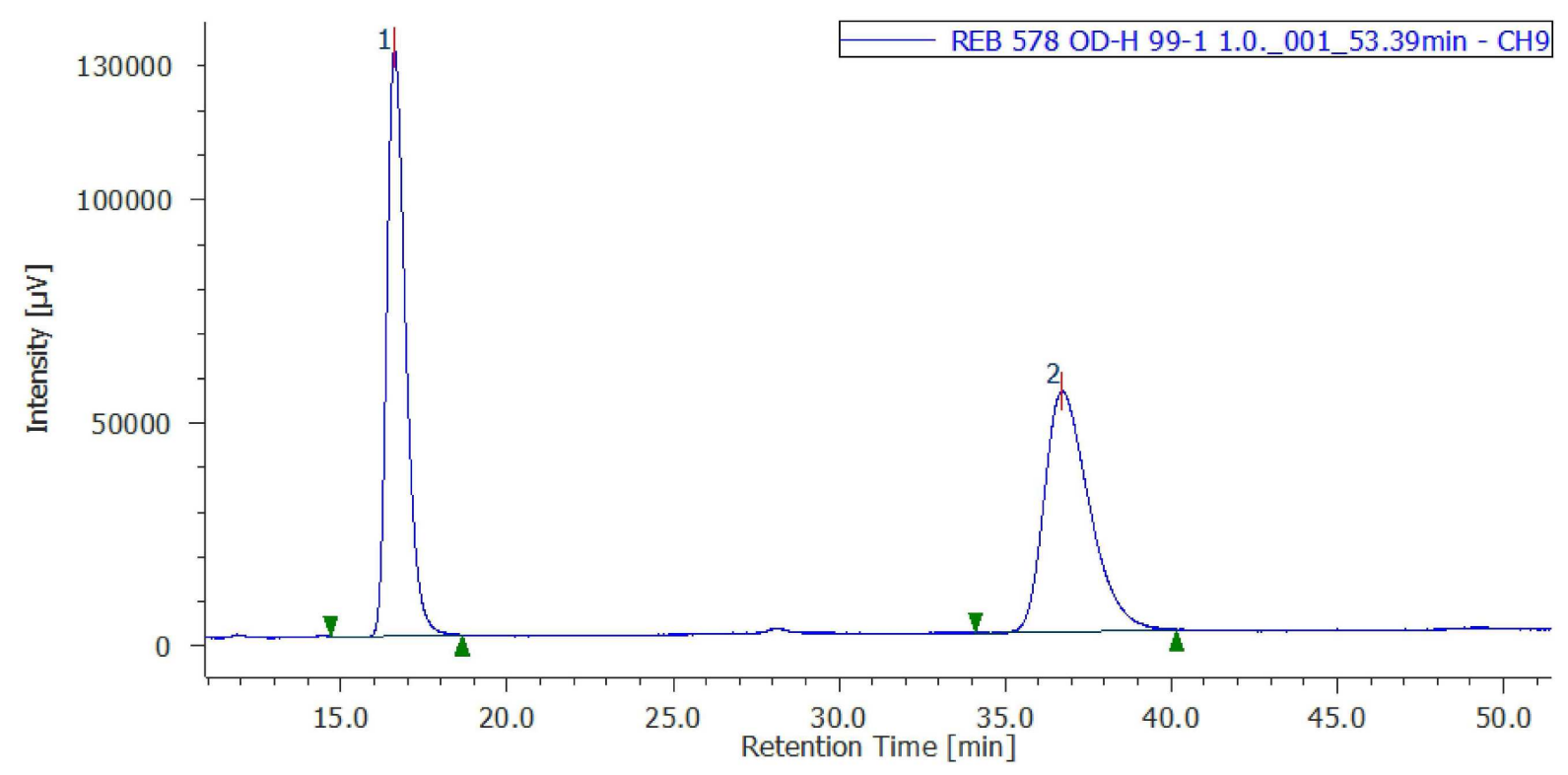

\begin{tabular}{|l|l|l|l|l|l|}
$\#$ Peak Name & CH & tR [min] & Area\% & Peak Start & Peak End \\
\hline
\end{tabular}

\begin{tabular}{|l|l|l|l|l|l|l|}
\hline 1 & Unknown & 9 & 16.622 & 50.496 & 14.699 & 18.647 \\
\hline
\end{tabular}

\begin{tabular}{|l|l|l|l|l|l|l|}
\hline 2 & Unknown & 9 & 36.718 & 49.504 & 34.102 & 40.149 \\
\hline
\end{tabular}

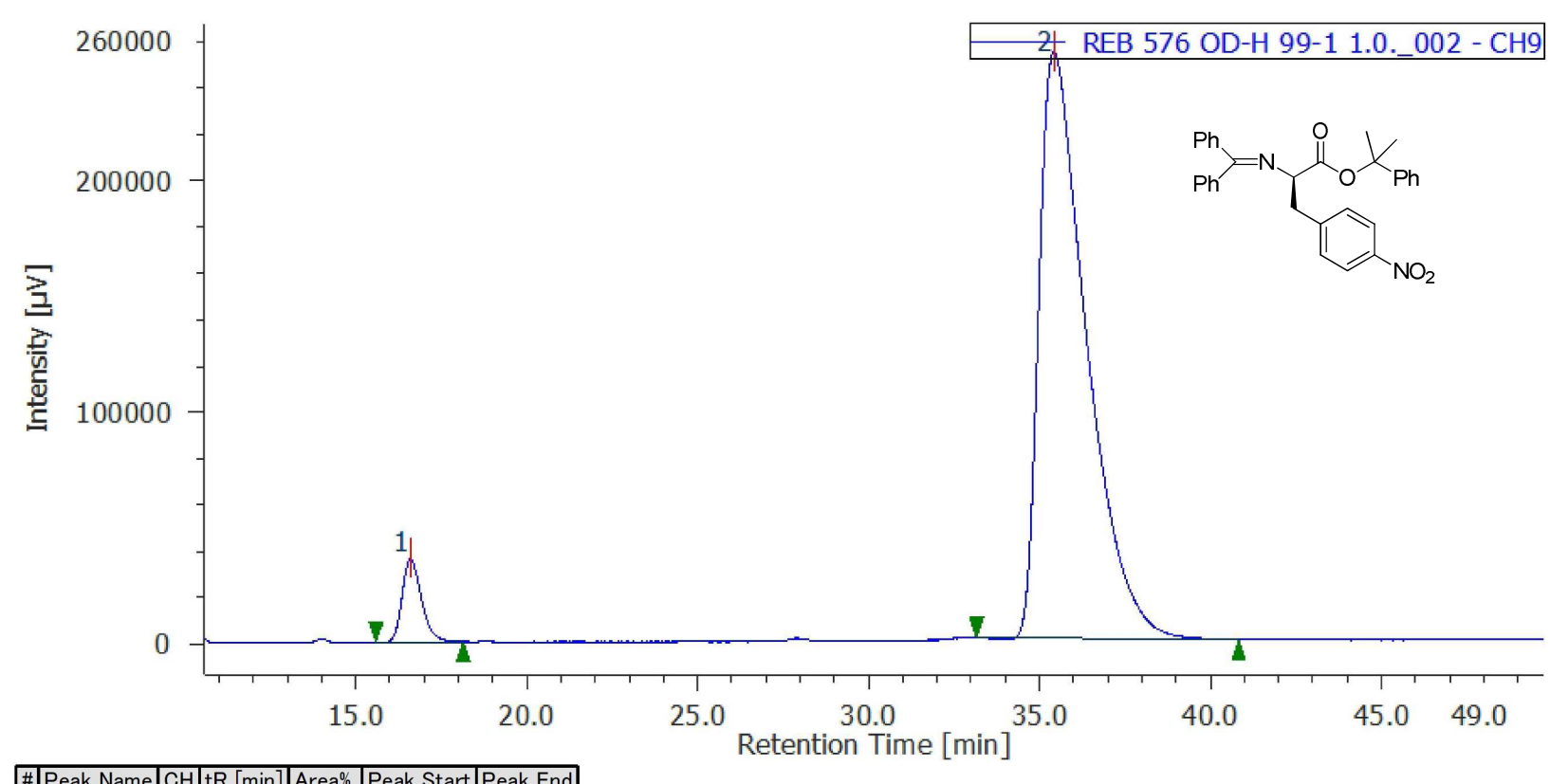

\begin{tabular}{|l|l|l|l|l|l|}
\hline Peak Name & $\mathrm{CH}$ & tR [min] & Area\% & Peak Start & Peak End \\
\hline
\end{tabular}

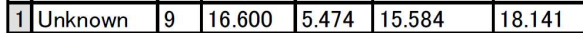

\begin{tabular}{|l|l|l|l|l|l|l|}
\hline 2 & Unknown & 9 & 35.421 & 94.526 & 33.157 & 40.827 \\
\hline
\end{tabular} 
HPLC chromatograms of compound rac-2dg and compound $\mathbf{2 d g}$

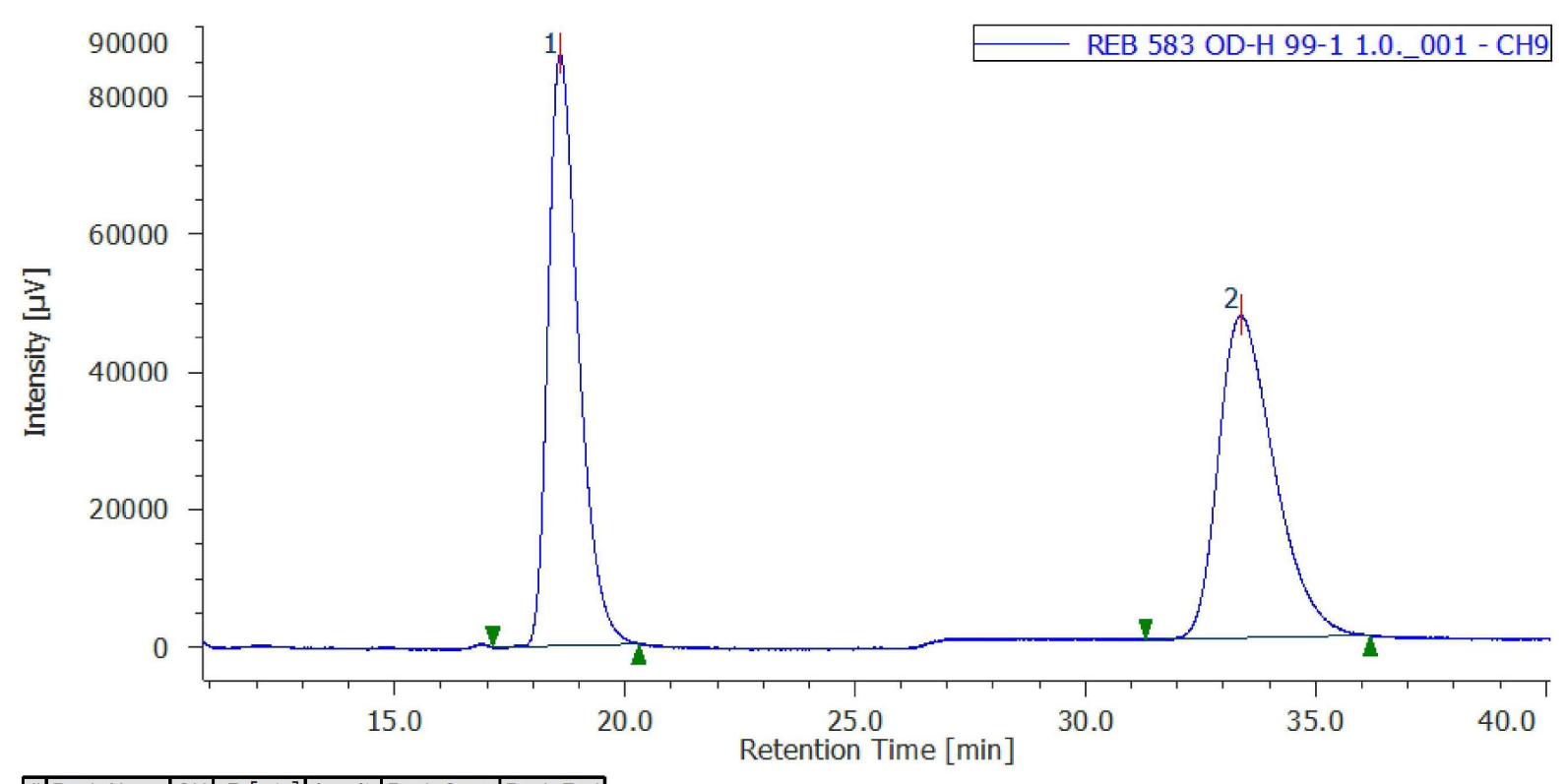

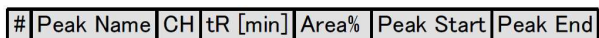

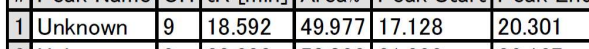

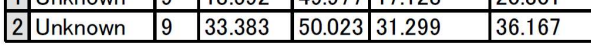

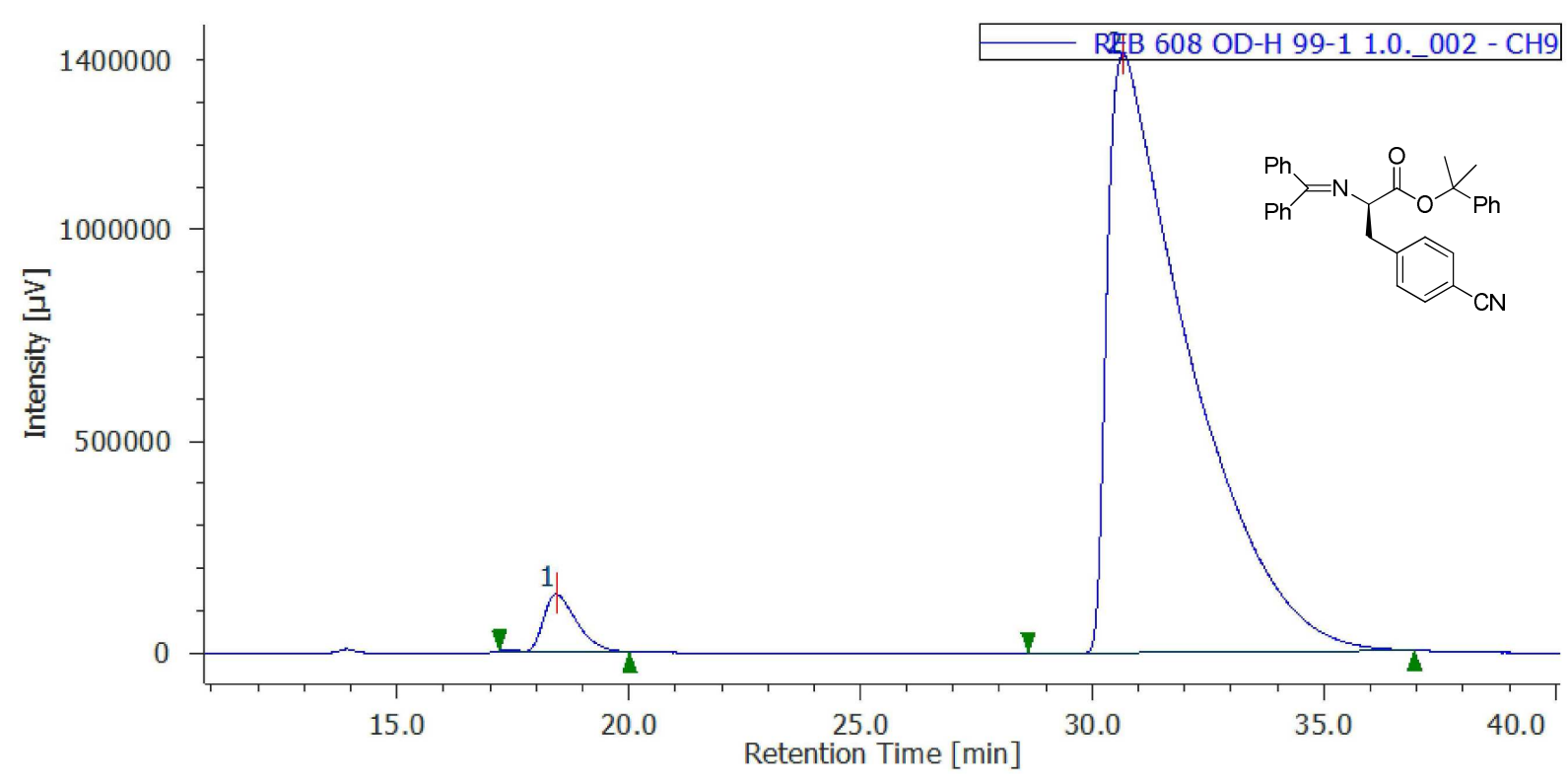

\begin{tabular}{|l|l|l|l|l|l|}
\hline \#eak Name & CH & tR [min] & Area\% & Peak Start & Peak End \\
\hline
\end{tabular}

\begin{tabular}{|l|l|l|l|l|l|l|}
\hline 1 & Unknown & 9 & 18.435 & 3.685 & 17.204 & 20.017 \\
\hline
\end{tabular}

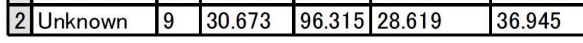


HPLC chromatograms of compound rac-2 dh and compound $\mathbf{2 d h}$

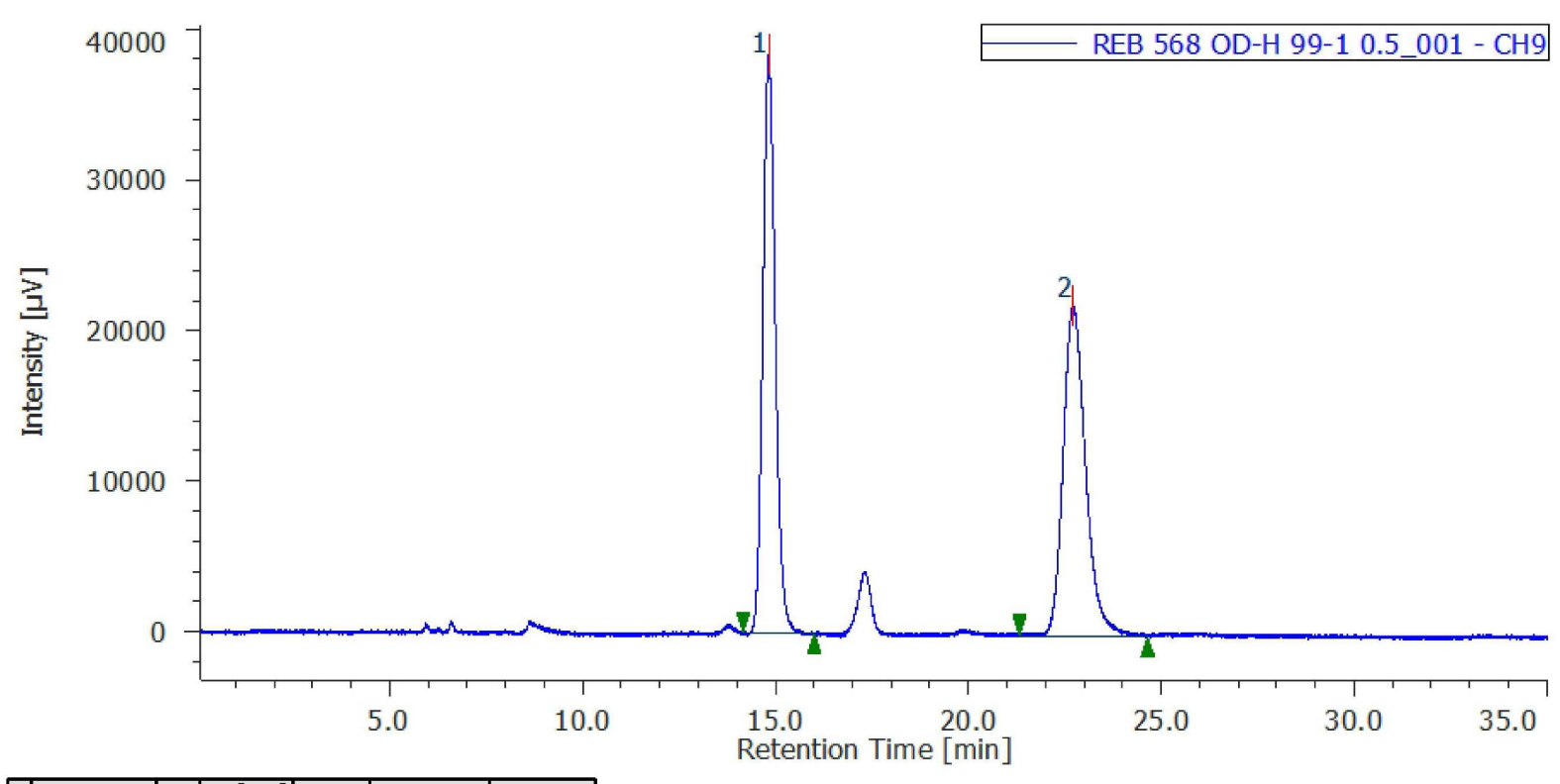

\begin{tabular}{|l|l|l|l|l|l|}
\hline Peak Name & CH & tR [min] & Area\% & Peak Start & Peak End \\
\hline
\end{tabular}

\begin{tabular}{|l|l|l|l|l|l|l|}
\hline 1 & Unknown & 9 & 14.817 & 49.517 & 14.162 & 16.003 \\
\hline
\end{tabular}

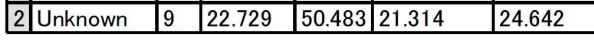

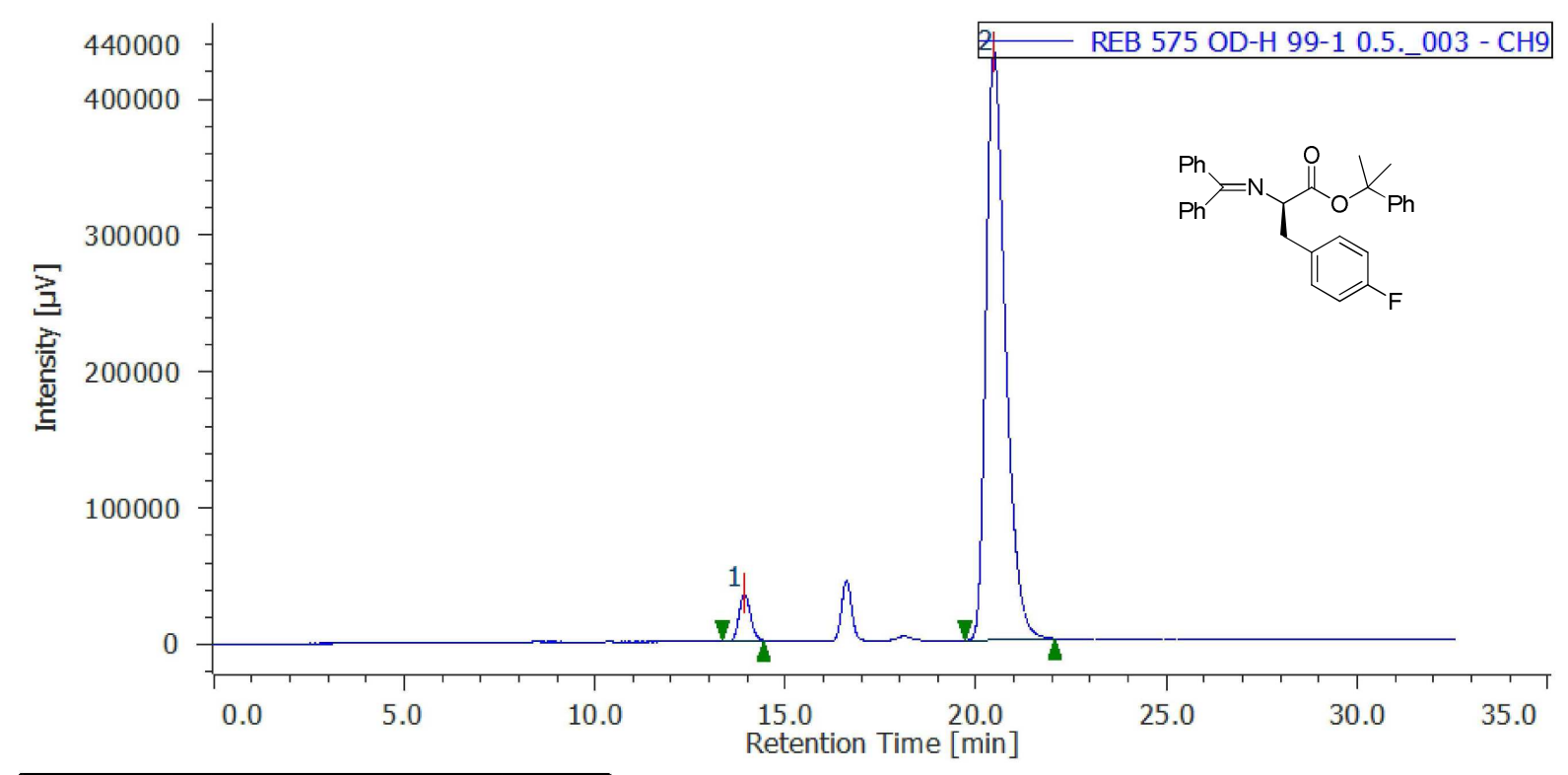

\begin{tabular}{|l|l|l|l|l|l|}
$\#$ Peak Name & $\mathrm{CH}$ & tR $[\mathrm{min}]$ & Area\% & Peak Start & Peak End \\
\hline
\end{tabular}

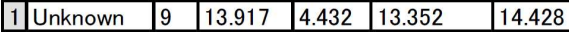

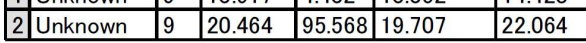


HPLC chromatograms of compound rac-2di and compound 2di

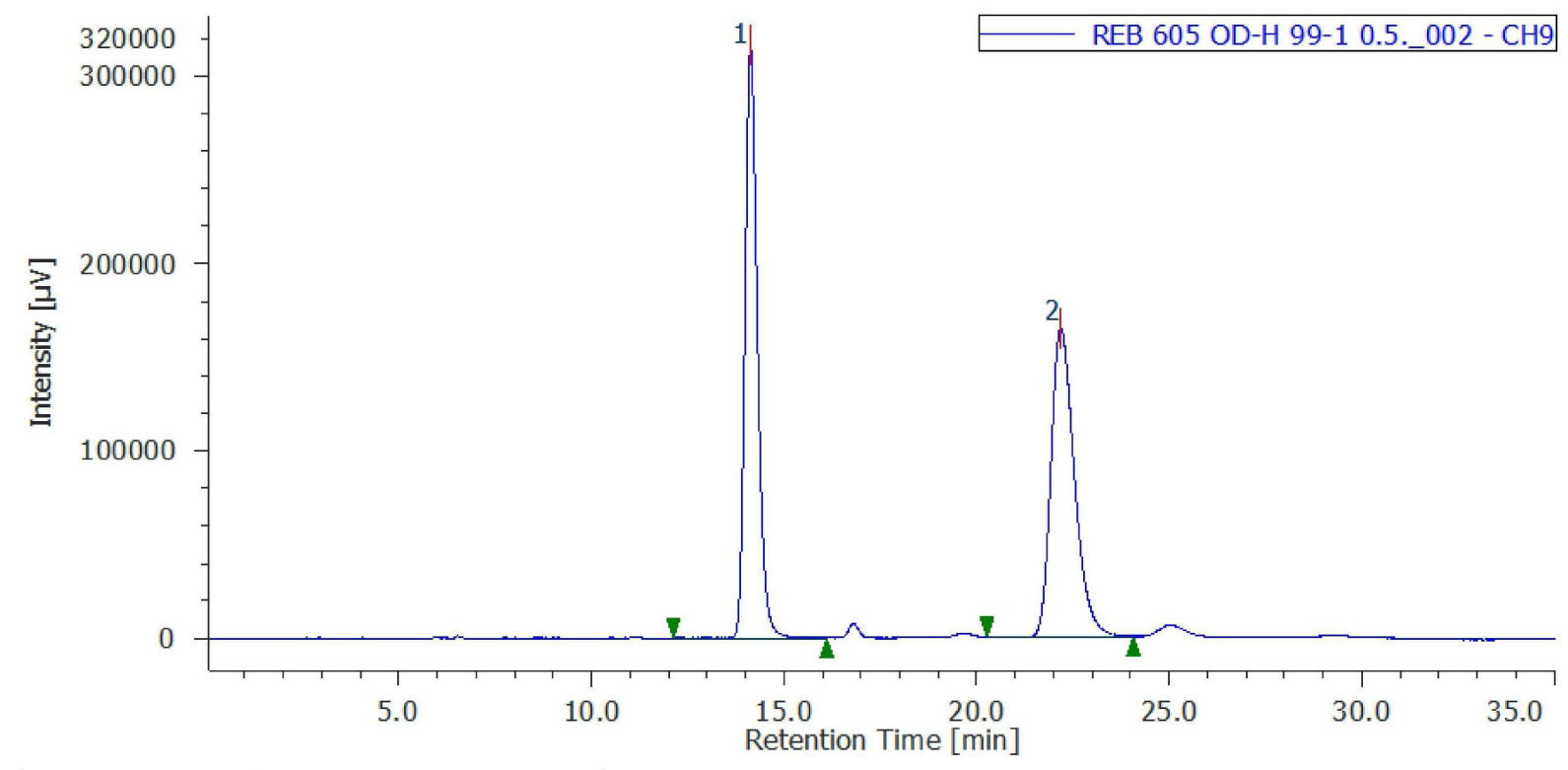

\begin{tabular}{|l|l|l|l|l|l|}
$\#$ Peak Name & CH & tR [min] & Area\% & Peak Start & Peak End \\
\hline
\end{tabular}

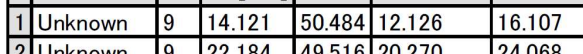

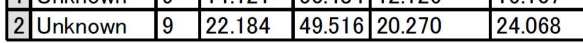

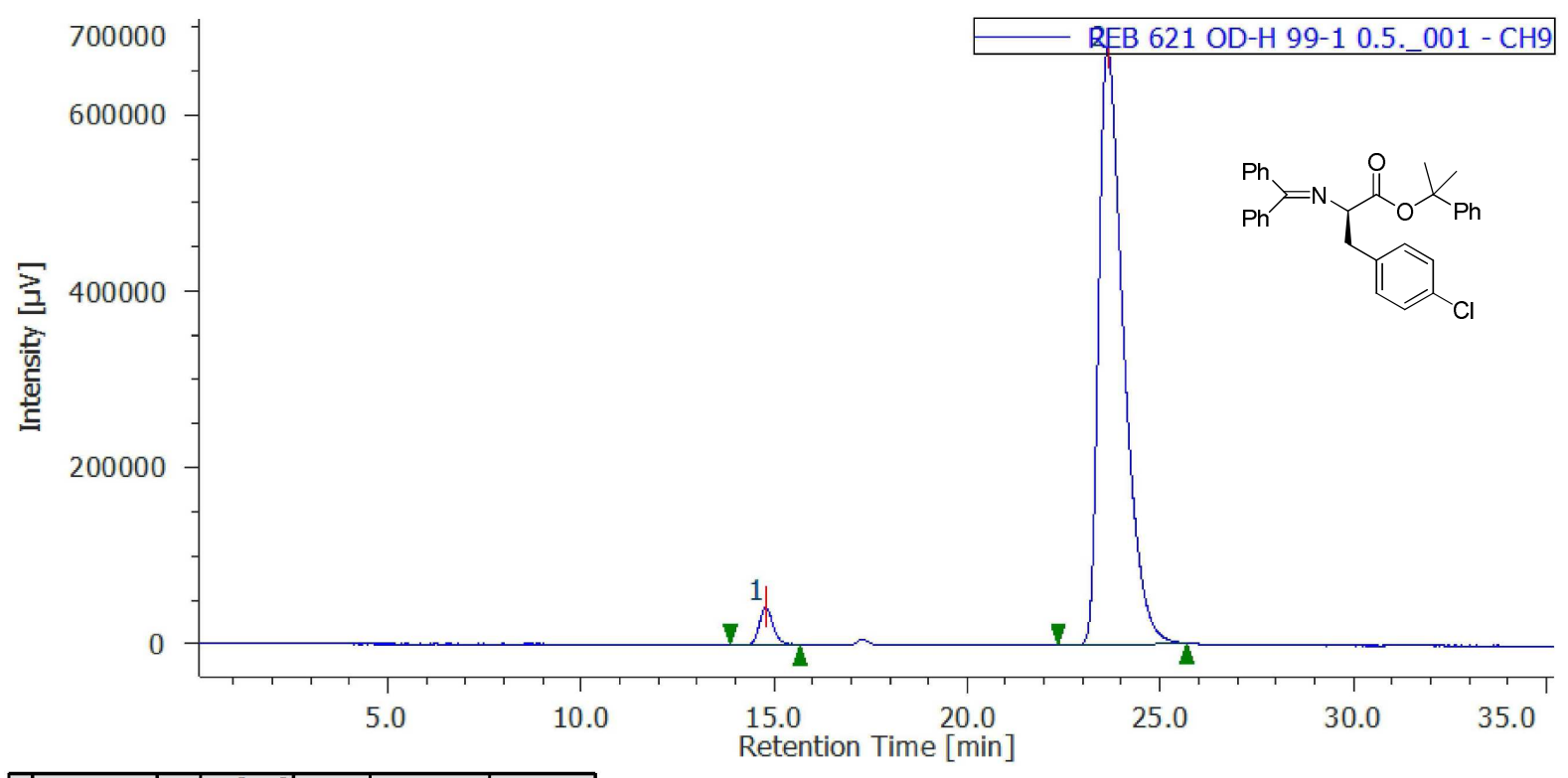

\begin{tabular}{|l|l|l|l|l|l|}
$\#$ Peak Name & CH & tR [min] & Area\% & Peak Start & Peak End \\
\hline
\end{tabular}

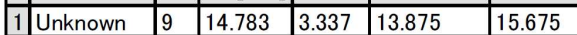

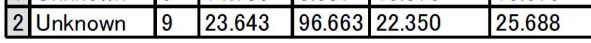


HPLC chromatograms of compound $\mathbf{r a c}-\mathbf{2} \mathbf{d j}$ and compound $\mathbf{2 d j}$

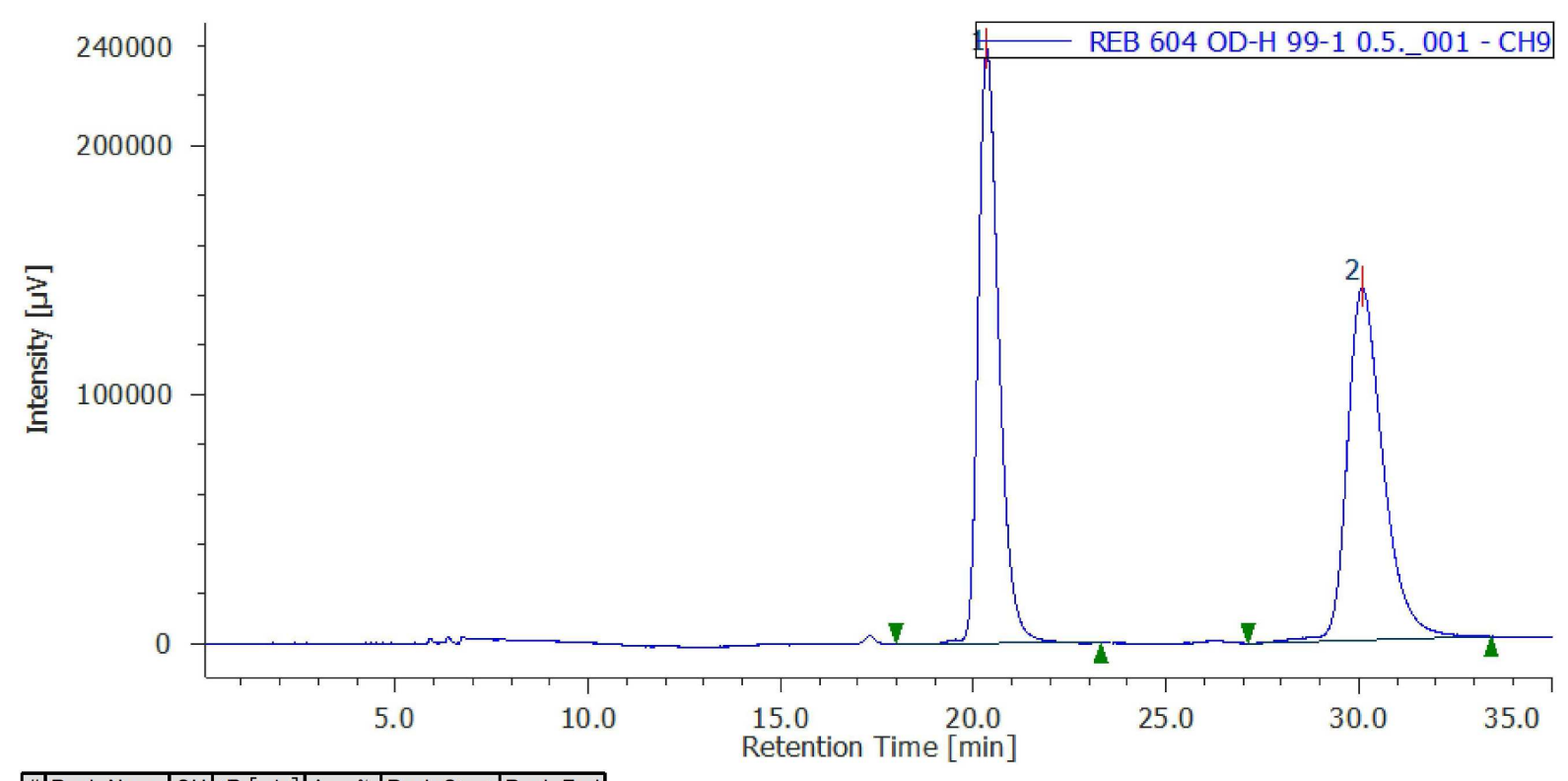

\begin{tabular}{|l|l|l|l|l|l|}
$\#$ Peak Name & $\mathrm{CH}$ & tR [min] & Area\% & Peak Start & Peak End \\
\hline
\end{tabular}

\begin{tabular}{|l|l|l|l|l|l|l|}
\hline 1 & Unknown & 9 & 20.333 & 49.334 & 17.986 & 23.297 \\
\hline
\end{tabular}

\begin{tabular}{|l|l|l|l|l|l|l|}
\hline 2 & Unknown & 9 & 30.067 & 50.666 & 27.120 & 33.422 \\
\hline
\end{tabular}

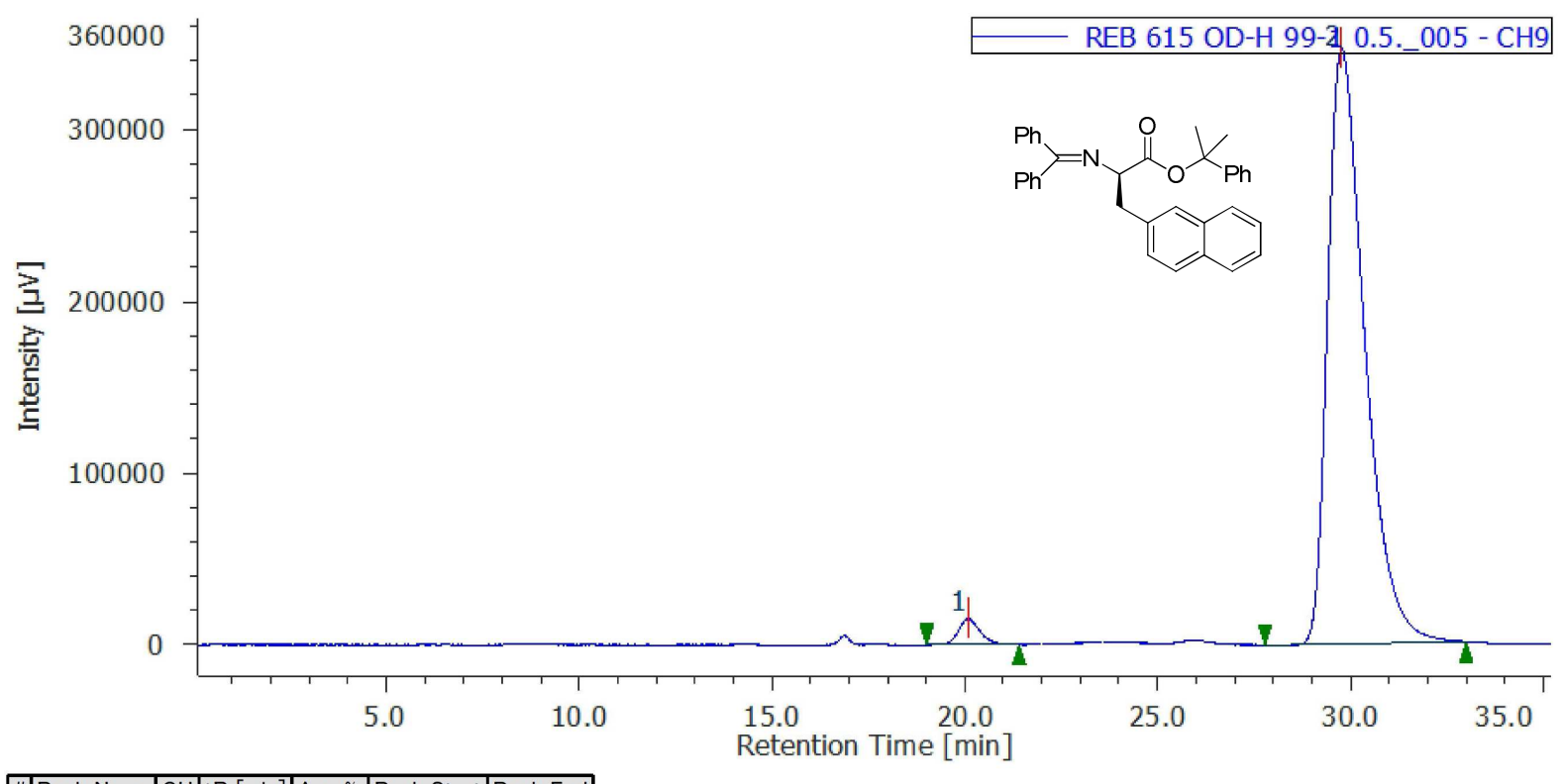

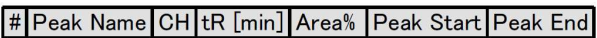

\begin{tabular}{l|l|l|l|l|l|l|}
\hline 1 & Unknown & 9 & 20.087 & 2.476 & 19.006 & 21.395 \\
\hline
\end{tabular}

\begin{tabular}{|l|l|l|l|l|l|l|}
\hline 2 & Unknown & 9 & 29.760 & 97.524 & 27.776 & 32.996 \\
\hline
\end{tabular} 
HPLC chromatograms of compound rac-2 dk and compound $\mathbf{2 d k}$

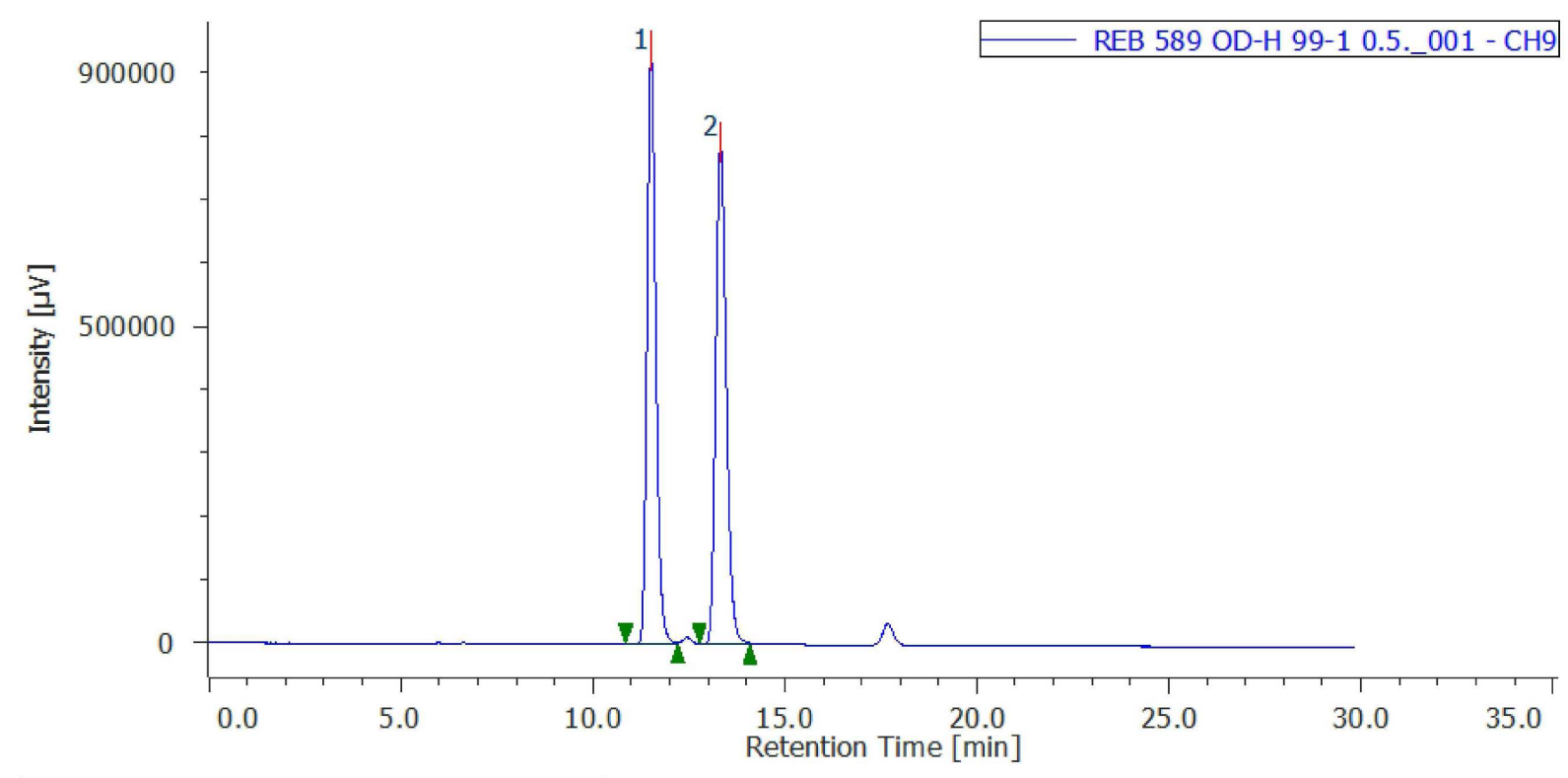

\begin{tabular}{|l|l|l|l|l|l|}
\hline Peak Name & CH & tR [min] & Area\% & Peak Start & Peak End \\
\hline
\end{tabular}

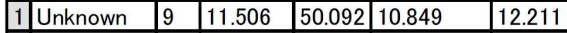

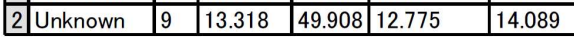

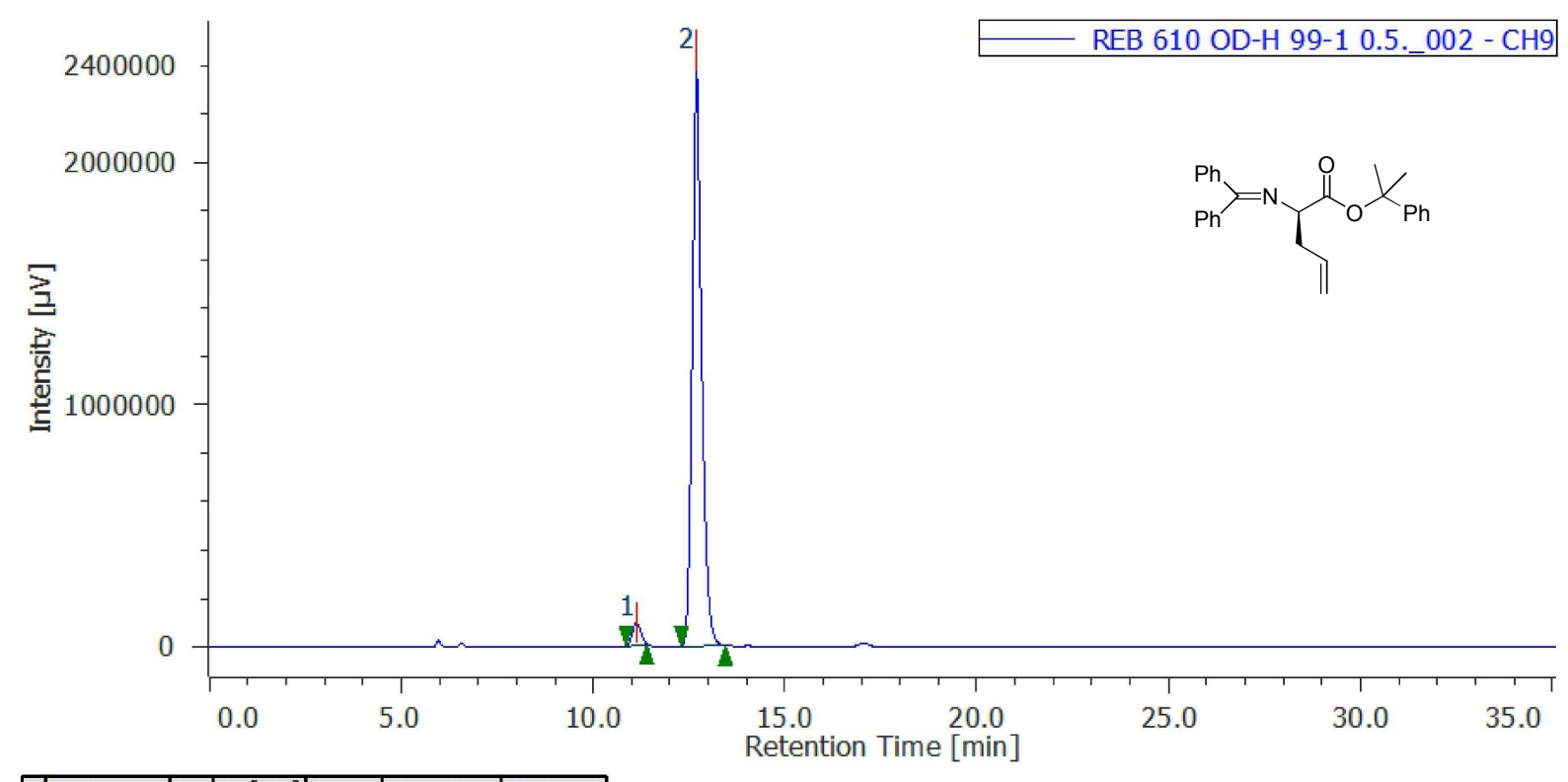

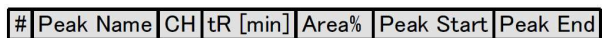

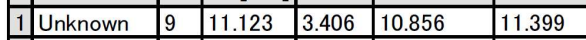

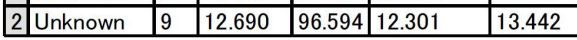


HPLC chromatograms of compound rac-2 dm and compound $\mathbf{2 d m}$

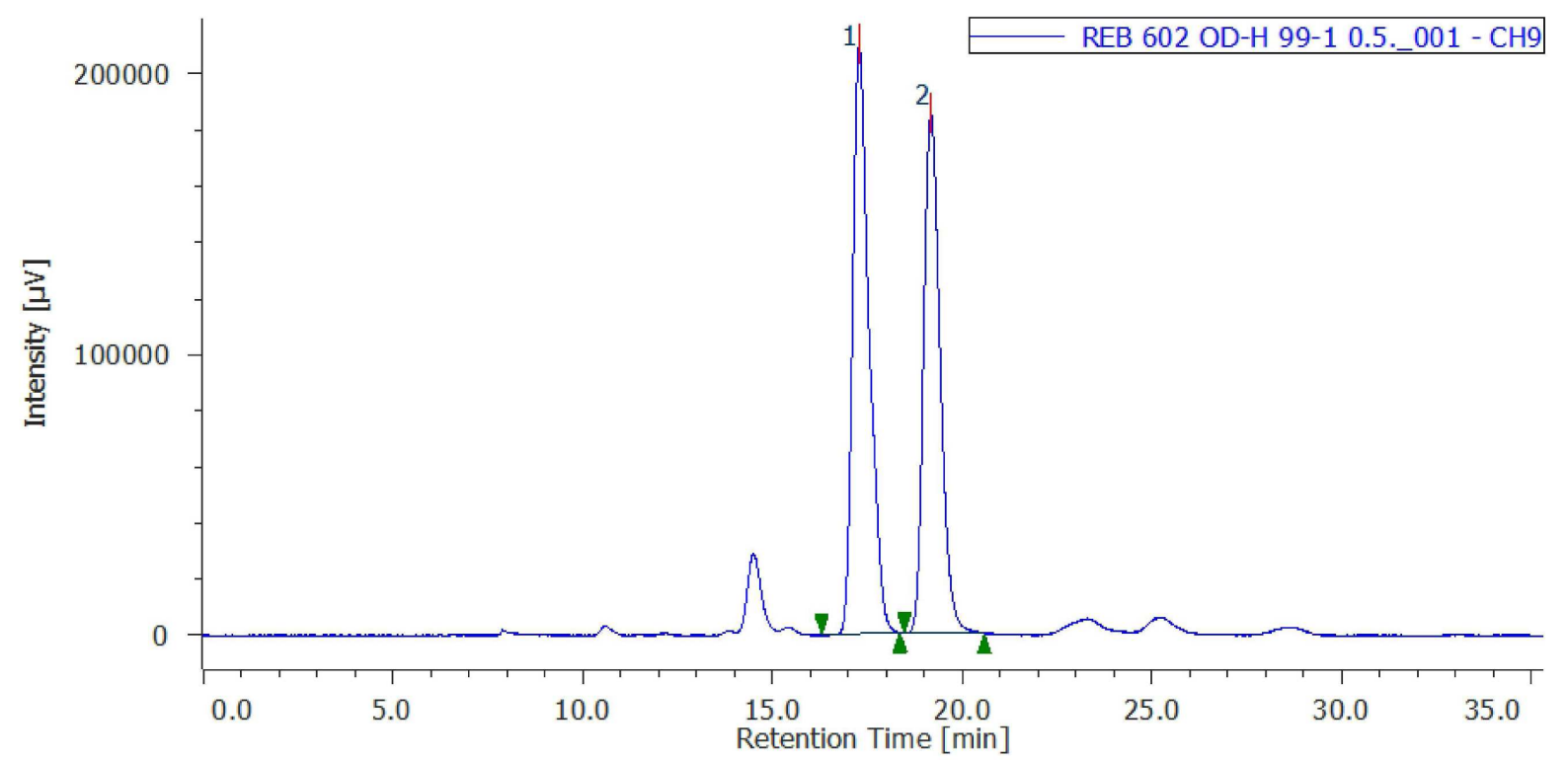

\begin{tabular}{|l|l|l|l|l|l|}
$\#$ Peak Name & CH & tR [min] & Area\% & Peak Start & Peak End \\
\hline
\end{tabular}

\begin{tabular}{|l|l|l|l|l|l|l|}
\hline 1 & Unknown & 9 & 17.287 & 52.705 & 16.293 & 18.351 \\
\hline
\end{tabular}

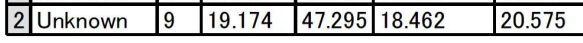

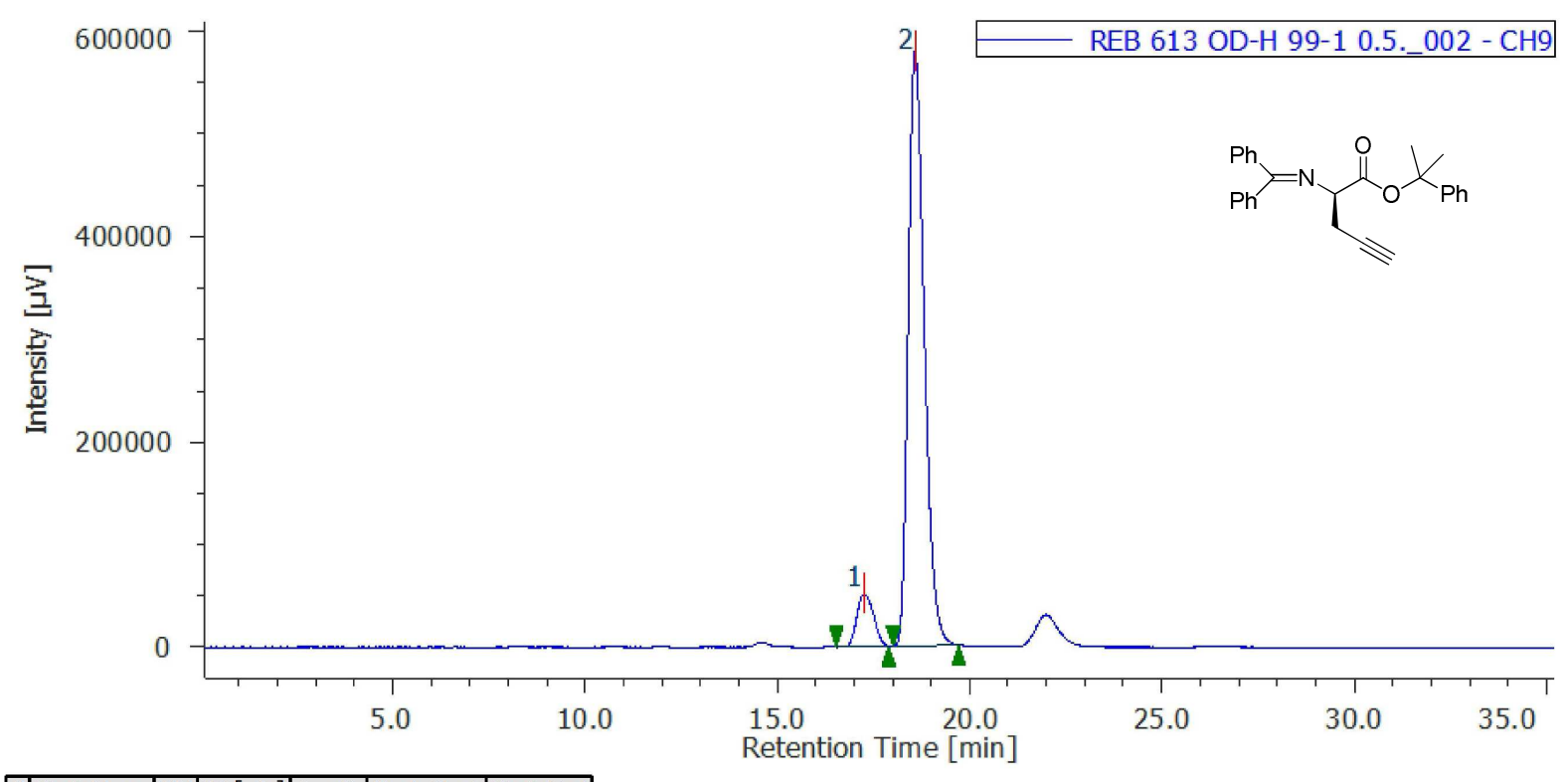

\begin{tabular}{|l|l|l|l|l|l|}
$\#$ Peak Name & $\mathrm{CH}$ & tR [min] & Area\% & Peak Start & Peak End \\
\hline
\end{tabular}

\begin{tabular}{|l|l|l|l|l|l|l|}
\hline 1 & Unknown & 9 & 17.256 & 8.319 & 16.541 & 17.897 \\
\hline
\end{tabular}

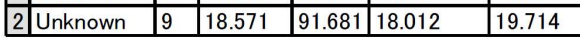


HPLC chromatograms of compound rac-2dn and compound $\mathbf{2 d n}$

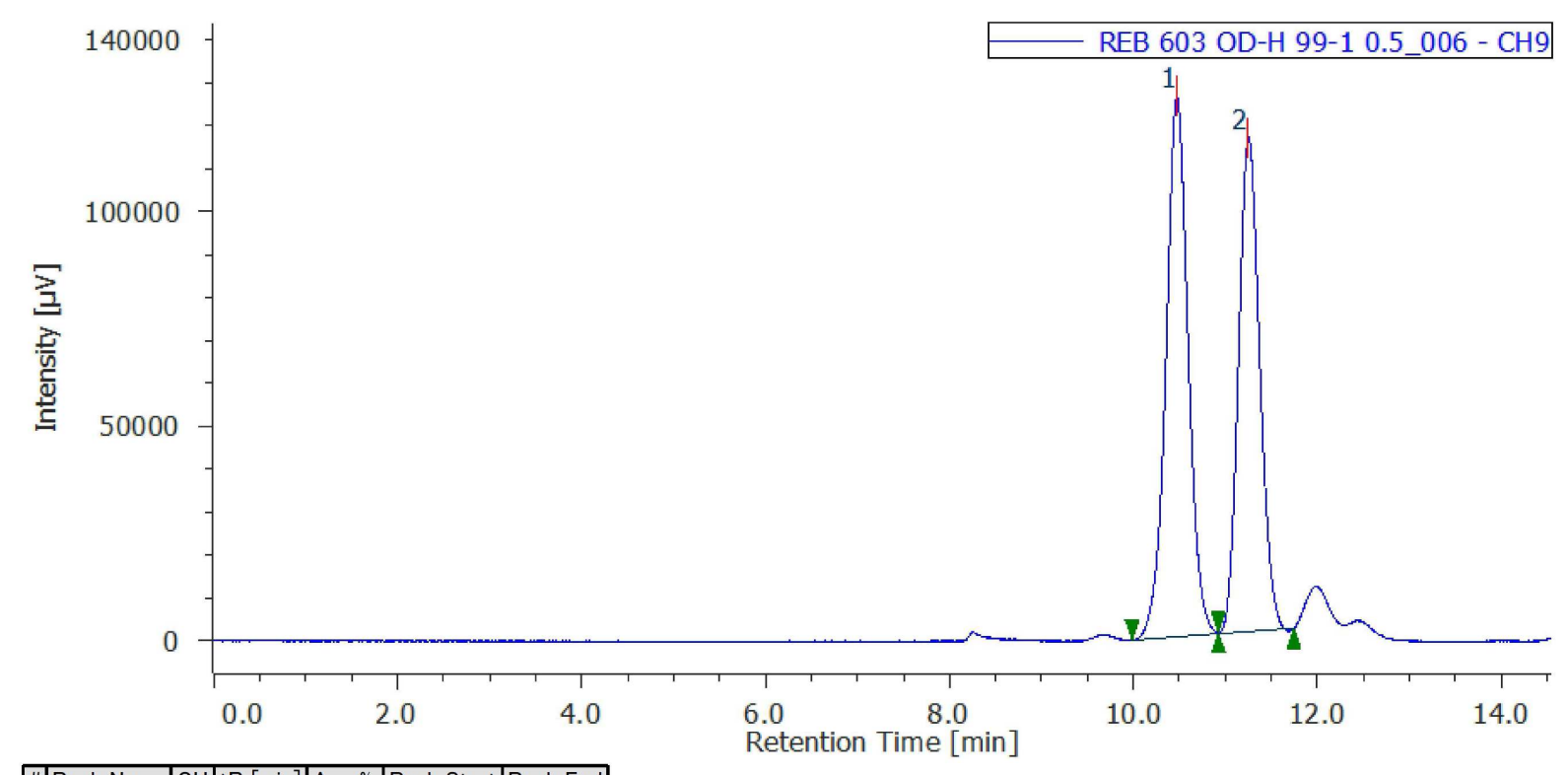

\begin{tabular}{|l|l|l|l|l|l|}
$\#$ Peak Name & $\mathrm{CH}$ & tR [min] & Area\% & Peak Start & Peak End \\
\hline
\end{tabular}

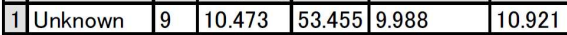

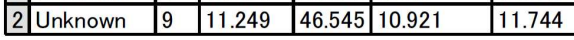

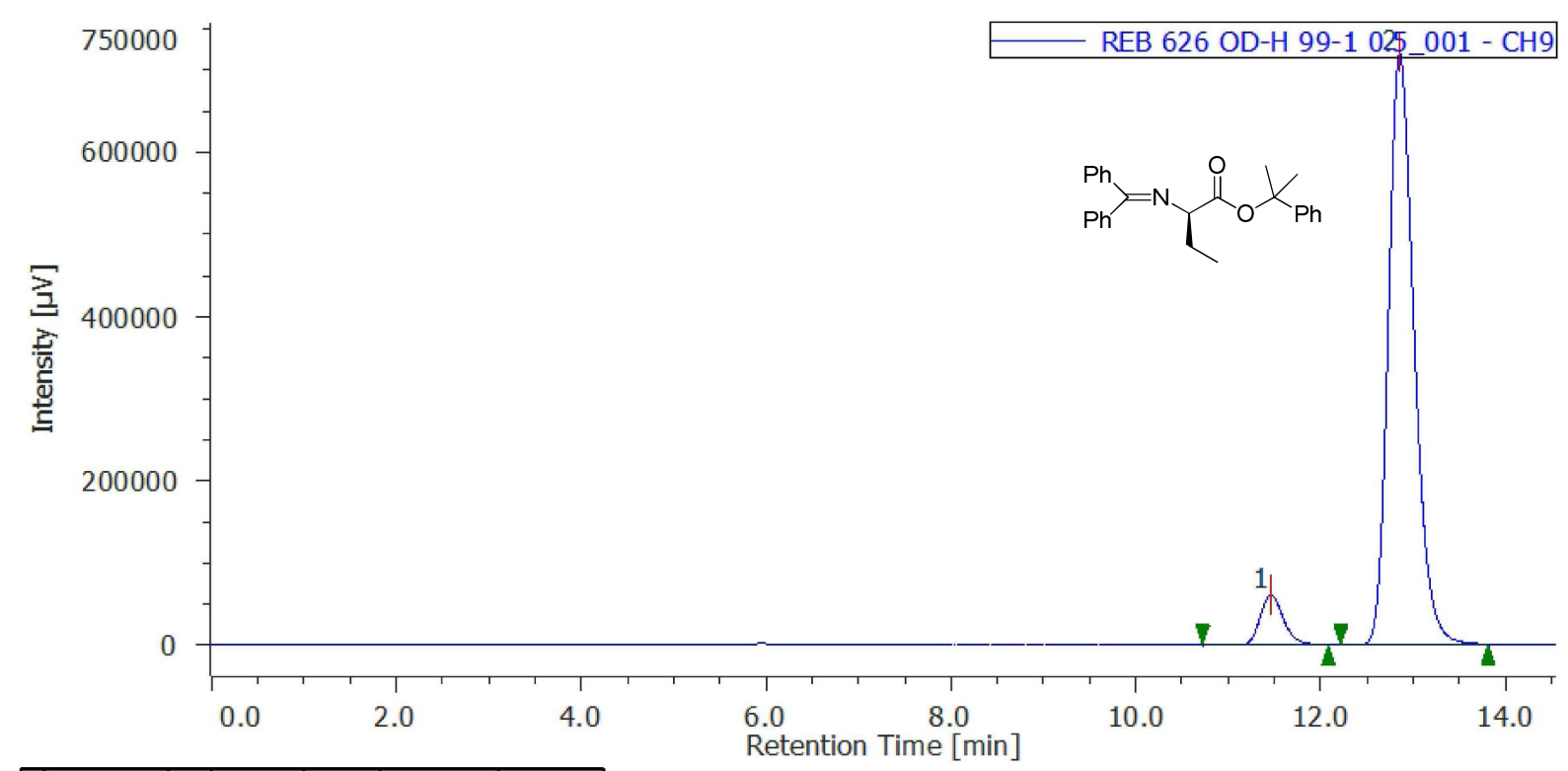

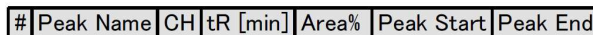

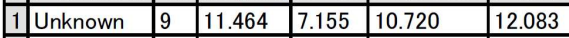

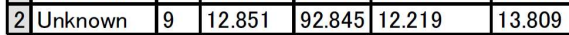


HPLC chromatograms of compound rac-2do and compound 2do

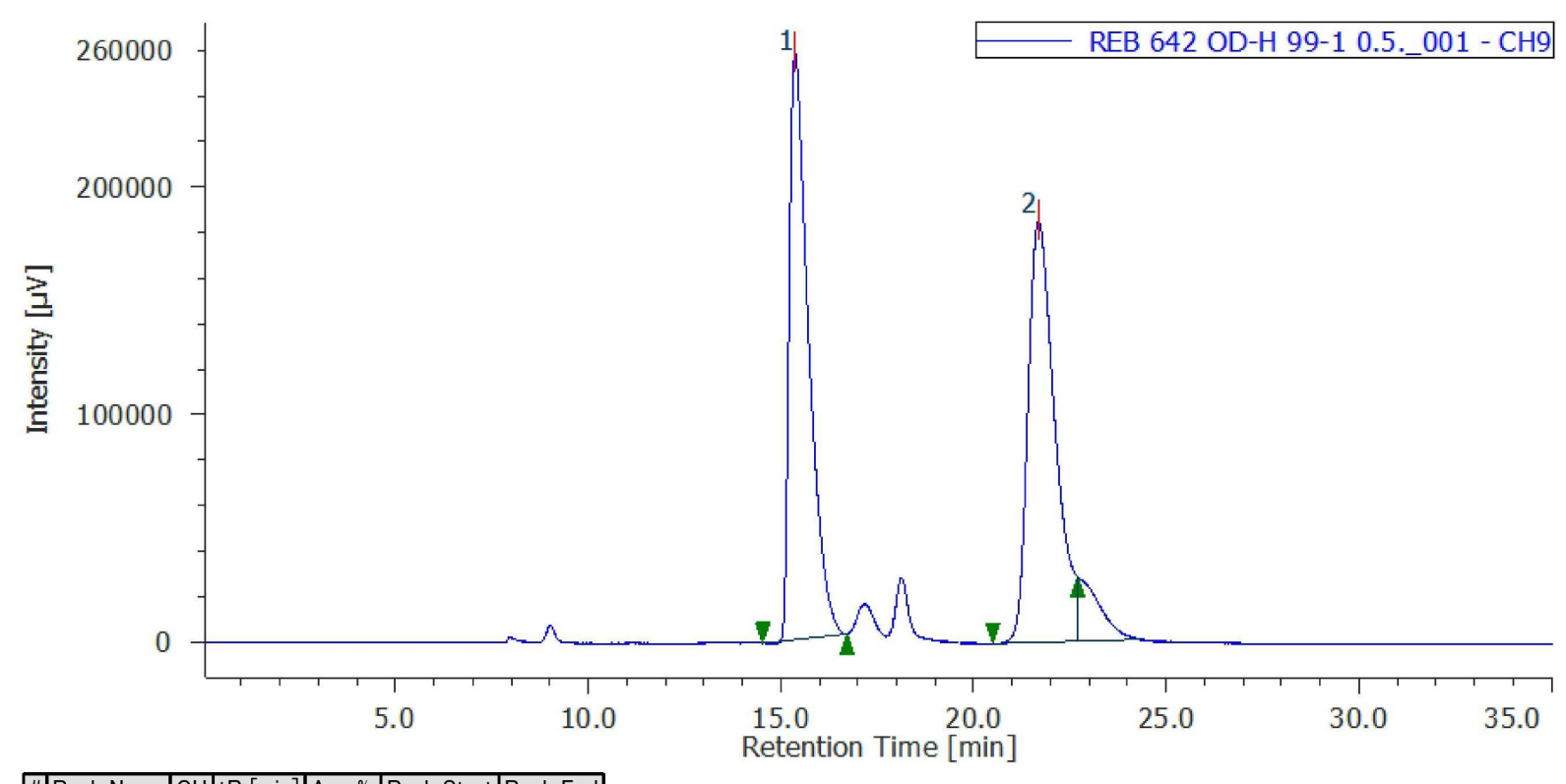

\begin{tabular}{|l|l|l|l|l|l|}
$\#$ Peak Name & CH & tR [min] & Area\% & Peak Start & Peak End \\
\hline
\end{tabular}

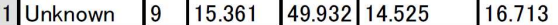

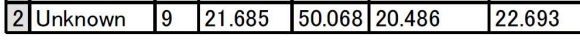

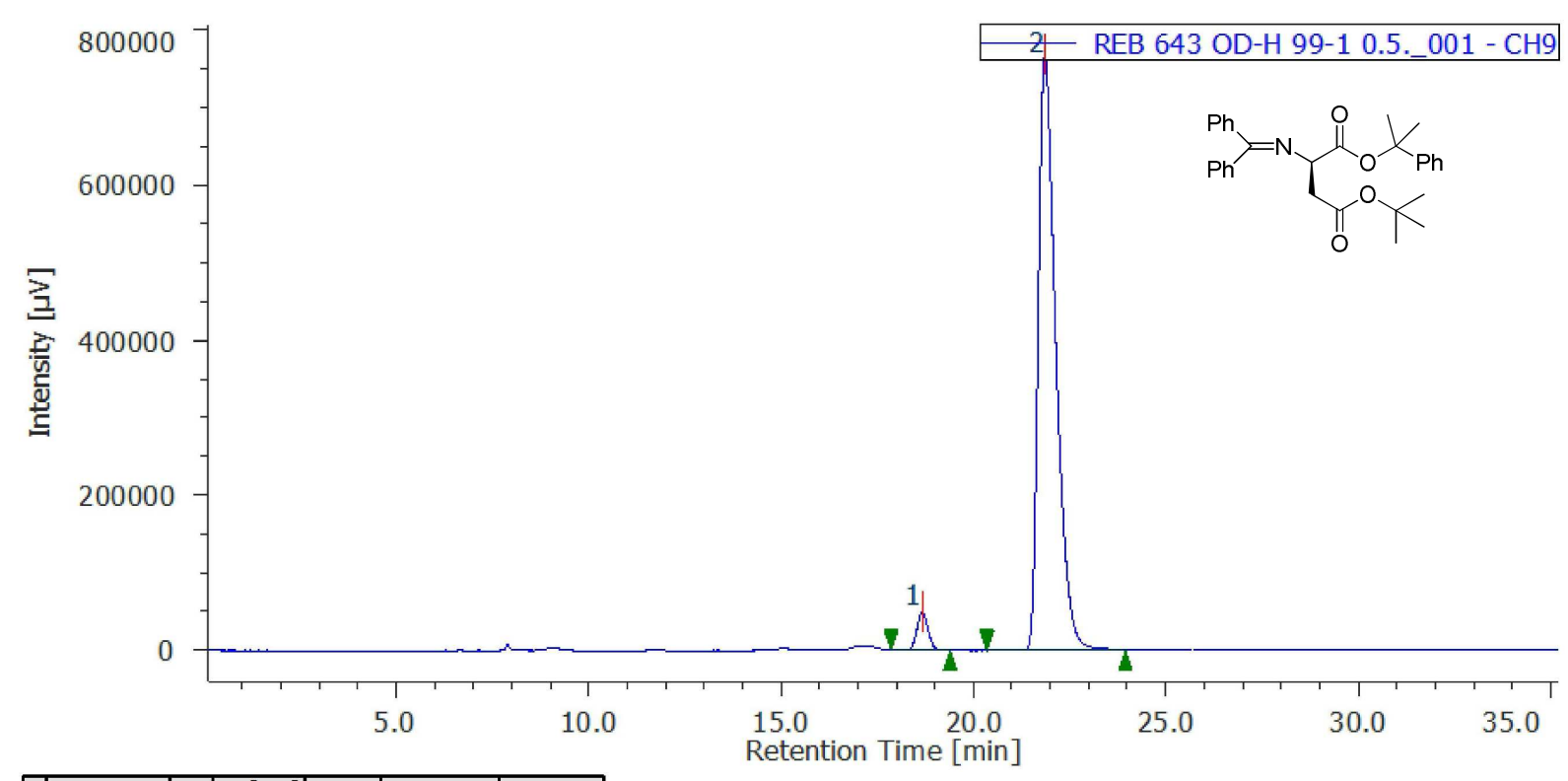

\begin{tabular}{|l|l|l|l|l|l|}
$\#$ Peak Name & CH & tR [min] & Area\% & Peak Start & Peak End \\
\hline
\end{tabular}

\begin{tabular}{|l|l|l|l|l|l|l|}
\hline 1 & Unknown & 9 & 18.660 & 3.997 & 17.858 & 19.390 \\
\hline
\end{tabular}

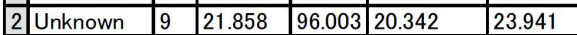

